Review

\title{
New perspectives in imaging of uveal melanoma
}

\author{
Malgorzata Solnik ${ }^{1, \sharp}$, Natalia Paduszynska ${ }^{1,4}$, Anna M. Czarneckaa ${ }^{2,3}$, Kamil J. Synoradzki ${ }^{4}$, Yacoub A. Yousef ${ }^{5,}$ To- \\ masz Choragiewicz ${ }^{6}$, Robert Rejdak ${ }^{6}$, Mario Damiano Toro ${ }^{7,8,}$ Katarzyna Dyndor $^{9}$ and Michal Fiedorowicz ${ }^{4, *}$
}

1 Faculty of Medicine, Medical University of Warsaw, 02-091 Warsaw, Poland; m.solnik98@gmail.com (M.S.), s073975@student.wum.edu.pl (N.P.)

2 Department of Experimental Pharmacology, Mossakowski Medical Research Institute, Polish Academy of Sciences, 5 Pawinskiego Str., 02-106 Warsaw, Poland; anna.czarnecka@gmail.com (A.M.C.)

3 Department of Soft Tissue/Bone Sarcoma and Melanoma, Maria Sklodowska-Curie National Research Institute of Oncology, 5 Roentgen Str.,02-781 Warsaw, Poland; am.czarnecka@pib-nio.pl (A.M.C.)

4 Small Animal Magnetic Resonance Imaging Laboratory, Mossakowski Medical Research Institute, Polish Academy of Sciences, 5 Pawinskiego Str., 02-106 Warsaw, Poland; mfiedorowicz@imdik.pan.pl (M.F.), ksynoradzki@imdik.pan.pl (K.J.S.)

5 Department of Pediatric Surgery, Ministry of National Guard Health Affairs, King Abdulaziz Medical CityJeddah (KAMC), King Saud Bin Abdulaziz University for Health Sciences, COM-J, King Abdullah International Medical Research Center, Riyadh 11481, 1515 Jeddah, Saudi Arabia; yamyousef@gmail.com (Y.A.Y)

6 Department of General Ophthalmology and Pediatric Ophthalmology Service, Medical University of Lublin, Lublin, Poland; rrejdak@yahoo.com (R.R)

7 Department of General Ophthalmology, Medical University of Lublin, 1 Chmielna Str., 20-079 Lublin, Poland. toro.mario@email.it (M.D.T.)

8 Eye Clinic, University of Catania, Via S. Sofia 78, 95123 Catania, Italy

9 Departament of Radiography, Medical University of Lublin, 8 Jaczewskiego Str., 20-090 Lublin, Poland; dyndorka@interia.pl (K.D.)

\# These authors contributed equally

* Correspondence: mfiedorowicz@imdik.pan.pl

Simple Summary: Uveal melanoma is the most prevalent intraocular tumor in adults, derived from melanocytes, and the liver is the most common site of metastases. Due to troublesome tumor localization, different imaging techniques are utilized in diagnostics, i.e., ultrasonography (US), optical coherence tomography (OCT), single-photon emission computed tomography (SPECT), positron emission tomography/computed tomography (PET/CT), magnetic resonance imaging (MRI), fundus fluorescein angiography (FFA), indocyanine green angiography (ICGA), fundus autofluorescence (FAF).Specialists eagerly use these techniques, but sometimes precision and quality of obtained images are imperfect, which raises diagnostic doubts, which prompts the search for new ones. In this review, in addition to analyzing the currently utilized methods, we also introduce experimental techniques that may be adapted to clinical practice in the future. Moreover, we raise the topic and present a perspective for personalized medicine in uveal melanoma treatment.

Abstract: Uveal melanoma is the most common primary intraocular malignancy in adults characterized by insidious onset and poor prognosis strongly associated with tumor size and the presence of distant metastases, most commonly in the liver. Contrary to most tumor identification, biopsy followed by pathological exam is not recommended in ophthalmic oncology. Therefore, early and non-invasive diagnosis is essential to enhance patients' chances for early treatment possibilities. We reviewed imaging modalities currently used in the diagnosis of uveal melanoma, i.e., fundus imaging, ultrasonography (US), optical coherence tomography (OCT), single-photon emission computed tomography (SPECT), positron emission tomography/computed tomography (PET/CT), magnetic resonance imaging (MRI), fundus fluorescein angiography (FFA), indocyanine green angiography (ICGA), fundus autofluorescence (FAF). The principle of imaging techniques was briefly explained, along with their role in the diagnostic process and a summary of their advantages and limitations. Further, the experimental data and the advancements in imaging modalities were searched. We described their innovations, showed current usage and research, and explained the possibilities of utilizing them to diagnose uveal melanoma and their potential application in personalized medicine such as theranostics.

Keywords: uveal melanoma; diagnosis; imaging; MRI; PET; CT; SPECT; OCT; ultrasonography 


\section{Introduction}

The uveal melanoma is a neoplastic lesion that originates from melanocytes [1]. Although the disease is relatively uncommon, it is the most prevalent primary intraocular malignancy in adults. The annual prevalence varies between 2 per million in southern Europe to 8 per million in northern Europe [2]. Differences may be connected with increased ocular pigment content in southern populations [1]. The average incidence among Caucasians worldwide is 5,1 per million [3]. The symptoms appear in middle age, about 60 years old, and are more common in men [4]. The tumor can occur in the anterior uveal tract that contains the iris or posterior, containing the choroid and ciliary body [5]. Among uveal melanomas, even $90 \%$ derives from the choroid, $7 \%$ from the ciliary body, and 3\% from the iris $[3,6,7]$.The main risk factors include light eye color, fair skin, and the ability to $\tan [1,8,9]$. Fair hair color $[1,8]$ and exposure to ultraviolet light other than welding were not statistically significant risk factors [10]. Established association between oculodermal melanocytosis, iris, and choroidal nevus, and uveal melanoma were found. Patients with present atypical and common cutaneous nevi and cutaneous freckles are also at risk [1].

At the time of diagnosis, clinically evident metastases are detected in $2-4 \%$ of all patients $[11,12]$. Due to hematogenous spread, the liver is the primary and the most common site of metastasis - $91 \%$ [13-15]. Other typical sites include lung (28\%), bone (18\%) and skin $(12 \%)$ [15]. It is indicated that micrometastases are present since the diagnosis because of frequent systemic recurrences and the identification of circulating tumor cells in the bloodstream of patients who do not have clinically detected metastases [16,17]. Even with successful local treatment, over $30 \%$ of UV patients will develop metastases within ten years [18].

The overall prognosis of uveal melanoma is rather poor. Once the metastatic stage is diagnosed, overall survival varies from 3 to 16 months, with a mortality rate of $92 \%$ within two years [19]. Tumor size is one of the most important prognostic factors [1,20-22]. Small tumors ( $<3 \mathrm{~mm}$ tumor thickness and $<10 \mathrm{~mm}$ basal diameter), medium tumors $(3-8 \mathrm{~mm}$ tumor thickness and $<15 \mathrm{~mm}$ basal diameter), and large tumors $(>8 \mathrm{~mm}$ tumor thickness and $>15 \mathrm{~mm}$ basal diameter) have estimated 5 -year mortality of $16 \%, 32 \%$, and $53 \%$, respectively. With every one-millimeter increase in thickness, patients have a $5 \%$ bigger risk of metastasis at ten years [22]. Older age at the time of diagnosis [23], the male gender is also associated with unfavorable prognosis [20,24]. Based on tumor location, iris melanoma has the best prognostication than ciliary body melanoma [1,22]. Genetic factors such as chromosome $3,1 \mathrm{p}$ loss, or $8 \mathrm{p}$ gain correlate with increased risk for metastasis $[1,20]$. When it comes to the histopathologic features, tumors dominated by epithelioid cells, with high mitotic activity and a large mean diameter of ten largest nucleoli, are linked to poor prognosis $[1,20,25,26]$. In the American Joint Committee on Cancer classification (AJCC), uveal melanoma is graded based on tumor basal diameter and thickness, extraocular extension, and involvement of the ciliary body. Poor prognosis was associated with the advanced AJCC stage [20]. 


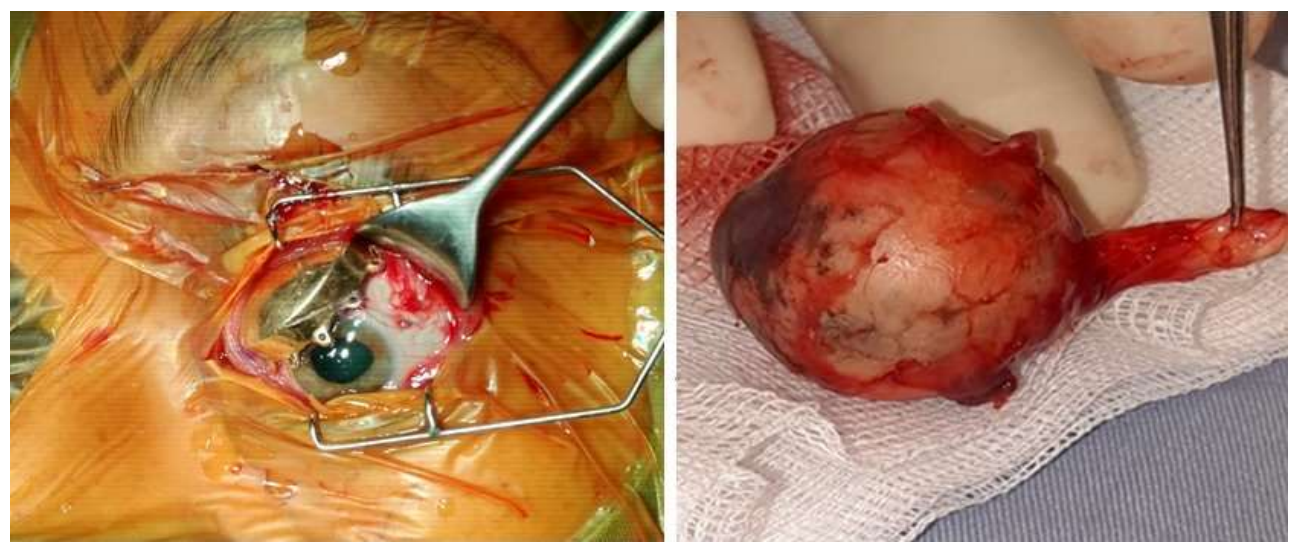

Figure 1. Main Treatment options for choroidal melanoma. Small and medium size choroidal melanoma can be treated by Radioactive Plaque therapy (A), or by proton therapy, while very large tumors generally mandate Enucleation (B).

The therapeutic methods undertaken after the diagnosis of uveal melanoma and recommended by National Comprehensive Cancer Network (NCCN) Guidelines based on radiotherapy (brachytherapy, fractionated stereotactic irradiation), particle beam radiation, and surgical treatment (Figure 1) [27]. Other techniques like laser and photodynamic therapy, cryotherapy possess occasional use $[1,18,27,28]$. Radiation therapy is most commonly used for the treatment of UM. Brachytherapy is a form of radiotherapy containing plaques with isotopes emitting gamma radiation towards the tumor $[3,29]$. During the proton beam therapy, tantalum rings are positioned at the tumor margin and constitute the border for radiation [30]. The stereotactic radiosurgery is based on radiation of gamma rays directed to circumscribed areas saving adjacent structures [31,32]. Apart from the mentioned, conservative treatment includes transpupillary thermal therapy and photodynamic therapy $[33,34]$. The extensive and advanced tumors are treated with radical methods as enucleation (a surgical extraction of the globe), exenteration (removal of an eye and ambient tissue), and uvectomy (selective removal of anterior uveal tract tumors) $[1,35,36]$. Surgical technique (enucleation) is recommended for tumors too large for brachytherapy, difficult to treat only by radiation, extensive extraocular extension, or optic nerve involvement [27]. The efficiency of chemotherapy using cytotoxic substances in hepatic metastatic lesions is insignificant [3,35]. There are also known chemo-, immuneand radioembolization, the isolated and percutaneous hepatic perfusion [3]. Several clinical trials analyze the potential possibilities of using the monoclonal antibodies as uveal melanoma immunotherapy $[37,38]$.

Table 1. The summary of advantages and limitations of currently available diagnostic techniques

\begin{tabular}{|c|c|c|c|}
\hline $\begin{array}{l}\text { Imaging } \\
\text { method }\end{array}$ & Advantages & Limitations & Resolution \\
\hline US & $\begin{array}{c}\text { non-invasive } \\
\text { wide availability } \\
\text { elementary tool in the initial diag- } \\
\text { nostic } \\
\text { visualization of lesion shape, struc- } \\
\text { ture, and vascularization } \\
\text { good imaging of retinal detach- } \\
\text { ment, vascular malformations, and } \\
\text { hemorrhage }\end{array}$ & $\begin{array}{l}\text { operator-dependent method } \\
\text { diagnostic difficulties in case of } \\
\text { muscles atypical localization or } \\
\text { vortex veins enlargement } \\
\text { conditions mimicking uveal mela- } \\
\text { noma like choroidal naevus, reti- } \\
\text { nal hamartoma, tuberculoma, } \\
\text { neurilemmoma } \\
\text { tumors less than 1mm in thick- } \\
\text { ness could be unnoticed }\end{array}$ & $\begin{array}{c}150 \text { and } 450 \mu \mathrm{m}, \\
30 \text { and } 60 \mu \mathrm{m}(\mathrm{UBM})\end{array}$ \\
\hline
\end{tabular}


good assessment of extrascleral extension and adjacent structures involvement

ultrasound biomicroscopy as a developed method in diagnostics relatively low price between $\$ 155$ and $\$ 721$ overestimation of the tumor dimension compared to MR

\begin{tabular}{|c|c|c|c|}
\hline OCT & $\begin{array}{c}\text { non-invasive } \\
\text { radiation-free } \\
\text { high spatial resolution } \\
\text { can provide images of tissue micro- } \\
\text { structure } \\
\text { can visualize small lesions in the } \\
\text { anterior eye segment } \\
\text { low price of around } \$ 200 \text { on aver- } \\
\text { age }\end{array}$ & $\begin{array}{l}\text { small tissue depth that can be vis- } \\
\text { ualized } \\
\text { not suitable for imaging of pig- } \\
\text { mented lesions as image shadow- } \\
\text { ing occur } \\
\text { limited use for detection and } \\
\text { measurements of posterior eye } \\
\text { segment tumors }\end{array}$ & $\begin{array}{c}10 \mu \mathrm{m} \text { or up to } 1 \mu \mathrm{m} \text { in } \\
\text { UHR-OCT }\end{array}$ \\
\hline FFA/ICGA/FAF & $\begin{array}{c}\text { non-invasive } \\
\text { radiation-free } \\
\text { easily accessible } \\
\text { possible differential diagnosis } \\
\text { use in treatment follow-up (FAF) or } \\
\text { assessing the proper placement of } \\
\text { radioactive plaque (FAF) }\end{array}$ & $\begin{array}{c}\text { motion artifacts } \\
\text { subjective and non-quantitative } \\
\text { interpretation of images } \\
\text { low diagnostic accuracy when } \\
\text { used alone }\end{array}$ & 7.4 to $5 \mu \mathrm{m}$ \\
\hline SPECT & $\begin{array}{l}\text { non-invasive } \\
\text { more sensitive than PET in primary } \\
\text { tumor diagnosis } \\
\text { suitable for small uveal melanoma } \\
\text { diagnosis } \\
\text { possible diagnosis in atypical mani- } \\
\text { festation or ocular complications } \\
\text { use of melanic specific radionu- } \\
\text { clides (123I-IMP) } \\
\text { less expensive (\$1900 on average) } \\
\text { and more available than PET/CT }\end{array}$ & $\begin{array}{l}\text { accurate diagnosis is highly time- } \\
\text { dependent (up to } 48 \mathrm{~h} \text { ) } \\
\text { not suitable for amelanotic mela- } \\
\text { noma diagnosis } \\
\text { exposure to radiopharmaceuticals } \\
\text { and X-ray radiation with the use } \\
\text { of SPECT and CT hybrid }\end{array}$ & $\begin{array}{l}9.3 \mathrm{~mm} \text { full width at half } \\
\text { maximum (FWHM) }\end{array}$ \\
\hline MRI & $\begin{array}{l}\text { non-invasive without exposition to } \\
\text { ionizing radiation } \\
\text { favorable soft-tissue contrast } \\
\text { a convenient method to visualize } \\
\text { intra- and extraocular involvement } \\
\text { good to measure the tumor } \\
\text { used to detect distant metastases, } \\
\text { especially in the liver } \\
\text { used in choosing the therapeutic } \\
\text { method }\end{array}$ & $\begin{array}{l}\text { difficult to distinguish amelanotic } \\
\text { melanoma from melanotic ones } \\
\text { choroidal metastases could mimic } \\
\text { a uveal melanoma } \\
\text { difficult to distinguish tumor } \\
\text { from vitreous hemorrhage } \\
\text { movement artifacts } \\
\text { relatively long duration time } \\
\text { about } 20 \text { minutes } \\
\text { the relatively high price of } € 200- \\
€ 1000\end{array}$ & $\begin{array}{l}\text { 3T MRI } 800 \mu \mathrm{m}, 7 \mathrm{~T} 500 \\
\text { to } 650 \mu \mathrm{m} \text { and } 32 \mu \mathrm{m} \\
\text { obtained by } 9.4 \mathrm{~T} \text { MRI }\end{array}$ \\
\hline
\end{tabular}


PET/CT non-invasive

diagnosis of the medium and large

primary tumor

sensitive in the diagnosis of distant metastases, especially in the liver suitable for the metastatic disease prognostication can evaluate early treatment response with higher sensitivity than MRI not widely available risk of falsely positive results in inflammation, infection, trauma not suitable for diagnosis of the small primary tumor exposure to radiation resulting in increased cancer risk very expensive (\$2227 on average)
PET cameras can provide images with a spatial resolution of around $2.4 \mathrm{~mm}$ full width at half maximum (FWHM)
CT High sensitivity, whole-body imaging, a large area covered during acquisition, moderate/high resolution, less movement artifact, scanning of a broader range of patients (with metal depositions, pacemaker), short time of scan (anxiety patients or with claustrophobia) false positives, radiation exposure, high cost of replacement $X$ ray tubes, time-consuming analysisof data
FI Noninvasive, safe, easy to perform, cost-effective, digital photos can be easily stored and transmitted for consultation inability to access deeper layers of the retina, optical aberrations or cataract influence on image quality, 2D representation of the spatial structure (possible artifacts, peripheral aberrations)
Depends on CCD or phone camera resolution

(14 $\mu$ m per pixel) 


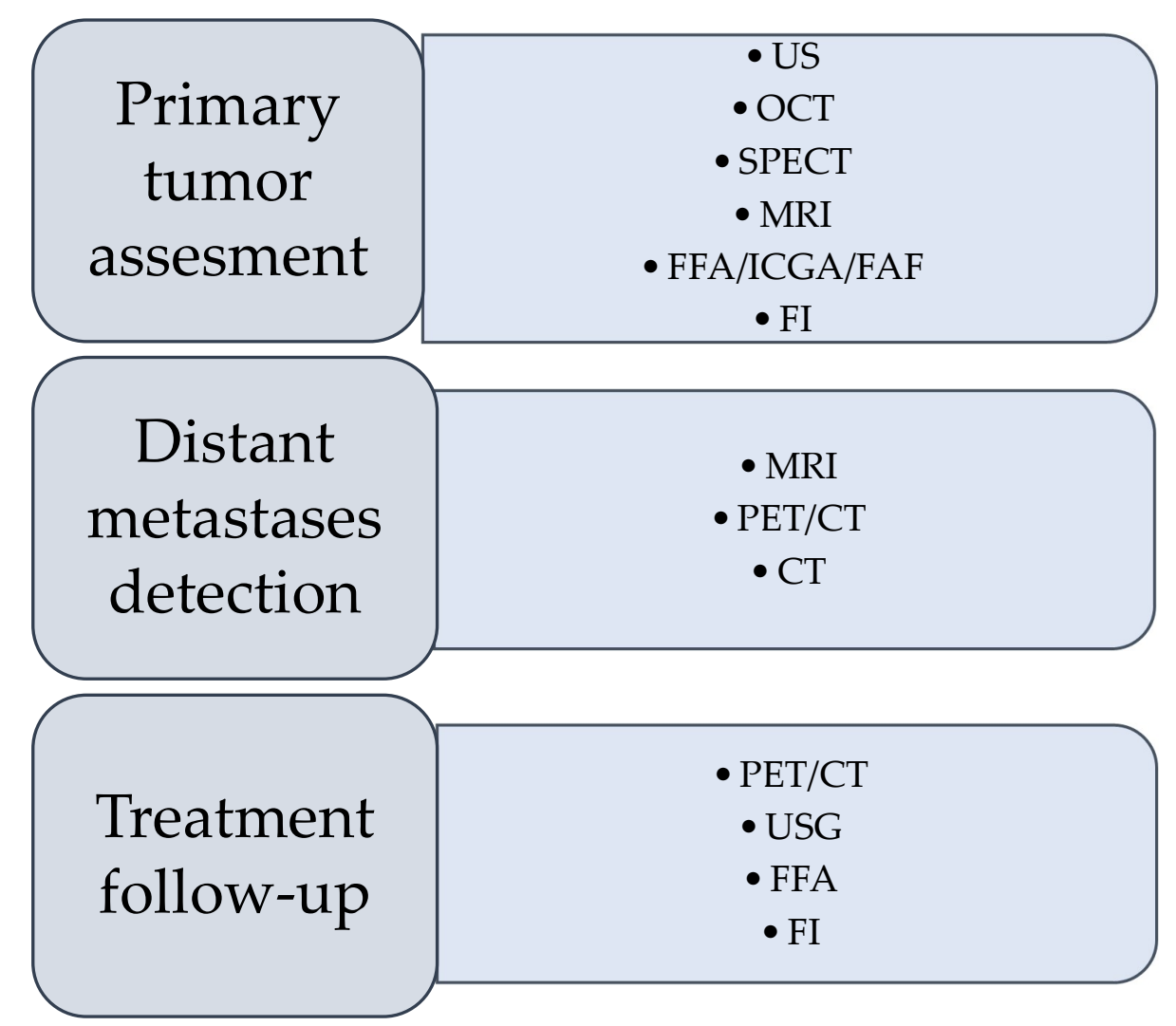

Figure 2. Preferable imaging techniques in different stages of diagnosis and follow-up of uveal melanoma. US =ultrasonography, OCT = optical coherence tomography, SPECT = single-photon emission computed tomography, MRI = magnetic resonance imaging, FFA = fundus fluorescein angiography, ICGA = indocyanine green angiography, FAF = fundus autofluorescence, PET = positron emission tomography, $\mathrm{CT}=$ computed tomography.

The gold standard of tumor confirmation is biopsy followed by histopathological examination. However, this does not apply in the diagnosis of ocular tumors, including uveal melanoma, since fine-needle aspiration biopsy could lead to the seeding of tumor cells. Additionally, non-specific clinical presentation and poor prognosis, especially after diagnosing metastatic disease, show the importance of early and non-invasive identification of uveal melanoma $[39,40]$. In this article, we analyzed the current diagnostic options for uveal melanoma, highlighted their limitations, and reviewed the recent experimental data. 

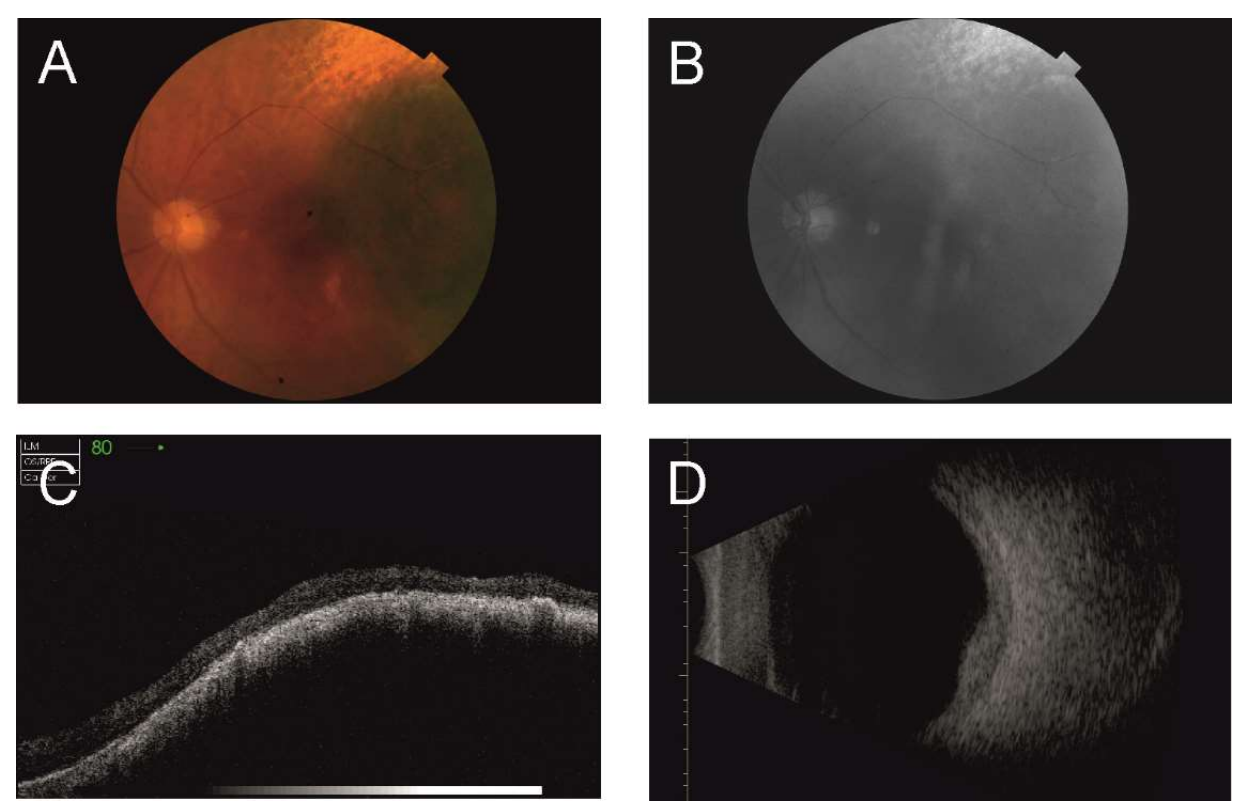

Figure 3. Choroidal melanoma: fundus photography (A), red free image (B), OCT scan (C), and US (D).
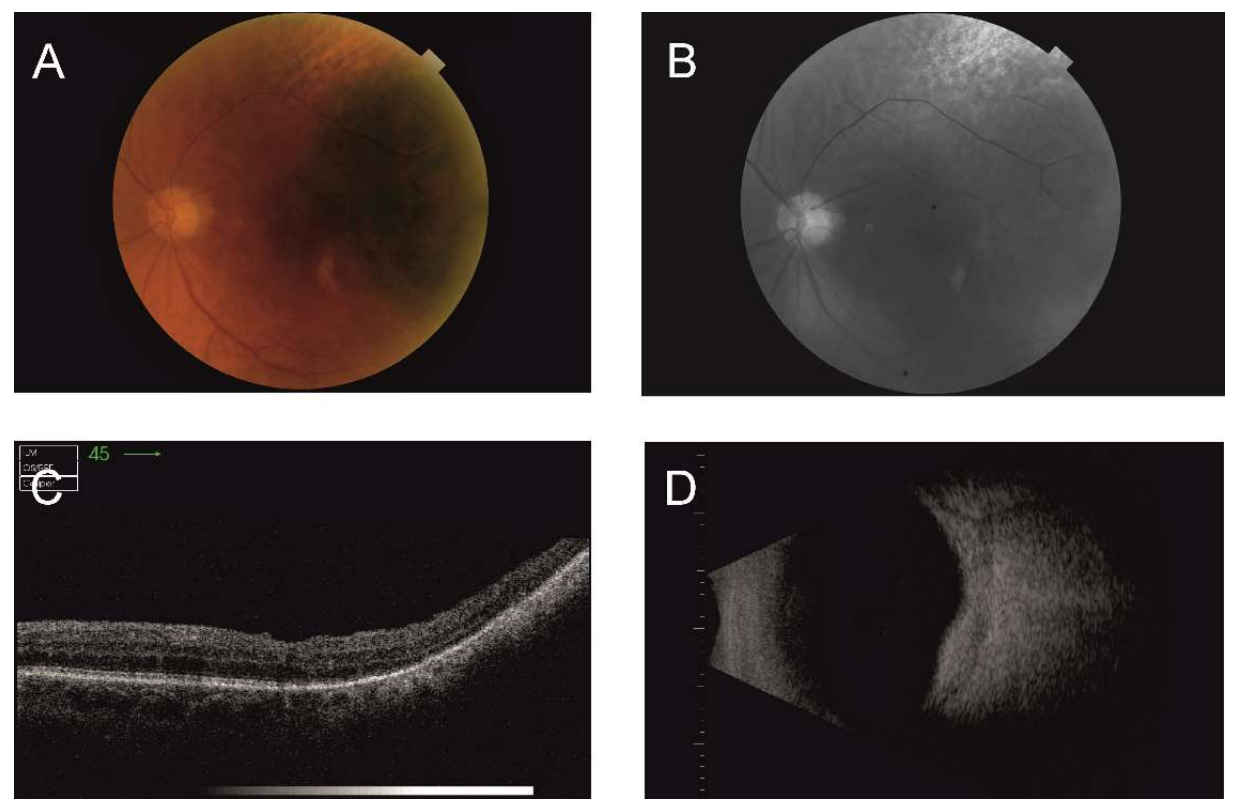

Figure 4. Choroidal melanoma imaged after brachytherapy. Reduction of the tumor volume (A, B, C) as well as increased echo density (D) are visible.

\section{Imaging techniques currently used in the diagnosis of uveal melanoma}

\subsection{Fundus imaging (FI)}

Indirect ophthalmoscopy is the primary and most important method to diagnose UM (Figure 3) [41,42].In this technique, a direct ophthalmoscopy image of the patients' retina appears directly on the observer's retina [43]. This observation allows determining several defining features like the size of the tumor, retinal detachment (in tumors greater than 4 $\mathrm{mm}$ in thickness), presence of pigmentation, its location (distance to the foveola and optic 
disc), extrascleral extension towards the anterior direction, involvement of ciliary body $[42,44]$.

Fundus photography (FP) performed initially allows assessing retinal pathology. By FP ophthalmologist may examine the central retina, macula, optic nerve, and after mydriatics, peripheral retinal area. It is a primary method of retinal imaging [45]. Choroidal melanoma looks like a dome, mushroom-shaped or diffuse form. The color of the tumor is gray to greenish brown. Retinal detachment, necrosis, or atrophy of retinal pigment epithelium cells may occur [46]. Fundus photography allows to detection of small lesions with a basal diameter lower than $3 \mathrm{~mm}$ [47].

Nowadays, wide-angle systems can capture up to $200^{\circ}$ by utilizing montage images. Wide-angle systems could observe shallow margins of tumors and are precisely like USG, but these systems are characterized by low sensitivity in detecting lesions localized anterior to the equator [46,48-50].

Imaging of fundus by photography evolve from analog film-based to digital pictures. Nowadays, images are obtained by ocular fundus cameras with digital photography. Recently, smartphone-based fundus imaging is developed (smartphone connected with digital fundus camera). Compared to conventional digital fundus photography, smartphonebased fundus imaging is a low-cost alternative to imaging retina. This equipment requires less training time for a person performing the diagnosis. Obtained images possess a good quality comparable to computers [51,52].

Due to portability, better availability, non-infrastructure requirements (continuous power supply), and obtaining by non-specialists personnel (images can be sent for further analysis for retina specialists), this technique may improve the study of more patients in the future, especially in communities with less developed medical infrastructure in primary care [53].

Photographs of the retina let to observe small lesions in diagnosing uveal melanoma but consists of a baseline. To diagnose these tumors, additional techniques like OCT or US are additionally used [54]. Alone fundus photography may be useful to monitor tumor growth, assessing therapy treatment, or arise recurrences $[46,55,56]$.

\subsection{Fundus fluorescein angiography, indocyanine green angiography, and fundus autofluorescence}

Fundus fluorescein angiography (FFA) is an imaging technique used to examine circulation in the retina and choroid and provide information about the retinal pigment's blood-retina barrier integrity and characteristics of epithelium (Figure 5). Intravenous administration of fluorescein dye (sodium fluorescein) is followed by illuminating the retina with blue light at a wavelength of $465 \mathrm{~nm}$. A photon of light is absorbed by an electron at its resting stage, causing its excitation. Further, as the electron relaxes to the resting stage, a photon on yellow-green light is released. This process is captured by a series of quickly obtained photographs or a film taken after dye injection $[57,58]$.

In large uveal melanomas, intrinsic tumor circulation is paired with choroidal circulation. This double circulation, late leakage, and hot spots resulting from pinpoint leakage from retinal pigment epithelium (RPE) are characteristic features of FFA in uveal melanomas. However, this imaging modality alone lacks diagnostic accuracy [42,55]. Meyer et al. [59] assessed the diagnostic accuracy of FFA in the diagnosis of choroidal melanoma between $17 \%$ and $75 \%$, with $58 \%$ on average. Nevertheless, bundling multiple images acquired from FFA, OCT, fundus autofluorescence could help increase the accuracy of diagnosis [60]. On the other hand, FFA is a valuable method for differential diagnosis, as it can detect hemangioma, hemorrhage, or choroidal detachment [55,61]. FFA is also used in follow-up after brachytherapy to detect complications arising from radiotherapy, such as radiation retinopathy and radiation maculopathy $[41,56]$. 

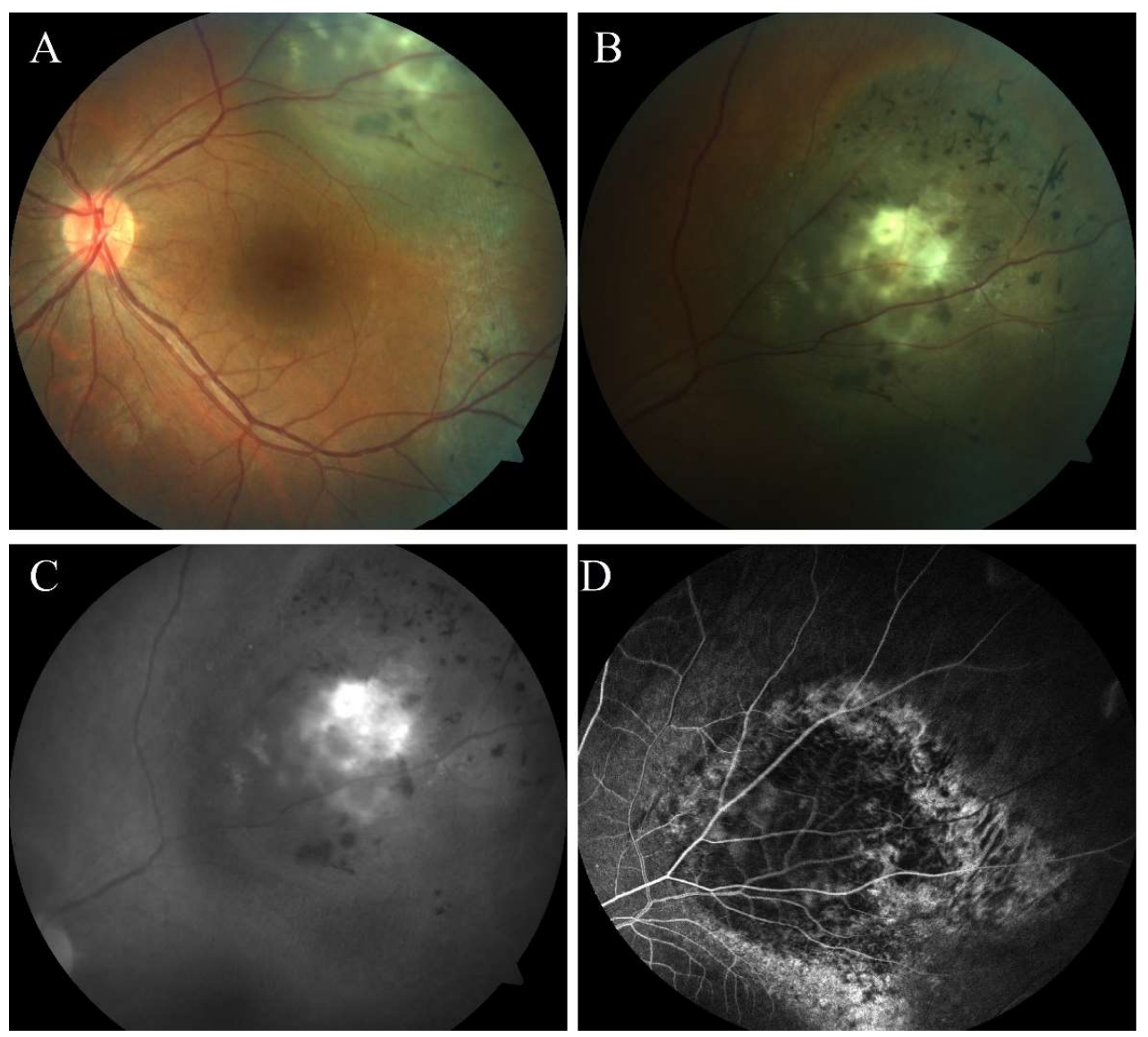

Figure 5. 30-year-old female with left melanotic choroidal melanoma (A). Clinical exam showed RPE hyperplasia and metaplasia indicating underlying previous choroidal nevus (B). The lesion has orange pigment lipofuscins that showed fundus auto fluorescence (C), and the mass was associated with intrinsic vascularity in FFA (D).

Indocyanine green angiography (ICGA) is similar to FFA, except that the fluorescein dye used in this method is indocyanine green. Its peak absorption is at around $790 \mathrm{~nm}$, with a peak emission of $835 \mathrm{~nm}$. These properties allow penetration of retinal layers, melanin, macular pigment making it possible to visualize structures beneath RPE. Indocyanine green binds to plasma protein in about $98 \%$, making it impossible for the dye to exit the bloodstream through choriocapillaris fenestrations, allowing visualization of not only large and medium vessels but choriocapillaris as well [60,62].

ICGA provides greater visualization of tumor vasculature as it can imagine microcirculation of uveal melanoma, especially when there is overlying blood [42,44]. Variable patterns of ICGA (hypofluorescent, isofluorescent, and hyperfluorescent) depend on tumor thickness and degree of pigmentation [61]. These non-specific patterns disqualify ICGA as an accurate diagnostic modality; however, ICGA can potentially differentiate pigmented and non-pigmented choroidal melanomas and choroidal hemangiomas. Nonpigmented tumors compared to pigmented tumors show earlier onset of fluorescence $(<1$ min vs. $3 \mathrm{~min}$ ), and choroidal hemangiomas reveal characteristic findings on ICGA, including early onset of fluorescence and early maximum fluorescence followed by "washout" of the dye in later frames $[55,63]$. Application antireflection coated lens and indium iodide lamp allow to obtain $7.4 \mu \mathrm{m}$ resolution [64]. It should also be mentioned that ICGA could induce phototoxicity on RPE cells, and unnecessary use should be avoided [65].

Fundus autofluorescence (FAF) is an in vivo imaging technique that allows visualization of stimulated emission of light coming from fluorescence chemical compounds (fluorophores) found in the ocular fundus with fundus camera containing specific filters [60]. 
The most common fluorophore is lipofuscin that accumulates in RPE cells and is seen as orange pigment on indirect ophthalmoscopy [66]. Secondary epitheliopathy that is common in malignant choroidal tumors causes the shedding of photoreceptor outer segments. Combined with high phagocytic activity, this process increases lipofuscin accumulation seen in uveal melanomas $[60,66]$.

One of the predictive risk factors of small melanoma is an overlying orange pigment visualized with FAF. Therefore, FAF could play a role in the early detection of small choroidal melanomas and differentiation between choroidal melanoma and nevus [67]. Choroidal melanomas demonstrate clumps of hyper-autofluorescence, whether most of the choroidal nevus is iso-autofluorescence or hypo-autofluorescence [42,44,66]. Additionally, blue light FAF can be used to confirm the proper location of radioactive plaque used in local treatment [44]. Finally, all of these fluorescence-based modalities are dependent on the patient's ability to stay still to avoid motion artifacts and experience in the interpretation of acquired images [57,60]. Fundus autofluorescence images can be obtained by confocal scanning laser ophthalmoscope with blue light excitation or fundus camera with appropriate filters. These methods allow to achieve 5 or $14 \mu \mathrm{m}$ per pixel resolution, respectively [68].

\subsection{Ultrasonography}

Ultrasonography (US) is a diagnostic tool based on sending and receiving sound waves (with a frequency greater than $20000 \mathrm{~Hz}$, inaudible to humans) with a probe (containing piezoelectric transducer) which is both a transmitter and receiver of waves (Figures 3-4, 6-8) [69]. In ophthalmology, there are two types used, A-mode scan to carry out measurements or movability assessment and B-mode scan to visualize intraocular structures of different echogenicity as a view grayscale created based on reflected waves [70]. These modes use different frequencies, the A-mode $8 \mathrm{MHz}$ and the B-mode $10 \mathrm{MHz}$ [71].

Uveal melanoma on A-scan has low to medium reflectivity and presents a positive angle kappa sign, which means that the spikes are high and decrease toward the sclera [1]. On B-scan uveal melanoma is a homogenous mass with a low acoustic profile [72]. Because the eye has high fluid content, it provides an acoustic window and facilitates relatively detailed visualization. If the tumor presents rich vascularization, the pulsations can be registered [73]. The mass can resemble a mushroom shape, and it is synonymous with Bruch's membrane disruption [54]. Ultrasound can facilitate the assessment of adjacent structures involvement because the extraocular area has a higher reflectivity than neoplasm, so infiltration is visible as regions of hyporeflectivity beyond the physiological limits of the sclera, which are marked by echoes from orbital fat $[56,74,75]$. Thus, the US is suitable for assessing the tumor extrascleral extension and visualizing lesions even 2 $\mathrm{mm}$ [74]. Moreover, it enables the determination of lesion shape and general structure [76]. The average cost of the US in the world is estimated between $\$ 155$ to $\$ 721$ [77].

The classic US allows to evaluation neoplasm of the anterior segment. Still, ultrasound biomicroscopy (UBM), which uses higher frequencies (from $35 \mathrm{MHz}$ to $50 \mathrm{MHz}$ ), facilitates the visualization of lesions derived from the ciliary body, tumor progression to posterior regions to the iris, and differentiation of cystic or solid nature of the lesion [55]. UBM with application of a higher frequency compare to B-scanners, allow to obtain better axial and lateral resolution 30 and $60 \mu \mathrm{m}$ (150 and $450 \mu \mathrm{m}$ in B-scanner) [78]. Due to increased resolution in UBM, it is possible to reveal the posterior margin of the tumor and evaluate the disease extension [79]. The disadvantage is the relatively low availability of that method. 

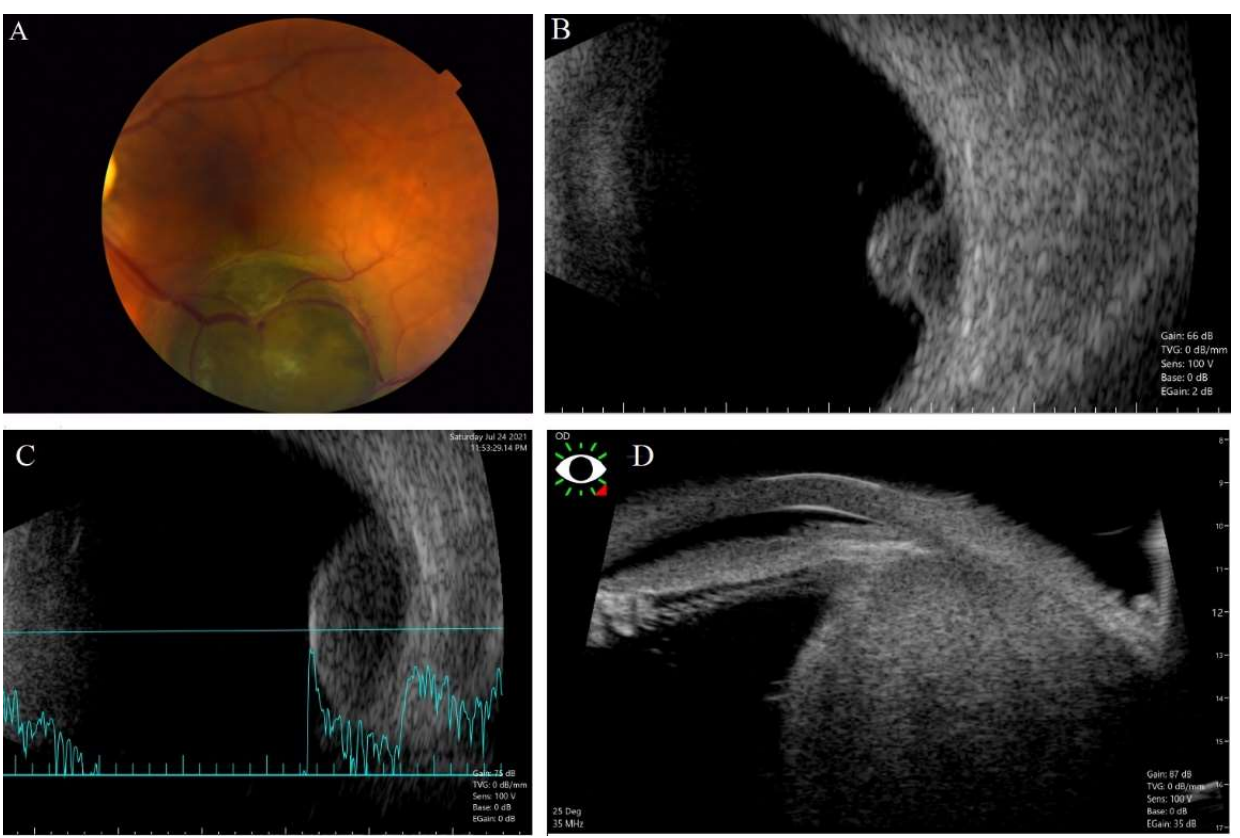

Figure 6. Echographic features of choroidal melanoma. (A) Melanotic choroidal melanoma that is mushroom shape as detected in B scan (B) and shows acoustic hollowness. (C) Another patient with dome shape choroidal lesion associated with moderate low internal reflectivity in B-scan and A-scan suggestive of choroidal melanoma. (D) Patient with ciliary body melanoma detected in ultrasound bio microscopy (UBM).

Scott et al. [80] also compared the effectiveness of US, magnetic resonance imaging(MRI), and computed tomography (CT) and revealed 100\% sensitivity for an ultrasound. Martin et al. [81] suggested that the US should be an elementary tool in the initial diagnosis of choroidal melanoma and control during the treatment. Because of high ultrasound penetration compared to light through the ocular tissues, B-mode imaging is considered a more effective diagnostic process tumor than anterior-segment optical coherence Tomography (AS-OCT)[1]. The retinal detachment, a concomitant condition with uveal melanoma, could be successfully detected by the US because of the accumulation of subretinal fluid which splits the layers (choroid and retina), which generally are seen as integrated and that also enables the visualization of choroidal growth located behind the damaged retina [82]. Other conditions apart from tumors or retinal detachment as hemorrhage and vascular malformations, can be successfully detected with US [83].

The diagnostic process of the neoplastic lesion could be complicated when it is located close to the optic nerve if the ocular muscles are atypically situated or in the case of vortex veins enlargement $[75,81]$. The ocular disorders which sonographic appearance is similar to melanoma are choroidal nevus, retinal hamartoma, tuberculoma, or neurilemmoma [55]. The tumor thickness is a relevant limitation of the B-scan method because lesions $<1 \mathrm{~mm}$ could be unnoticed [54]. The size measurements are performed with a high frequency of about $10 \mathrm{kHz}$ probe [84]. The significant limitation is that the higher frequency used, the higher the image resolution, the greater wave attenuation, and the reduced penetration depth. Due to the accuracy and precision of the method, the tumor sizes could be overestimated by the 2D US compared to MR with the average difference value of $1 \mathrm{~mm}$ [84]. In research studies, three-dimensional US facilitates the analysis of extent scale, but it is not performed in clinical practice [85]. 

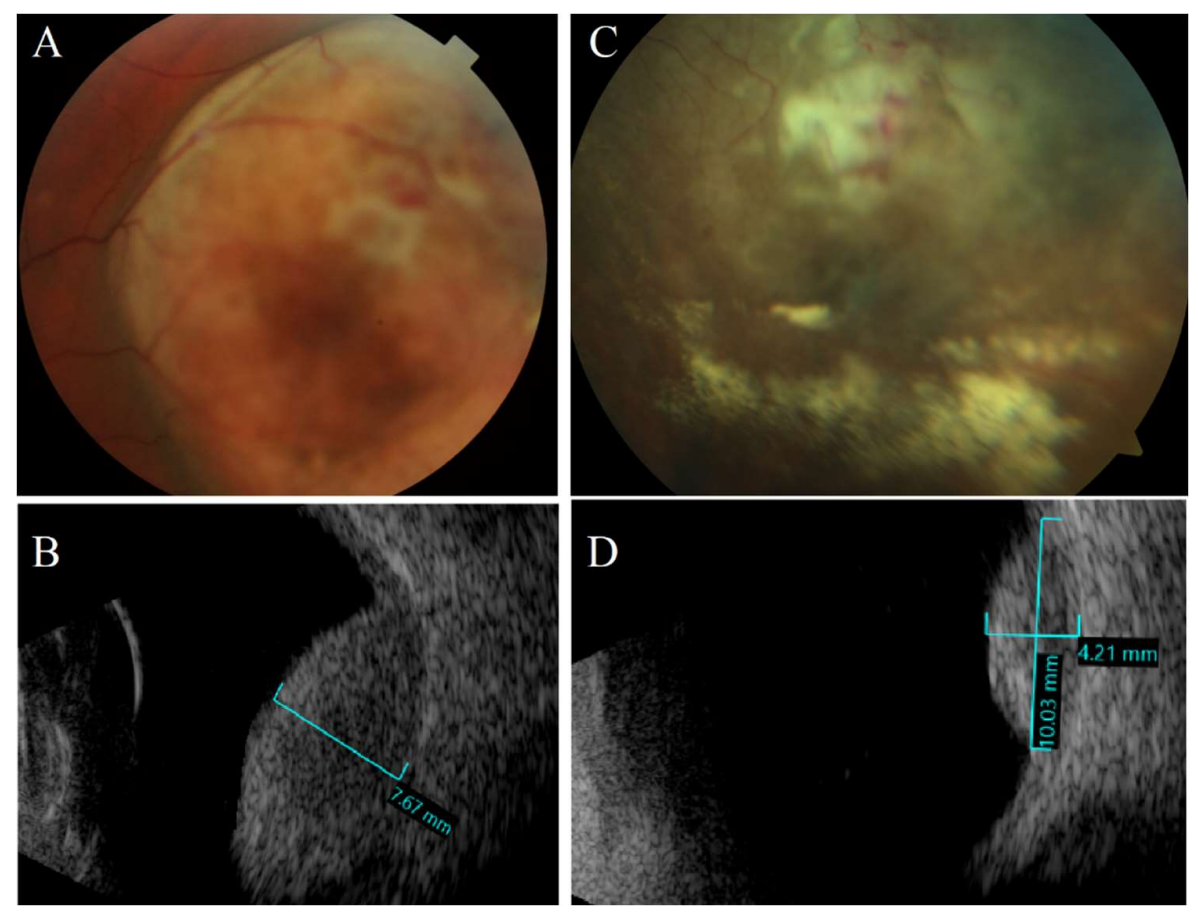

Figure 7. This left eye had amelantic choroidal melanoma (A) and B-scan showed $7.7 \mathrm{~mm}$ thick dome shape tumor with moderate low internal reflectivity. 6 months after treatment by I-125 Radioactive plaque, the tumor showed regression clinically (C), and thickness decreased to $4.2 \mathrm{~mm}(\mathrm{D})$.

Color Doppler (CDI) is a non-invasive method that shows blood flow in vessels considering flow direction. Doppler function (CDUS) could serve as a tool to evaluate the response to radiation treatment because the untreated lesions characterize richer vascular patterns than untreated ones [55]. Although ultrasound is widely available, it is operatordependent method, and only two-dimensional images can be obtained while other methods allow making reconstructions [84]. The other disadvantage is no penetration of tissues like bones and poor imaging of tissues around the perimeter of the wave beam [86].

\subsection{Optical coherence tomography}

Optical coherence tomography (OCT) is a diagnostic method analogous to the US, which essence, is receiving and processing light waves (with a near-infrared wavelength) to create cross-sectional images (Figures 3-4) [87]. It uses the interferometry technique; the light is split into a reference beam and sample beam and then reassembled. Echo time delay and reflected or backscattered light intensity are being measured to provide images of tissue microstructure. With depths of 1-2.5mminto the tissue, OCT can provide images with a spatial resolution of $10 \mu \mathrm{m}$ or up to $1 \mu \mathrm{m}$ in Ultra-High Resolution OCT (UHROCT), the most advanced form of OCT used currently only for academic and research purposes [88]. With a low cost of around $\$ 200$, this method is also safe as no adverse events occur and no radiation is used [89]. 

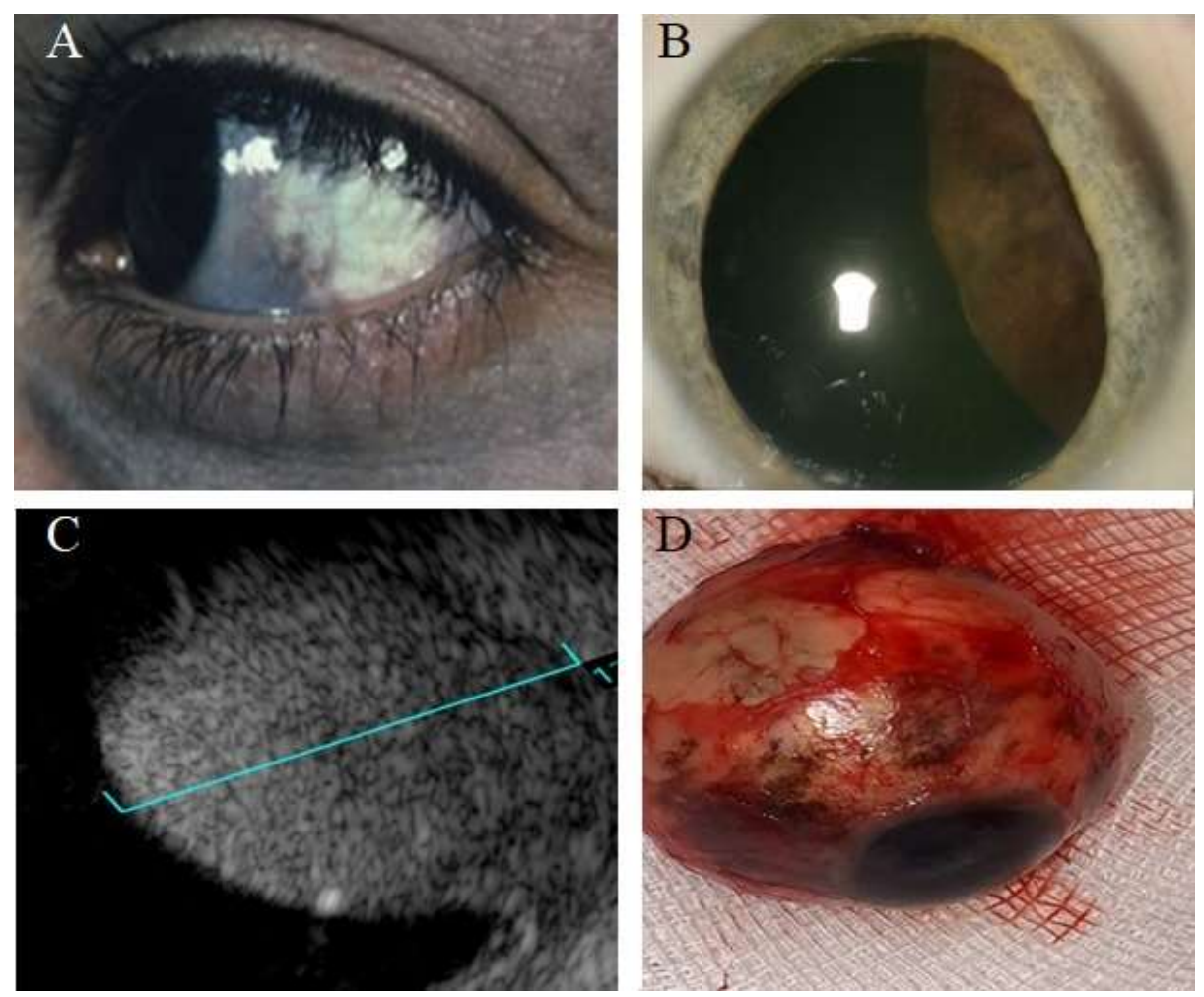

Figure 8. Patient with oculo-dermal melanosis (A) was found to have retrolenticular melanotic mass (B), which was found to be large choroidal melanoma (13 $\mathrm{mm}$ thick) with ciliary body involvement (C). This eye was enucleated and shown to have scleral melanosis (D).

The two main technologies used to create an OCT image are the time-domain (TDOCT) and the Fourier domain (FD-OCT), also known as the frequency domain. The TDOCT was developed first, and its main limitation came from the need to move the reference mirror with increasing speed to measure the light echoes coming in sequence. Later developed, FD-OCT has a reference arm with a static mirror. It allows fast scanning as well as improved image quality. Spectral-domain OCT (SD-OCT) evolved from FD-OCT significantly increased acquisition speed up to 100-fold time than TD-OCT, which in consequence can reduce motion artifacts and enhance spatial resolution [90].

The OCT is a non-invasive and non-contact method used in the diagnostic process, choosing therapeutic decisions and treatment control [56]. AS-OCT allows real-time imaging of anterior ocular segment elements such as the cornea, anterior chamber angle, sclera, iris, and lens. When it comes to pigmented tumor detection, iris melanoma, iris nevi, and iris melanocytoma can be detected by this method [55]. The conjunctival melanoma is visible on OCT as a hyperreflective structure covered with hyperreflective epithelium regardless of whether the lesion is melanocytic or not [91]. AS-OCT is a convenient technique to differentiate conjunctival melanoma from the other types of tumor and ocular surface squamous neoplasia [92]. OCT can also efficiently differentiate melanoma and melanocytoma (retinal compound and special grow pattern) [93]. According to studies, OCT could serve as a tool for differentiation conditions such as choroidal metastasis, choroidal osteoma, hemangioma, and retinal tumors [94].

Optical coherence tomography angiography (OCTA) is a new non-invasive imaging technique with the ability for both, structural and angiographic analysis in vivo, without the use of dye injection [95-98]. Recently, Neroev et al. [99] have shown the role of OCTA may be used in complex diagnosis of early UM and circumscribed choroidal hemangioma for detection of tumor's own vessels and the nature of their branching, as well as for vessel caliber comparison. Additionally, an increase in the frequency of detection of tumor`s own 
vessels will make possible early differential diagnostics of a malignant or benign tumor and may help in establishing an adequate conserving therapy. In OCT angiography, the choroidal vascular flow rate in choroidal melanoma was significantly lower than that in choroidal nevus. Ghassemi et al. [100] found a decreased flow rate of the surface microvasculature (SMV) of choroidal melanoma cases when compared with nevi. In their study, Valverde-Megías et al., have shown that eyes with choroidal nevus demonstrate similar central macular thickness (CMT), foveal avascular zone (FAZ) area and capillary vascular density (CVD) when compared to the controlateral eye. In contrast, eyes with melanoma show increased CMT, enlarged FAZ, and reduced CVD, particularly related to increasing tumor thickness. [101].

According to some authors [102-104] OCTA measurements can provide quantitative biomarkers for early detection of radiation retinopathy (RR) and/or radiation optic neuropathy, following brachytherapy in patients with UM. OCTA provided a quantitative measurement of retinal capillary changes associated with ischemia that correlated with visual acuity and radiation dose and may predict future development of radiation-induced retinal toxicity or as a quantitative endpoint to address visual prognosis. Therefore, Matet et al. [105] have shown as visual acuity of eyes with radiation maculopathy is influenced by structural and microvascular factors identified with OCTA, including FAZ area and DCP integrity.

Jing Yan Yang et al. [106] have investigated macular microvascular characteristics imaged by optical coherence tomography angiography (OCTA) in uveal melanoma (UM). Patients were treated with Conbercept injections precede by plaque radiotherapy. According to the results showed by the authors, OCTA may provide a quantitative evaluation of early retinal microvascular changes following radiotherapy. Despite its recent progress, image artifacts on OCTA are commonly encountered and appear to be more frequent in eyes with pathology and poor visual acuity, so limiting its use. Recognition of these artifacts might help improve image interpretation and decision making [95].

The important limitation is image shadowing occurring in the case of lesions that contain pigment [79]. The light penetration is limited by the sclera or iris epithelium, and AS-OCT is preferable for lesions located superficially and without pigmentation. In other cases, ultrasound biomicroscopy could be a more beneficial choice [107]. However, in a study by Torres et al. [94], all the lesions thicker than $1 \mathrm{~mm}$, which was not detected by the US, were revealed using Enhanced Depth Imaging (EDI-OCT) technique. Pavlin et al. [108] described AS-OCT as a valuable tool for detecting small hypopigmented iris tumors. However, with pigmented iris tumors, OCT visualized only the surface of lesions; their posterior margin was not detected, making thickness measurement impossible. The same happened with large hypopigmented iris tumors as their image faded out with deeper layers of the lesion. Irido-ciliary tumors could not be thoroughly examined with AS-OCT as with provided image. One could only assume the presence of a tumor, but no details can be discerned.

\subsection{Magnetic resonance imaging}

Magnetic resonance imaging involves using the magnetic properties of atomic nuclei with odd protons or neutrons numbers, most frequently protons [109].

The images obtained by this method depend on the imaging modality, e.g., PDweighted (PDw, proton density-weighted), T1-weighted (T1w, longitudinal relaxation time-weighted), T2-weighted (T2w, transverse relaxation time-weighted), or diffusionweighted imaging [110,111]. This method is also highly suitable in experimental settings (Figure 9), e.g., for studying animal models of uveal melanoma, allowing qualitative evaluation of the images and quantitative measurements of the ocular dimensions [112]. The value of the static magnetic field generated by the devices in clinical practice is typically 1.5 or 3 Tesla, but in experimental settings, the values may exceed 7 Tesla [39].The greater the magnetic field used, the more profitable the signal-to-noise ratio and the shorter acquisition time [110,113].MRI was shown to provide a more accurate measurement of the 
tumor dimensions than US; in some cases, MR diagnosis would allow eye-preserving therapy [114].

Good soft tissue contrast creates a favorable condition to visualize intra- and extraorbital compounds, tumor localization to assess tumor dimension, and the involvement of adjacent tissues $[115,116]$. Nowadays, the acquired images influence therapeutic decisions, evaluating tumor advancement, prognosis, and response to treatment [117]. The irrefutable advantage of MR is that patient is not exposed to ionizing radiation and avoids its potential complications. GrechFonk et al. estimated the average cost of MRI at $€ 200$ $€ 1000$ (the price is lower in Asia, Australia, Europe, and higher in the USA; Table 1, Figure 1). They suggested that, although the price is not lower than ultrasonography, it is still significantly lower than the possible costs of therapy [84].
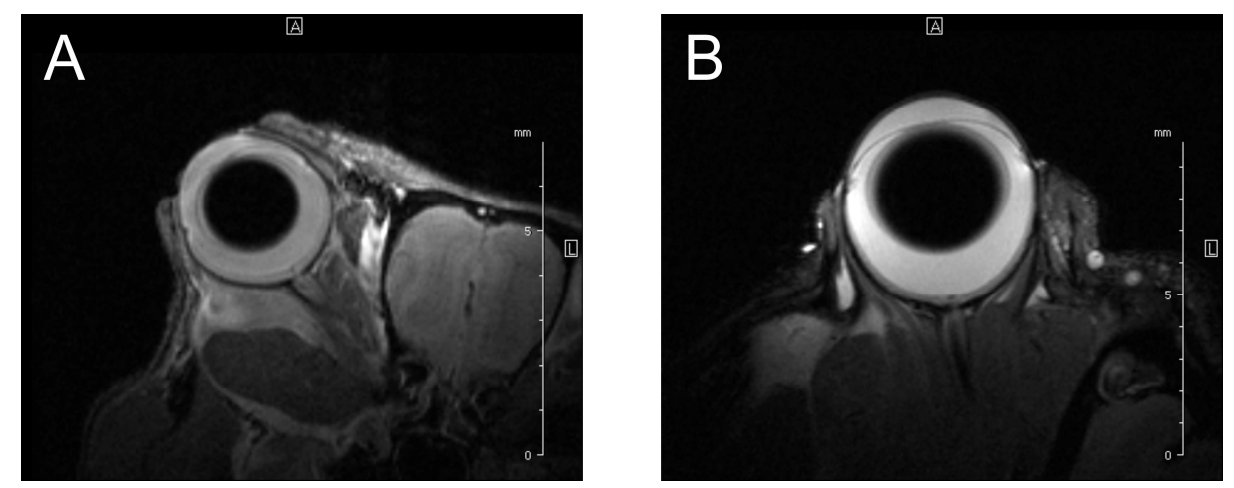

Figure 9. T2-weighted MR images of the eyes of experimental animals - (A) mouse and (B) rat. High-resolution images enable detailed evaluation of the ocular anatomy.

The presence of orbital adipose tissue enables relatively good tissue contrast [110]. The characteristic features of uveal melanoma obtained in MR (Figure 10) compared to the vitreous (because of the high-water content in the vitreous body, it is hypointense in T1w and hyperintense in T2w) include hyper-intensity on T1-weighted images, hypo-intensity on T2-weighted images, hyper-intense on PD images, and homogenous enhancement with gadolinium contrast [117-119]. Tissue contrast results from T1- and T2 - times shortening by unpaired electrons of melanin, and this property correlates with the quantity of melanin [110,120-122]. An uneven melanin distribution can contribute to inhomogeneous intensity character, similar to the disturbed vascular system of the tumor $[110,116]$. However, in the case of amelanotic melanoma, the image analysis can be troublesome because the $\mathrm{T} 2$ relaxation time will not be shortened, so the amelanotic melanoma cannot be distinguished from melanotic melanoma by this technique $[75,123]$.
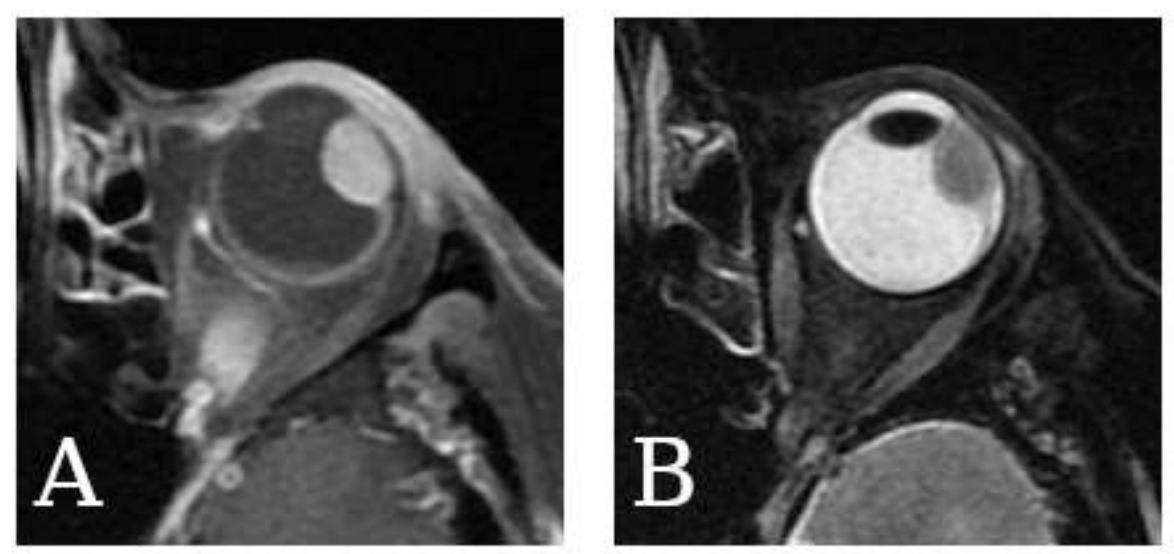
Figure 10. T1- (A) and T2-weighted (B) MR images of the human eyes with uveal melanoma.

In certain situations, the recognition of uveal melanoma may be problematic due to conditions that look similar. For example, the choroidal metastases could mimic a uveal melanoma [117]. Furthermore, the differentiation between uveal melanoma and other pathologies as vitreous hemorrhage also is challenging basing on T1w or T2w imaging [117]. Fresh blood could be isointense or hypo-intense on the T1w or T2w image, and the hyperintensity will occur after a few weeks [123]. The differences of blood in MRI appearance depend on the oxygenation of hemoglobin, oxy-, deoxy- or methemoglobin present at different stages of hemorrhage. Because of the neoplasm extension, a common concomitant condition is a retinal detachment, which could be confirmed by no contrast enhancement reaction (unlike the tumor). Still, without the use of a contrast agent, these lesions look very similar [117].

In a study by Mafee et al. [110], among 21 patients with suspected uveal melanoma and other complications as hemangioma (less contrast enhancement than melanoma), choroidal or retinal detachment, magnetic resonance imaging was an effective technique. Stroszczynski et al. [124] suggested that MR could be an even more favorable method than the computed tomography. But compared to CT, MRI is less appropriate in the visualization of calcifications [125]. According to Rescan et al., MRI is characterized by $100 \%$ sensitivity in detecting scleral involvement and extrascleral propagation with $50 \%$ and $89 \%$ specificity, respectively [126,127].3T magnetic resonance allows to acquire resolution 800 $\mu \mathrm{m}$ approx., 7T apparatus 500 to $650 \mu \mathrm{m}[84,128]$. More accurately (32 $\mu \mathrm{m})$ was obtained by high-spatial-resolution MRI at $9.4 \mathrm{~T}$ [129].

The movement artifacts resulting from involuntary eye movements are a significant obstacle in deteriorating the image quality [130]. During the procedure, drifts, tremors, saccades or blinking may occur, thus it is important to restrict the movements and one of the possible counter measure is the application retro- or parabulbar anesthesia [131]. Different devices that aim to ensure the immobilization of the globe contribute to magnetic field disturbances in the path between air and eye surface due to the permanent opening of the eyelids during the imaging process [132]. The best solution to limit artifacts would be to shorten the acquisition time, but it would negatively affect the image quality [117]. The surface coil is placed on the patient's head to improve the resolution properties but with relatively low accuracy in visualizing the deeper orbital tissues because the signal intensity decreases with the distance from the coil [117].

Outside of assessment of primary tumor, MR imaging is used to detect distant metastases, especially in the liver. Marshall et al. [133] showed that a six-monthly MRI could detect around $90 \%$ of hepatic lesions before they become symptomatic, enhancing early treatment possibilities in the group of patients with high-risk uveal melanoma. Similarly, Servois et al. [134] assessed MRI and 18F-FDG PET applicability in preoperative staging of hepatic metastases from uveal melanoma. The study showed the superiority of MRI over ${ }^{18} \mathrm{~F}-\mathrm{FDG}$ PET in this regard, with differences marked especially in lesions smaller than $10 \mathrm{~mm}$. Correspondingly, in the study conducted by Francis et al. [135], MRI presented accuracy in detecting liver lesions and determining their nature in newly diagnosed uveal melanoma patients.

Additionally, MRI serves as an important tool in the choice of therapeutic method and provides information about anatomical relationships before brachytherapy, proton beam, and stereotactic radiotherapy $[54,136]$. But considering patients with less than $6 \mathrm{~mm}$ or more than $8,5 \mathrm{~mm}$ tumor thickness, it turned out that MRI does not introduce any significant changes into their therapeutic course [84]. The duration of a single test depends on the parameters of the device and the protocol that was used.

MR imaging can also be essential in patients who underwent vitrectomy. In these cases, the replacement of the vitreous body with silicon oil (SiOil) tamponade, causes difficulties for UM diagnosis and assessing treatment follow-up with imaging methods such us US, where sound waves reflect at the SiOil-water interface [137]. SiOil by principle does 
not hinder MR imaging [137,138], although some strong artifacts may appear in the conventional protocols as the result of the off resonance of the SiOil. Dedicated MRI protocols such as $3 \mathrm{~T}$ and $7 \mathrm{~T}$ might be able to provide high-resolution images of vitrectomized eyed with SiOil tamponade [137].

The basic protocol needed to assess the tumor dimensions includes T1- and T2weighted images before and after the application of gadolinium contrast agent and lasts about 20 minutes [139]. To gain some additional and more precise information, the other sequences could be used. The GE (Gradient Echo) sequences characterize the neoplasm structure, and the SE (Spin Echo) and fat suppression sequences image the scleral boundary [117]. The multiplanar reconstructions provide measurement possibilities in various planes and reveal possible propagation beyond the sclera similar to post-contrast T1weighted scans with fat suppression, and the isotropic sequences visualize the tumor boundaries [117].The reconstructions of 3D TSE (turbo spin-echo) sequences are desirable in disturbances due to eye motion. Another modality is Diffusion-Weighted Imaging (DWI) which is based on measuring water molecules' Brownian motions depending on cellularity or the presence of edema to generate contrast on MR images. DWI may differentiate benign or malignant tumors and use ADC (Apparent Diffusion Coefficient) to analyze prognostic response to treatment by the proton beam [136,140]. A significant drawback of DWI is high susceptibility to motion artifacts. So future studies are desirable to develop a solution reducing artifacts and shortening the acquisition time.

Some other promising MR modalities include perfusion-weighted imaging with gadolinium CA clinically used in almost all oncological MR exams and has a specific UM value. Such an approach can differentiate between UM and retinal detachment [141,142]. 3D imaging may also be superior to US [143] and be used for radiotherapy planning [144]. Another remarkable approach is diffusion-weighted imaging used for differential diagnosis [140] and treatment follow-up [145].

\subsection{Computed tomography}

CT uses X-ray beams that cross the human body and are received by the detectors. Computer algorithms align obtained scans. The patient is surrounded by a ring of X-ray detectors opposite to them, localize the X-ray tube. The X-ray tube emits $\mathrm{N}$ photons per unit of time. Emitted X-ray beams passed by biological tissues attenuate a portion of radiation (this also causes a decrease in photons and energy).Detectors receive the signal and convert it into electrical pulses sent and analyzed by a computer. In the final result, data obtained by the scanning process contains cross-sectional images of the human body. Each image consists of volume picture elements called voxels. Each voxel contains information on attenuation and localization. The attenuation is measured by Hounsfield units (HU), and on the image, it corresponds to gray, but grayscale cannot be converted directly to HU [146,147].

Modern CT systems develop to several special techniques. MDCT methods (image segmentation using manual or semi-automatic approaches) allow determining the volume of objects on CT scans. It may apply in the diagnosis of osteoporosis, loss of the skeletal muscle, measuring adipose tissue, or organ volumes. CT allows localization of the lesion and its impact on adjacent tissues. Knowing of precise location facilitates planning surgical approach. CT angiography (CTA) allows assessing a vascular tree applicable in stroke evaluation, aneurysm location, size, or neurosurgery. MDCT combined with PET can highlight amyloid depositions in imaging Alzheimer's disease [146,148,149].

Computed tomography is rarely used in UM diagnosis. Other techniques as MRI or US possess a better precision and are commonly used [44]. By CT scans, it is difficult to distinguish UM from retinal detachment or and exudative macular degeneration. CT images give unambiguous results of examining epi- and extrascleral growth (e.g., in one case not lesion but macular degeneration type Junius Kulmt reveal by fundoscopy). CT enables easy distinguish vitreous from the lesion (possess high radiographic density), but small uveal melanoma lesion was impossible to detect. It is difficult to precisely determine the 
size of the tumor and the minimum measurement of a lesion. Measurements of the tumor were very inaccurate (too small, too big, or tumor was not visible) compare to the US or histological examinations [150]. In CT scanning systems for eye and orbit, approximately $1 \mathrm{~mm}$ thick slice scan, in most CT scanners spatial resolution is 500-625 $\mu \mathrm{m}$ despite the thinner than 2 mm tumors, could not be observed [149,151,152].

$\mathrm{CT}$ is nonspecific. Lesions are difficult to recognize due to the high attenuation of the signal by the choroid. MRI imaging is better than CT because melanin has intrinsic T1 and T2 shortening effects, allowing to distinguish the presence of tumor lesions [122].

CT has been used to confirm diagnostic performed previously by echography or the US to confirm the extra-scleral and extraocular extension $[80,81,153]$. It also may be useful to observe bony orbital expansion or scalloping. It also may show small calcifications [154]. CT may help in the diagnostic process in eyes with secondary vitreous hemorrhage or cataract [54].

Computed tomography may be used for accurate staging when metastasis occurs. CT and PET are used to assess liver and extra-liver (e.g., bone, lymph nodes) metastatic choroidal melanoma [155].

\subsection{Single-photon emission computed tomography}

Single-photon emission computed tomography (SPECT) uses the gamma-emitting radiopharmaceuticals delivered intravenously into patients' bodies and records their emission from many angles by the nuclear camera to provide 3D images [156]. Radionuclides used in SPECT emit one gamma-ray photon, which differentiates them from radionuclides used in positron emission tomography (PET), which emit two photons created after positron-electron encounter [157]. The nuclear cameras rotate around the patient while gamma photons radiating from the patient body pass through the collimator and finally hit the detector plane. After detectors collect all the data (location of the interaction, photon energy), they can create an image of the tissues [158]. Spatial resolution is dependent mainly on the type of used collimator as it can improve with the use of a collimator with smaller and longer holes. A $9.3 \mathrm{~mm}$ (FWHM) resolution can be achieved using all/general-purpose collimator type [156].SPECT using Technetium-99m-MIBI accumulation in tumors for evaluating ocular malignant lesions in patients allows detection of lesions in sizes 9.5 to $12 \mathrm{~mm}$ [159].

Similar to PET, gamma photons are being emitted from sites with high radionuclide accumulation. Radiotracers used in SPECT include ${ }^{99 \mathrm{~m} T c},{ }^{201} \mathrm{Tl},{ }^{67} \mathrm{Ga}$, or ${ }^{123} \mathrm{I}$. All of them have a long half-life time that varies between several hours and days, allowing a long imaging time and making the diagnostic accuracy highly time-dependent [158].

$\mathrm{N}$-isopropyl-p-[123I]iodoamphetamine (123I-IMP) is a radiopharmaceutical used initially as a tool for blood perfusion imaging. Later it was found ${ }^{123}$ I-IMP accumulates in areas with increased melanin production [160]. Goto et al. [161] found ${ }^{123}$ I-IMP SPECT is a suitable diagnostic method for uveal melanoma with atypical manifestations or for its diagnosis when ocular complications (cataract, hemorrhage, etc.) are present. In the study, a significantly high intake of ${ }^{123}$ I-IMP in an adequate tumor location was observed in 25 out of $27(92.6 \%)$ patients with suspected uveal melanoma who did not undergo any treatment. The smallest found lesion was $3 \mathrm{~mm} \times 4 \mathrm{~mm}$. In the same study, 35 out of 36 (97.2\%) patients without significant ${ }^{123}$ I-IMP accumulation were diagnosed with different intraocular disorders. In another study, ${ }^{123}$ I-IMP SPECT was done $24 \mathrm{~h}$ after the intravenous administration of radionuclide in a group of 19 patients. In all the $12(100 \%)$ patients with increased uptake of ${ }^{123}$ I-IMP, uveal melanoma was diagnosed. However, only 2 of them were SPECT positive three hours after the ${ }^{123}$ I-IMP application. In the remaining seven results, one was falsely negative $(85.7 \%)$. It was also shown that the ${ }^{123}$ I-IMP SPECT method was more sensitive and accurate than18-fluoro-2-deoxy-D-glucose (18F-FDG) PET in uveal melanoma diagnosis [162].

Abe et al. [160] evaluated the degree of usability of ${ }^{123}$ I-IMP SPECT using the uptake index (UI) and increased rations (IRs) as the defining tool. The accuracy of the 123I-IMP 
SPECT method improved with time after radionuclide administration. At $48 \mathrm{~h}$ time point and with a UI cut-off value of 1.90 , sensitivity and accuracy were $100 \%$ and $95 \%$, respectively. Moreover, calculated UI and IRs in uveal melanoma patients were significantly higher than in the group of patients without uveal melanoma. In a recent study, Kamazaki et al. [163] assessed SUV in SPECT/CT hybrid images to provide comparable UI evaluation results. Moreover, the SUV-based method evaluated at a six h time point can forecast the degree of ${ }^{123}$ I-IMP uptake at a $24 \mathrm{~h}$ time point and is more predictable than the UI evaluation method.

SPECT is superior to PET in costs, as SPECT has valued in the US \$1900 [164] on average and its availability [165]. However, due to the use of radionuclides in SPECT and even additional radiation coming from $X$-rays in SPECT/CT, increased cancer risk is a significant hazard [166].

\subsection{Positron emission tomography/computed tomography}

Positron emission tomography is a diagnostic imaging technique that visualizes areas based on their affinity and biodistribution of administered radiotracers [167]. After administering radiopharmaceutical is distributed in the tissue, it emits a positron that interacts with an electron, creating two photons emitted in virtually opposites directions. This interaction is detected and estimated within a ring of detectors placed around the patient [168]. It allows visualization as well as the quantification of said biodistribution in a three-dimensional reconstruction [167]. PET cameras can provide images with a spatial resolution of around $2.4 \mathrm{~mm}$ full width at half maximum (FWHM) [169]. The most commonly used radiotracer, ${ }^{18} \mathrm{~F}-\mathrm{FDG}$ Gallows detecting tumor cells because of their high glucose uptake [170].

Consequently, PET has been widely used in oncology to detect tumors, metastases, evaluate treatment response, and detect any recurrences due to its current availability and effectiveness [11]. However, high glucose avidity is also related to inflammation, infection, or trauma. Some treatment options, such as radiotherapy, can also cause high glucose uptake up to 3 months after the procedure is performed [171]. With the combination of computed tomography and PET, information about metabolic abnormalities is anatomically accurate, making this method much more accurate $[39,170]$.

For semiquantitative image analysis, standardized uptake volume (SUV) is used. It shows the correlation between tracer activity in tissue (microcuries per gram) and injected radiotracer dose (millicuries) as well as patient weight (kilograms). Typically, the SUV value above 2.5-3.0 indicates malignant tumors. The SUV varies based on the time between radiotracer administration and PET imaging, making it important to standardize the time interval in PET/CT scans, especially when used for treatment response evaluation [172].

For primary tumor detection, Reddy et al. [173] found 33\%of the AJCC T2 staged melanomas,75\% of T3 staged, and none of the T1 stages (with SUV cut-off value of 2.5). SUV correlated positively with the tumor volume [174]. In a different study, Matsuo et al. [175] observed high glucose uptake in nodular choroidal melanomas while diffusely infiltrating tumors were not detected. It was also shown that higher SUVmax correlated with greater thickness of the tumor. McCannel et al. [176] found correspondence between metabolic activity on PET/CT scan and monosomy 3 (54\% sensitivity, 100\% specificity). Correlation between positive PET/CT imaging and increasing tumor size along with chromosome 3 loss was also described by Papastefanou et al.[177].High SUV value was found in older patients with lesions presenting larger diameters linked to poor prognosis and greater metastatic risk [174]. According to those findings, PET/CT seems to be a useful tool for predictive purposes, but not for primary tumor diagnosis, as if it may not be able to detect small uveal melanomas or differentiate them from uveal nevi $[167,173]$.

The main role for uveal melanoma patients ${ }^{18} \mathrm{~F}-\mathrm{FDG}$ PET/CT is detecting distant metastases [170]. Both Klingenstein et al. [178] and Kurli et al. [155] reported 100\% sensitivity, $100 \%$ specificity of this method in detecting liver metastases. However, in a different 
study, Strobel et al. [179] reported PET/CT identified only 16 of 27 (59\%) known liver metastases. Freton et al. [17] described whole-body 18F-FDG PET/CT as a valuable method for initial staging in patients with UM in a retrospective study. Hepatic metastases observed on PET/CT scans were confirmed via biopsies, suggesting a $100 \%$ positive predictive value. Extrahepatic lesions (lungs, bone, lymph node, brain, spleen) also were found even though blood tests and other imaging modalities did not indicate the presence of metastatic disease in those sites. Furthermore, PET/CT seems to be valuable in assessing early therapy response. In response to the administration of chemotherapy, PET/CT showed decreased metabolic activity in lesions even though their size remained the same, according to MRI [180]. However, DW MRI can also show an early treatment response [181].

$\mathrm{PET} / \mathrm{CT}$ is an expensive imaging technique as its cost in the US varies between $\$ 429$ and $\$ 2933$ (average of $\$ 2227 ;$ Table 1, Figure 1) [182]. As well as other imaging methods using radiotracers, PET/CT scan is associated with a significant radiation dose and can increase cancer risk, so the risk-benefit ratio should be evaluated beforehand [183].

\section{New and experimental imaging techniques with the potential for diagnosing uveal melanoma}

\subsection{New radiolabeled radiotracers in PET}

As of now, ${ }^{18} \mathrm{~F}-\mathrm{FDG}$ is the most common radiotracer used in PET in oncology imaging. However, false-negative results are seen in well-differentiated tumors or lesions with low metabolic rates, and false-positive results can be observed in areas with active inflammation. Over the years, the understanding of cancer cell biology was becoming more advanced, and processes outside the glucose uptake have been discovered. Therefore, new non-FDG radiolabeled radiotracers have emerged to overcome ${ }^{18} \mathrm{~F}-\mathrm{FDG}$ limitations and better imaging capabilities for certain types of cancer [184,185].

The oldest non-FDG tracer is bone-specific sodium fluoride labeled with fluorine 18 $\left({ }^{18} \mathrm{~F}-\mathrm{NaF}\right)$. Fluorine ions bind to the surface of hydroxyapatite proportionally to the bone remodeling and bone blood flow, indicating undergoing osteolytic or osteoblastic processes. $[184,186]$.

All of this led to the use of this radiotracer in PET alone or PET/CT combination to detect and assess skeletal metastases (Table 2). Most studies were conducted on ${ }^{18} \mathrm{~F}-\mathrm{NaF}-$ PET concerning detecting bone metastases in prostate, breast, lung, and thyroid cancer [186]. In a meta-analysis, Liu et al. [187] compared ${ }^{18} \mathrm{~F}-\mathrm{NaF}$ PET/CT and ${ }^{99 \mathrm{~m} T \mathrm{Tc}-m e t h y l e n e}$ diphosphonate (MDP) planar bone scintigraphy (BS) in bone metastases diagnosis. On the patient basis, sensitivity was higher when equivocal results were described as positive (96\% vs. 93\%), but specificity was higher when equivocal results were considered as negative $(95 \%$ vs. $93 \%)$. On the lesion basis, both sensitivity and specificity showed similar results to those based on the patient basis. Compared to ${ }^{99 \mathrm{~m} T c-M D P ~ B S,}{ }^{18} \mathrm{~F}-\mathrm{NaF}$ PET/CT showed higher diagnostic accuracy in bone metastases detection with superior sensitivity and specificity. Similar results were shown in a meta-analysis by Shen et al. [188] as well.

Although this modality has not been used yet directly in the imaging of uveal melanoma, it can be applied for bone metastases detection, which is the most common site outside of liver metastases, or for assessment of extrascleral extension of the lesion.

In melanoma, available imaging methods have limited capability to detect stage I and II melanoma and small distant metastases. Consequently, new specific radiotracers that can bind to melanin have been developed, including radiolabeled antibodies, benzamide (BZA), and benzamide analogs $[189,190]$. Although presented below studies focus on cutaneous melanoma, highly specific melanin tracers should also be considered for uveal melanoma diagnosis and to detect distant metastases.

One of the more recently introduced melanin-targeted radiotracers is 5-bromo- $\mathrm{N}$ - (2-[diethylamino]ethyl) picolinamide labeled with ${ }^{18} \mathrm{~F}\left({ }^{18} \mathrm{~F}-5-\mathrm{FPN}\right)$. This benzamide derivative is characterized by a high affinity to melanin and quick renal clearance $[189,190]$. In 
the preclinical study conducted by Feng et al. [189] potential of ${ }^{18} \mathrm{~F}-5$-FPN PET in diagnosis and staging of melanoma was evaluated in vivo in B16F10 tumor-bearing mice. ${ }^{18} \mathrm{~F}-5-\mathrm{FPN}$ PET displayed high effectiveness in detecting primary tumors and simulated small metastases $(1-2 \mathrm{~mm})$ in the lungs.

Compared to ${ }^{18}$ F-FDG PET, the tumor-to-background ratio in ${ }^{18} \mathrm{~F}-5$-FPN PET was proven to be ten times higher. Nevertheless, a significant disadvantage of ${ }^{18} \mathrm{~F}-5$-FPN PET is the hepatic retention that can reduce the efficacy of this method in detecting hepatic metastases. Similar results were obtained in the study by Wang et al. [190]. Models of lymph node and pulmonary metastases were created in C57BL/6 mice and then evaluated with both ${ }^{18}$ F-5-FPN PET and ${ }^{18}$ F-FDG PET. On ${ }^{18} \mathrm{~F}-5$-FPN PET scans, small metastases in both lymph nodes and lungs were more visible, and the tumor-to background ratio was significantly higher than ${ }^{18} \mathrm{~F}-\mathrm{FDG}$ PET.

Evaluation of ${ }^{18} \mathrm{~F}-6$-fluoro- $\mathrm{N}$-[2-(diethylamino)ethyl] pyridine-3-carboxamide $\left({ }^{18} \mathrm{~F}-\right.$ MEL050), melanin-specific benzamide analog for the imaging of primary and metastatic melanoma was conducted by Denoyer et al. [191]. In that study, an ${ }^{18} \mathrm{~F}-\mathrm{MEL} 050$ PET scan was performed on melanoma model - C57BL/6 mice inoculated with cell lines of B16-F0 and B16-BL6 murine melanotic melanoma. ${ }^{18} \mathrm{~F}-\mathrm{MEL} 050$ showed a clear delineation of melanoma lesions with rapid background washout and higher tumor-to-background than ${ }^{18} \mathrm{~F}-$ FDG. Rizzo-Padoin et al. [192] confirmed this data and reported that ${ }^{18} \mathrm{~F}-\mathrm{MEL} 050$ PET/CT could detect sub-millimeter pulmonary metastases not found on ${ }^{18} \mathrm{~F}-\mathrm{FDG}$ PET/CT. Additionally, Denoyer et al. [193] reported that lymph node metastases could be identified with perilesional ${ }^{18}$ F-MEL050 administration.

To overcome significant hepatic retention of ${ }^{18} \mathrm{~F}-5-\mathrm{FPN}$, its modification ${ }^{18} \mathrm{~F}-\mathrm{N}-(2$-diethylaminoethyl)-4-(2-[2-(2-fluoroethoxy)ethoxy]ethoxy)pyridine $\left({ }^{18} \mathrm{~F}-\mathrm{PEG} 3-\mathrm{FPN}\right)$ was recently introduced. ${ }^{18}$ F-PEG3-FPN was proved to have lower liver uptake than ${ }^{18} \mathrm{~F}-5$-FPN while still demonstrating a high affinity to melanin. Hepatic and pulmonary metastases in the mouse model were detected with ${ }^{18}$ F-PEG3-FPN PET with a high tumor-to-background ratio, suggesting this imaging method for diagnosing primary and metastatic malignant melanoma [194]. Clinical application of ${ }^{18} \mathrm{~F}-\mathrm{PEG} 3-\mathrm{FPN}$ PET/MR and PET/CT is evaluated in patients with clinically suspected or confirmed melanoma in the recruiting clinical trial with a study completion date estimated at the end of 2023 [NCT04747561]. Other radiolabeled melanin-specific tracers assessed in studies include ${ }^{18} \mathrm{~F}-$ labeled picolinamide [NCT03033485] and a new experimental drug ${ }^{18} \mathrm{~F}-\mathrm{SKI}-248380$, a dasatinib-derivative [NCT01916135]. With the potential shown in cutaneous melanoma cases, studies should be expanded to uveal melanoma patients.

Table 2.Radiopharmaceuticals for PET imaging with potential for use in uveal melanoma diagnosis.

\begin{tabular}{ccccc}
\hline Feature & 18F-NaF & 18F-5-FPN & 18F-MEL050 & 18F-PEG3-FPN \\
\hline $\begin{array}{c}\text { Molecular } \\
\text { target }\end{array}$ & Bone remodeling & Melanin & Melanin & Melanin \\
\hline Current use & $\begin{array}{c}\text { Detection of } \\
\text { bone metastases } \\
\text { in prostate, } \\
\text { breast, lung, thy- } \\
\text { roid cancer, di- } \\
\text { agnosis of fi- } \\
\text { brous dysplasia }\end{array}$ & $\begin{array}{c}\text { Preclinical studies } \\
\text { - detecting small } \\
\text { metastases from } \\
\text { malignant mela- } \\
\text { noma in lymph } \\
\text { nodes and lungs }\end{array}$ & $\begin{array}{c}\text { Preclinical studies } \\
\text { - detecting small } \\
\text { metastases from } \\
\text { malignant mela- } \\
\text { noma in lymph } \\
\text { nodes and lungs }\end{array}$ & $\begin{array}{c}\text { Preclinical studies } \\
\text { metastases from } \\
\text { malignant mela- } \\
\text { noma in lungs and } \\
\text { liver }\end{array}$ \\
\hline
\end{tabular}




\begin{tabular}{|c|c|c|c|c|}
\hline $\begin{array}{c}\text { Potential use } \\
\text { in uveal mela- } \\
\text { noma }\end{array}$ & $\begin{array}{c}\text { Detection of } \\
\text { bone metastases, } \\
\text { assessing extras- } \\
\text { cleral extension } \\
\text { of the primary } \\
\text { tumor }\end{array}$ & $\begin{array}{c}\text { Detecting submil- } \\
\text { limeter metastases } \\
\text { in lungs, lymph } \\
\text { nodes }\end{array}$ & $\begin{array}{c}\text { Detecting submil- } \\
\text { limeter metastases } \\
\text { in lungs, lymph } \\
\text { nodes }\end{array}$ & $\begin{array}{l}\text { Detecting submilli- } \\
\text { meter metastases } \\
\text { in liver, lungs }\end{array}$ \\
\hline
\end{tabular}

\subsection{PET/MRI}

Discussions about combining PET and MR imaging started in the 1990s alongside the proposal of PET/CT. Great soft tissue contrast, a low dose of radiation, various imaging sequences, and possible simultaneous image acquisition suggested its promising role in oncological, neurological, and cardiovascular applications [195].

PET/MRI showed superiority over PET/CT to screen for liver metastases, especially for detecting lesions smaller than $1 \mathrm{~cm}$ [196]. Donati et al. [197] compared liver metastases detection in PET/CT, gadoxetic-acid-enhanced MRI, and retrospectively fused images of PET and MR in 37 patients. The sensitivity was significantly higher for PET/MRI (93\%) compared to PET/CT (76\%), however in gatoxetic-acid-enhanced MRI (91\%), sensitivity was proven to be not significantly different than PET/MRI. In the study conducted on 70 patients by Beiderwellen et al. [198], PET/MRI showed better diagnostic confidence and higher lesion conspicuity than PET/CT regarding the depiction of liver lesions in integrated whole-body PET/MRI. Additionally, Reiner et al. [199] proved higher accuracy of PET/MRI compared to contrast-enhanced PET/CT, but only in subcentimeter lesions without abnormal FDG uptake.

We have found no studies on PET/MRI applicability in ophthalmic oncology. However, great soft-tissue contrast and functional MRI sequences combined with a functional element of PET can provide accurate tumor information at the time of diagnosis and in therapy response evaluation in regions with complex anatomy such as ocular space [167]. Low radiation dose is another advantage, and it suggests using this modality while searching for liver metastases or in follow-up imaging. However, some challenges need to be addressed, including high costs, long acquisition time. Moreover, the research proving PET/MRI superiority over other imaging modalities was studied on only small patient groups suggesting the need for more research [195].

\subsection{Contrast agents for $M R I$}

The aim of contrast agents (CA) application in magnetic resonance imaging (MRI) and magnetic resonance angiography (MRA) is to improve the tissue contrast and visualize signal differences between contrast-containing and non-contrast-containing tissues.CA can be divided into positive which shortens T1 relaxation time and increase the signal on T1-w images, and negative which shortens T2 relaxation time and decrease the signal on $\mathrm{T} 2-\mathrm{w}$ images. The other classification distinguishes paramagnetic agents (metal ion with unpaired electrons: ${ }^{24} \mathrm{Cr}^{3+},{ }^{25} \mathrm{Mn}^{2+},{ }^{26} \mathrm{Fe}^{3+},{ }^{29} \mathrm{Cu}^{2+},{ }^{63} \mathrm{Eu}^{3+},{ }^{64} \mathrm{Gd}^{3+},{ }^{66} \mathrm{Dy}^{3+}$ and chelating ligand) and super-paramagnetic agents (iron oxide or Fe/Mn compound and polymer) [200].

Commonly used CA contain gadolinium (III) compounds, have paramagnetic properties, shorten the T1 relaxation time of water protons, i.e., they act as positive agents [201]. Gadolinium is a lanthanide that is used in the form of chelates bounded to ligands. To increase the sensitivity, special molecules are combined with gadolinium as liposomes, micelles, apoferritin, lipoproteins [200].

As previously mentioned, besides $\mathrm{Gd}^{3+}$, the other metal ions could be exploited as CA. Regarding $\mathrm{Mn}^{2+}$, both chelates (dipyridoxyl diphosphate Mn-DPDP) and manganese nanoparticle scans are used. After dissociation of DPDP, free Mn is absorbed by liver cells (intracellular agent) and excreted by the bile and DPDP ligand (which lowers toxicity) by the kidneys [202]. This compound is dedicated to the hepatic cells,although it is also taken 
up by other tissues (containing a large number of mitochondria) [202]. Pathological lesions composed of liver cells (e.g., HCC, FNH, adenoma) could be visualized on T1-w [203]. Because $\mathrm{Mn}^{2+}$ gets inside the neuron by voltage-gated calcium canal, it can act as a brainimaging tool [204]. One of the manganese compounds, Tesla scan/ mangafodipirtrisodium, has been used in clinical practice but has been withdrawn. The other magnetic ion is dysprosium $\mathrm{Dy}^{3+}$ which has a high magnetic moment [205]. It undergoes an encapsulation process and is incorporated into the liposomes, thus serving as a tool of cell surface structures (e.g., transporters) imaging [200]. Furthermore, dysprosium-based CA could enable myocardial and brain perfusion visualization [205]. None of the compounds containing dysprosium have been registered for use.

The super-paramagnetic magnetic nanoparticles (SPMNPs) are the second group of CA, which generally influences T2 relaxation time [206]. Within the MNP class, we can distinguish superparamagnetic iron oxide (SPIO) and ultrasmall superparamagnetic iron oxide (USPIO). SPIO molecules are more than 40nm in diameter, USPIO is smaller in dimension (less than $40 \mathrm{~nm}$ )and gives higher contrast on T2 images than SPIO [207]. Intravenous administration of that CA results intheir phagocytosis by macrophages and further signal reduction of the T2 signal from tissues infiltrated by cells containing CA [208]. Therefore, considering that the accumulation of macrophages increases in inflamed and infected tissue, SPIO may indicate its localization of inflammation and enable visualization of transplanted stem cells [209].

The purpose of other modifications of iron oxide nanoparticles is to target the molecules at those receptors confirmed to be present on tumor cells Yang et al. [210] proposed on the mouse model combination with of urokinase-type plasminogen activator (u-PA) and called it ATF-IO (amino-terminal fragment conjugated-iron oxide). ATF-IO binds specifically to the receptor $\mathrm{u}-\mathrm{PAR}$, which is abundant on changed cells. The paramagnetic nanoparticles directed against Robo4 and $\alpha v \beta_{3}$-integrin - markers associated with angiogenesis, were studied in imaging of melanoma in mice [211]. Indeed, they have not yet been analyzed in any research of uveal melanoma, but this makes it possible to test their usefulness in other types of cancer in the future.

Due to special coatings ( $\mathrm{SiO} 2, \mathrm{Al} 2 \mathrm{O} 3, \mathrm{Fe} 2 \mathrm{O} 3)$, the metal-alloy nanoparticles are not toxic than metallic CA [212]. Among others, this group includes Co, Fe, FeCo, and FePt [200]. The last of the mentioned compound is also called superparamagnetic iron platinum particles (SIPP). Taylor et al. [213] suggested that because more convenient relaxation features than conventional CA give the possibility to smaller cancer imaging at lower CA concentration. Encapsulation of SIPP with phospholipids into micelles and binding to the PSM antibody provides the prospect of prostate neoplasm diagnosis [213].

Magnetosomes (MS) arenano-sized crystals that are surrounded by a membrane and synthesized by Gram-negative bacteria (Magnetotactic Bacteria MTB) [214]. Those compounds could contain magnetite $\mathrm{Fe}_{3} \mathrm{O}_{4}$, greigite $\mathrm{Fe}_{3} \mathrm{~S}_{4}$ and are located inside the cell [215]. They are promising experimental tools as carriers for various substances as drugs, chemotherapeutics, or antibodies [216]. Due to bacterial movement ability provided by flagella and appropriate labeling of these organisms, it is possible to monitor their activity and the efficiency of delivery, especially that magnetosomes have an influence on T1 and T2 relaxation time (greater impact on T2 time; thus, they are negative CA) [217]. Boucher et al. [218] used magnetosomes with biogenic iron oxide and RGD peptide to visualize the contrast distribution in mice. They noticed that CA deposition in brain tumor cells occurred after 24 hours after administration. Zhang et al. [219] utilized the method to equip magnetosomes (by protein MamC) with antibodies against HER2 in the diagnostic process of breast cancer on an animal model. This method allows for selective targeting of CA to precisely defined neoplastic cells and will certainly be the research subject in the future. However, imaging is significantly more difficult when the receptors on the surface of tumor cells are not known. Xiang et al. [220] enriched magnetosomes with the P75 protein that binds selectively to MDA-MB468, SKBR3 cells (on HER2 and EGFR positive cells), and xenograft tumors. 
CA specifically targeting uveal melanoma has not been developed so far, but attempts are underway to use them differently. One of the studies aims to diagnose Crohn's disease with a new oral contrast agent (MRI) and comparison to CT imaging [NCT00587210]. The efficiency of MR lymphangiography after administration of ferumoxtran-10 (USPIO) was analyzed in the detection of the neoplasm in pelvic lymph nodes [NCT00147238][221]. Gadoxetate disodium in MRI was compared to CT in imaging of hepatocellular carcinoma and cirrhosis [NCT01341132]. In the other research, the efficiency of MRI with Gadofosveset and MR elastographyare investigated in the diagnostics of prostate cancer [NCT01761812].

\subsection{Hyperpolarized MRI}

The hyperpolarized MR imaging (HP MRI) is an innovative imaging technique of nuclear spin polarization that gives information about metabolic processes in particular tissues (including tumors) in vivo in real-time with using unique injected substrates, usually $1-{ }^{13} \mathrm{C}$ pyruvate, which participates in different metabolic pathways and converts into isotope-labeled products [222,223]. Apart from ${ }^{13} \mathrm{C}$, the other nuclei may also be hyperpolarized and serve as markers of different molecules, e.g., ${ }^{1} \mathrm{H},{ }^{29} \mathrm{Si},{ }^{15} \mathrm{~N},{ }^{89} \mathrm{Yi},{ }^{129} \mathrm{Xe},{ }^{31} \mathrm{P}[224-$ 226]. Every nucleus to be hyperpolarized must have spin-1/2 [227]. The high polarization of specific probes is obtained by external nuclear polarization and provides more enhanced signal than conventional MRI [227]. The methods to reach the effect of hyperpolarization of the molecules include, e.g., the dynamic nuclear polarization (DNP) - when spin polarization is transferred from electrons to nuclei using microwave radiation (the Overhauser effect).

Substances used in HP MRI should meet several conditions to be used as markers. The applicability of the molecule is determined by its essential role in a metabolic pathway, small dimension, ease of passing to a different cellular compartment, long T1 relaxation time, and the isotope $\mathrm{C}^{13}$ is the most common marker used in hyperpolarized probes [227].Appropriate distribution of the injected hyperpolarized substance and sufficiently long half-life time of hyperpolarized magnetization are challenging aspects of that method that require future research. The lifetime of $\mathrm{C}^{13}$-labeled markers is in the order of several tens of seconds [228].

The pyruvate is a significant component of cellular metabolism. As a product of glycolysis, it could participate in aerobic reactions (pyruvate may convert into acetyl CoA in the process of decarboxylation in which also carbon dioxide is formed, $\mathrm{CO}_{2}$ then transform into bicarbonate) and in anaerobic reactions (when pyruvate is converted into lactate in the process of dehydrogenation by LDH-lactate dehydrogenase) [229]. The characteristic feature of neoplasms associated with pyruvate transformation is the Warburg effect, in which tumor cells in aerobic conditions preferably convert pyruvate to lactate (what is called oxygen glycolysis) [230]. Visualization of the Warburg effect by HP MRI confirms the presence of neoplasm, and the assessment of that process intensity after treatment could correlate with the therapy effectiveness [231].

In addition to mentioned substances, the other markers are developed. $\left[1-{ }^{13} \mathrm{C}\right]$ acetate as a probe is dedicated to tissues whose metabolism is based on fatty acids. Acetate is converted into acetyl-CoA, which could be included in the trichloroacetic acid cycle or transform into acetylcarnitine [232]. [1-13C]alanine could serve as a precursor of pyruvate (then lactate) and facilitate monitoring that metabolite without signal interference derived from extracellular pyruvate [233]. Xiao Ji et al. suggested that alanine and glycine have more favorable parameters of level and duration of hyperpolarization, giving the possibility to long storage of the marker before application [228]. [1,4- ${ }^{13} C_{2}$ ]fumarate transformation to malate is associated with cell necrosis and positive therapy response [234]. $\left[{ }^{13} \mathrm{C}\right]$ urea (metabolically inert compound)has a dual character because it may be a probe or contrast agent [235]. [1-13 C]dehydroascorbic acid (DHA) - oxidized ascorbic acid gives information about cellular redox state (oxidative stress may coexist with cancer). It could serve as a tool to control treatment response, but the uptake of DHA varies significantly 
between different tissues, so its role as a probe is not reliable [227]. [1-13 C] succinate is a substrate of succinyl-CoA or fumarate and may give information about deregulation of oxidative phosphorylation [227]. The disorder of $\mathrm{pH}$ balance may occur in the course of cancer disease and could be detected using the following probes $\left[{ }^{13} \mathrm{C}\right]$ bicarbonate, $\left[{ }^{13} \mathrm{C} \alpha-\right.$ ketoisocaproate, $\left[{ }^{13} \mathrm{C},{ }^{15} \mathrm{~N}\right] \mathrm{N}$-(2-acetamido)-2-aminoethanesulfonic acid, [ $\left.{ }^{15} \mathrm{~N}\right]$ pyridine [227,236-238].

A significant disadvantage of this method is the high concentration of injected polarized (by DNP) substances and their potentially toxic character. In one of the clinical trials [NCT01229618], it was observed that the dose at which only mild side effects occur is 0,43 $\mathrm{ml} / \mathrm{kg}$ of HP pyruvate. The other limitation is the signal loss due to T1 relaxation and RF pulse excitation, so hyperpolarization enhances the signal only for a short time after the advertisement of the HP agent [239]. That is why the development of the techniques preventing signal losses could constitute the field of studies.

Future research should also focus on looking for more selective and targeted markers concerning different cancers. Moreover, specifically selected probes will also allow the image to detect enzymatic disruptions resulting in reaction failure and substrate accumulation typically involved in the reaction. Like conventional MR, some technical conditions need to be improved, for example, the acquisition time. The subject of further study may concern new polarization techniques (the SPINlab (automatic) polarizer with 5T magnetic field is used in patient studies [240]) or transparent differentiation of signal from normal and neoplastic tissue. Furthermore, the HP substances with relatively long half-life time should be investigated to enable more effective distribution to specific compartments. The other aspect that could be developed is receiving qualitative information about the presence of cancer and quantitative data relating the signal intensity to the stage of cancer.

Although HP MRI is not yet applicable in the diagnostics of uveal melanoma, the studies currently carried out reveal more and more new applications of that method that may be introduced into clinical practice in the future. The ongoing first phase study [NCT04772456] focuses on the safety of HP MRI and efficiency of brain tumors (gliomas) imaging after administration of $\mathrm{HP}\left[1-{ }^{13} \mathrm{C}\right]$ pyruvate in comparison to conventional MRI [241]. The visualization of lactate accumulation after injection of HP ${ }^{13} \mathrm{C}$-Pyruvate in locally advanced cervical cancer (LACC) gives a more specific metabolic characterization of the neoplasm. It is researched if co-imaging with DWI and PET would be possible to identify the areas less sensitive to radiotherapy or brachytherapy using HP MRI [NCT03129776]. Lactate levels evaluation in tumor cells is also performed in intracranial metastasis treated with stereotactic radiosurgery [NCT03324360]. There are also attempts to visualize advanced prostate cancers after administration of hyperpolarized marker (13C-pyruvate) [NCT04346225] and control the response to chemotherapy in pancreatic cancer after injection of HP pyruvate [EudraCT Number: 2016-004491-22].

\section{Personalized medicine}

Personalized medicine is promising and dynamically developing field covering both aspects of patient predispositions, diagnostics, clinical management, prognosis determination, and treatment [242]. On a more individual basis, the development of medicine will only be possible thanks to improved diagnostic methods that allow detecting specific differences influencing the choice of therapeutic decision. Genetic tests provide the most detailed and thorough information. Shields et al. [243] analyzed the cytogenetic tumor profile of 1059 patients to individualize the diagnosis. The occurrence of uveal melanoma metastases mainly was related to specific mutations (3 complete monosomy, 6disomy, $8 \mathrm{q}$ gain, $8 \mathrm{p}$ loss, and three complete monosomy, 6 disomy, and $8 \mathrm{q}$ gain). It was also noticed that the other mutations as GNAQ, GNA11, SF3B1, EIF1AX, BAP-1, and PRAME are important in assessing the prognosis [243]. 
Indeed, extending the diagnostics with genetic changes in cancer cells significantly increases the scope of information, precision, and accuracy of diagnosis and generates further challenges to effectively use the obtained data.

\subsection{Theranostics is a step towards personalized medicine}

A promising strategy that should be mentioned here is to combine non-invasive diagnostic methods with therapy to apply personalized treatment called theranostics. The new imaging methods presented previously may be utilized in that field because they allow the use of selectively targeted agents (which may be drug-coated), control their transport to destination and therapy response, and, importantly, offer the possibility of real-time imaging. However, these methods also have limitations, e.g., reaching the target tissue by the probe. Still, various solutions are being developed to ensure the probe penetration into tumor tissue, including the use of small-sized particles (nanoparticles, liposomes, micelles), coverage with appropriate particles binding to special receptors, drug release technology induced by hypoxia, temperature, or specific $\mathrm{pH}$ ranges [244].

Due to their beneficial properties, proteins, antibodies, and aptamers could be used as theranostic tools which bind to molecules that are present in excess on tumor cells or in its environment, e.g. insulin-like growth factor 1 receptor (IGF1R), folate receptor (FR), epidermal growth factor receptor (EGFR), human epidermal growth factor receptor 2 (HER2), vascular endothelial growth factor (VEGF), integrins [245]. Combining these particles with markers allows for visualization, and the conjugation with anti-cancer drugs allows for extremely precise and localized therapy. Dal Corso et al. [246] proposed smallmolecule-drug conjugates (SMDC) which contain tripeptide Arg-Gly-Asp (RGD) sequence and are ligands for $\alpha v \beta 3$ integrin (present on tumor vessels). These conjugates can transport various drugs, e.g., doxorubicin, paclitaxel, cisplatin, or camptothecin used in systemic chemotherapy, but their toxic effect on the body is significantly reduced. It is also possible to utilize fluorescent probes activated by extracellular enzymes or intracellular lysosomal transformations [247]. This method improves the image quality as the marker only accumulates in the target tissue, and background signals are eliminated. Sun et al. [248] introduced aminopeptidase N (APN)-activated theranostic prodrug (NBFMel), which is a PET probe for diagnosis and therapy. In this case, the toxic substance to cancer cells is melphalan, which acts selectively and saves normal tissues.

An attractive solution in novel theranostic particles is the practical use of a particular feature of neoplasms, which maintains a slightly acidic pH (ca. 6.8) [249]. The iron oxide nanoparticles with $\mathrm{pH}$-sensitive magnetic nanogrenades (PMNs) can self-organize under the influence of acidic reactions. The tumors of minimal size $(<3 \mathrm{~mm}$ in diameter) are detectable through the combination of MRI and fluorescence imaging. Ling et al. [249] suggested that $\mathrm{pH}$-targeted photodynamic therapy (light radiation affects the photosensitizers to produce reactive oxygen species) could be utilized in the treatment of resistant tumors. Kim et al. [250] proposed multifunctional magnetite nanoparticles (AHP@MNPs). Apart from $\mathrm{Fe}_{3} \mathrm{O}_{4}$, they consist of photosensitizer, hyaluronic acid (AHP) and are synthesized from amines. Due to the surface charge, they can be covered with different coatings. These particles enter the tumor cells through CD44 and have dual nature. They may serve as a tool of magnetic hyperthermia therapy (magnetic nanoparticles convert electromagnetic energy to heat) and photodynamic therapy.

There are also nanoparticles which activation is enzymatically catalyzed [251]. Such an activation process is possible when the enzyme concentration in the tumor environment is increased. The molecule must be adequately designed; it should confirm easy bonding with the catalyst. One of the concepts assumes that the nanoparticle releases the drug after an enzymatic decomposition of the coating structure. The particle surface is properly modified, making it susceptible to enzymatic catalysis, or the drug is placed inside the liposome and released after the carrier's destruction. Several types of enzymes might be involved in these nanoparticle decompositions, e.g., proteases, lipases, glycosidases (e.g.,cathepsin B, peroxidase, urease, glucose oxidase, $\alpha$-amylase). This technology 
is a part of targeted cancer therapy, allows for assessing enzymatic activity, and poses a challenge to understand the tumor's enzymatic arsenal further.

Kim et al. [252] suggested making use of the fact of the increased level of $\mathrm{H}_{2} \mathrm{O}_{2}$ in cancer metastases (as an adaptation to increased amounts of reactive oxygen species) for imaging and simultaneous stimulation of the anti-tumor effect of the transported drug (prodrug 7). The fluorescence induction and the substance SN-38 detachment were due to the change of boronate moiety to a hydroxyl group in 'prodrug 7 ' by $\mathrm{H}_{2} \mathrm{O}_{2}$. Thus, such an approach ensures the selectivity of the therapy and allows controlling the efficiency of the delivery of the molecules to the target site. Similar properties can be attributed to nanoparticles which functionality is dependent on tissue hypoxia. Zhou et al. [253] studied azoderivative (AzP1) irinotecan analog. This compound consists of an inhibitor of topoisomerase I and aryl-azo benzyl alcohol (azo-derivate substances undergo a reduction in conditions of low oxygen concentration and serve as hypoxia markers). The visualization of fluorescence also allows for the quantification of the released chemotherapeutic agent.

The theranostic concept is a hope for the development in the field of oncology in the context of precise diagnostics, which will enable timely treatment. The appropriate structure of modified compounds using long-known anti-cancer substances allows for a highly selective action of these substances at the tumor site while limiting the harmful effect on other tissues and persistent side effects for the patient [254]. However, once introduced into clinical practice, the high cost of these methods remains an important issue due to their cost-effectiveness, time-consuming, and advanced technology.

Several studies are currently underway in this area (admittedly in diseases other than uveal melanoma). Somatostatin-expressing neoplasms like neuroendocrine tumors, medulloblastoma, meningioma, and neuroblastoma are treated with ${ }^{90} \mathrm{Y}$-DOTATOC, and the usefulness of ${ }^{68} \mathrm{Ga}-\mathrm{DOTATOC} \mathrm{PET} / \mathrm{CT}$ in diagnosis and treatment is assessed [NCT02441088]. Additionally, circulating tumor DNA (cDNA) will be evaluated as a theranostic marker in glioblastomas [NCT03115138]. Combining radioactive particles such as ${ }^{131}$ Iodine, ${ }^{90}$ Yttrium with appropriate proteins: MIBG or DOTATOC ensures targeting drugs to tumor cells and minimizing side effects of therapy. Concurrent application of ${ }^{131} \mathrm{I}-\mathrm{MIBG}$ and ${ }^{90} \mathrm{Y}$-DOTATOC in mid-gut neuroendocrine tumors (NETs) and SPECT/CT control will be performed in a study [NCT03044977]. In turn, the study [NCT04769817] will analyze the results of patients previously treated with Lutetium 177 $\left({ }^{177} \mathrm{Lu}\right)$-PSMA with metastatic castration-resistant prostate cancer (mCRPC) and evaluation the analogy between PSMA and ( ${ }^{18}$ F-FDG) PET/CT images.

\subsection{Immunotherapy as a component of personalized medicine}

If the theranostics of uveal melanoma develops in the future, it would be worth adopting immunotherapy (Table 3). Although the development of adequately modified particles and their production is demanding and time-consuming, it will be a promising element of effective cancer therapy [255]. Thus, increasing attention is focused on using immunotherapy in uveal melanoma, even though the eye is considered an immunologically privileged structure. It is worth noting that cancer cells use many of these mechanisms, and some even mimic them, to avoid elimination by the immune system at the sites of metastases [256]. These include the expression of many particles with immunosuppressive activity against T lymphocytes or NK cells, the ability to inactivate complement, inhibit proliferation and induce apoptosis of T cells via PD-L1 (programmed death ligand1) [256]. Therefore, monoclonal antibody anti-PD1, pembrolizumab was studied in Phase 2 clinical trials to treat advanced uveal melanoma (stages IIIA, IIIB, IIIC, IV) [NCT02359851]. Pembrolizumab with entinostat (histone deacetylase), which aims to increase the effects of immunotherapy, is also being evaluated in metastatic disease [NCT02697630]. Nivolumab is another tested anti-PD 1 antibody. Combination of nivolumab and relatimab (anti-LAG3 -lymphocyte activation gene 3 is found on NK, B, activated T cells and limits their proliferation and activation [257]) is analyzed in patients with unresectable or metastatic uveal melanoma [NCT03743766]. Apart from that, the syn- 
ergistic effect of nivolumab and ipilimumab (anti-CTLA 4 antibody, cytotoxic T-lymphocyte-associated protein-4, CTLA-4) is being investigated as a first-line treatment in patients with metastases [NCT02626962].

A different approach involves targeting the drug molecule to a compound that is in excess in this tumor, i.e., c-kit, with the participation of a monoclonal antibody such as imatinib [258]. Sunitinib also acts as an inhibitor of this kinase, and the SUAVE study compares its effects to decarbazine in metastatic uveal melanoma [NCT01551459]. While in a trial [NCT01005472] with sunitinib, temozolomide was used in patients with stages III and IV of the disease. Another kinase that was tried to be inhibited was c-MET; cabozantinib was used for this purpose. The effectiveness of this drug was assessed in relation to the compounds used in chemotherapy (temozolomide and dacarbazine) [NCT01835145] in a randomized trial. However, cabozantinib did not show the expected advantage. In turn, crizotinib is currently in the second phase of clinical trials, used as adjuvant treatment in patients at high risk of relapse after therapeutic intervention [NCT02223819]. This monoclonal antibody functions as an inhibitor of anaplastic lymphoma kinase (ALK) and c-ros oncogene 1 (ROS1).

The following molecules are also in the second phase of clinical trials: a combination of cediranib and durvalumab in metastatic UM [NCT04184518], axitinib in metastatic or unresectable UM [NCT01533948], defactinib and VS-6766 in metastatic UM [NCT04720417], niraparib in UM and mutation BAP1 and with mutation BAP1 other DNA damage [NCT03207347].

Mention should also be made of Immune mobilizing monoclonal $\mathrm{T}$ cell receptors (TCRs) against cancer ImmTAC TM molecules. These particles can redirect lymphocytes against cancer cells, and interestingly, activation affects both effector and memory cells. They are bifunctional, binding to CD3 on T cells and gp100 on UM cells [259]. Currently, the efficacy and safety of IMCGP100 (belonging to the group ImmTAC TM ) in advanced UM therapy is being studied in two clinical trials [NCT03070392], [NCT02570308].

Table 3. Investigational compounds in the treatment of uveal melanoma or metastatic disease

\begin{tabular}{ccc}
\hline Evaluated treatment & Mechanism & Clinical trial \\
\hline pembrolizumab & anti PD-1 & NCT02359851 \\
\hline pembrolizumab + entinostat & $\begin{array}{c}\text { anti PD-1 + histone deacety- } \\
\text { lase }\end{array}$ & NCT02697630 \\
\hline nivolumab + relatimab & anti PD-1 + anty LAG-3 & NCT03743766 \\
\hline nivolumab + ipilimumab & anti PD-1 + anti CTLA-4 & NCT02626962 \\
\hline sunitinib & c-kit inhibitor & NCT01551459 \\
\hline sunitinib + temozolomide & $\begin{array}{c}\text { c-kit inhibitor + DNA alkyl- } \\
\text { ating agent }\end{array}$ & NCT01005472 \\
\hline cabozantinib & c-MET inhibitor & NCT01835145 \\
\hline crizotinib & ALK and ROS1 inhibitor & NCT02223819 \\
\hline cediranib + durvalumab & VEGF inhibitor + anti-PD-L1 & NCT04184518 \\
\hline axitinib & tyrosine kinase inhibitor & NCT01533948 \\
\hline defactinib + VS-6766 & $\begin{array}{c}\text { focal adhesion kinase inhibi- } \\
\text { tor + RAF/MEK Inhibitor }\end{array}$ & NCT04720417 \\
\hline niraparib & PARP1 and PARP2 enzymes & NCT03207347 \\
& inhibitor & \\
\hline IMCGP100 & gp100-specific TCR fused to \\
anti-CD3 & NCT03070392, NCT02570308 \\
\hline selumetinib & MEK inhibitor & NCT02768766 \\
\hline
\end{tabular}




\begin{tabular}{ccc}
\hline bevacizumab + teozolomide & $\begin{array}{c}\text { VEGFinhibitor+DNA alkyl- } \\
\text { ating agent }\end{array}$ & NCT01217398 \\
\hline glembatumumabvedotin & $\begin{array}{c}\text { antibody-drug conjugate tar- } \\
\text { geted to transmembrane gly- } \\
\text { coprotein NMB }\end{array}$ & NCT02363283 \\
\hline $\begin{array}{c}\text { bortezomib+paclitaxel+car- } \\
\text { boplatin }\end{array}$ & $\begin{array}{c}\text { 26S proteasome inhibitor }+ \\
\text { cytostatic alkaloid +complex } \\
\text { compound that cross-links } \\
\text { with DNA }\end{array}$ & NCT00288041 \\
\hline & $\begin{array}{l}\text { Data as of the date 23.04.2021. Clinical trials were searched on https://www.clinicaltrials.gov/ with } \\
\text { the terms "uveal melanoma" and "uveal melanoma treatment". }\end{array}$
\end{tabular}

\section{Conclusions}

Uveal melanoma is a rare intraocular malignancy with unfavorable diagnosis closely connected to tumor size, time of diagnosis, and presence of distant metastases. Tumor biopsy followed by pathological examination is not recommended in ophthalmic oncology; therefore, non-invasive imaging modalities are necessary to evaluate patients with suspected uveal melanoma [40]. US and OCT are the elementary imaging technique in the assessment of primary tumors. Both methods are cheap, widely available, and provide information about the tumor's size, shape, structure, and vascularization, especially in the anterior segment of the eye. SPECT could also be used in the initial diagnosis, although exposure to radiopharmaceuticals and the high cost of this method make it more of an additional rather than primary imaging technique [39]. Additionally, FAF/ICGA/FAF are helpful in differential diagnosis [44]. MRI, as well as PET/CT, have limited value in the diagnosis of the primary tumor; however, they are necessary for tumor staging and detection of distant metastases [39,40].

The introduction of new advancements in imaging techniques could broaden the horizons in the diagnosis of uveal melanoma. The development of new radiolabeled radiotracers in PET allows extended differential diagnosis and detection of submillimeter metastases in the liver using melanin-specific radiotracers. Multimodal imaging with PET and MRI combined offers exceptional soft-tissue contrast that could deliverprimary tumor characteristics and be used for staging and metastases identification. Visualization of specific metabolic processes is possible with HP MRI, which could allow early detection of uveal melanoma and any metastases. Similarly, new contrast agents for MRI maximize tissue contrast and can help to detect intraocular and hepatic lesions. On the other hand, new improvements in non-invasive imaging techniques can also be utilized in personalized medicine, such as theranostics. Although the applicability of those diagnostic advancements has been yet to be proven in the group of patients with uveal melanoma, their irrefutable potential needs to be tested be in future studies.

Author Contributions: Conceptualization, M.F., and A.M.C.; writing-original draft preparation, M.S. and N.S.; writing - review and editing, M.F. and A.M.C.; supervision, M.F., and A.M.C.; funding acquisition, M.F. All authors have read and agreed to the published version of the manuscript.

Funding:This study was supported by Polish National Centre for Research and Development (grant no. LIDER/39/0215/L-10/18/NCBR/2019).

Institutional Review Board Statement: Not applicable.

Informed Consent Statement: Not applicable.

Data Availability Statement: Not applicable. 


\begin{abstract}
Acknowledgments: Project carried out with the use of CePT infrastructure financed by the European Union - the European Regional Development Found in the Operational Program "Innovative Economy" for 2007-2013.

Conflicts of Interest: The authors declare no conflict of interest. The funders had no role in the design of the study; in the collection, analyses, or interpretation of data; in the writing of the manuscript, or in the decision to publish the results.
\end{abstract}

\title{
References
}

1. Kaliki, S.; Shields, C.L. Uveal melanoma: relatively rare but deadly cancer. Eye (London, England) 2017, 31, 241-257, doi:10.1038/eye.2016.275.

2. Jovanovic, P.; Mihajlovic, M.; Djordjevic-Jocic, J.; Vlajkovic, S.; Cekic, S.; Stefanovic, V. Ocular melanoma: an overview of the current status. Int J Clin Exp Pathol 2013, 6, 1230-1244.

3. Souto, E.B.; Zielinska, A.; Luis, M.; Carbone, C.; Martins-Gomes, C.; Souto, S.B.; Silva, A.M. Uveal melanoma: physiopathology and new in situ-specific therapies. Cancer Chemother Pharmacol 2019, 84, 15-32, doi:10.1007/s00280-019-03860-z.

4. Frenkel, S.; Hendler, K.; Pe'er, J. Uveal melanoma in Israel in the last two decades: characterization, treatment and prognosis. Isr Med Assoc J 2009, 11, 280-285.

5. Blum, E.S.; Yang, J.; Komatsubara, K.M.; Carvajal, R.D. Clinical Management of Uveal and Conjunctival Melanoma. Oncology (Williston Park) 2016, 30, 29-32, 34-43, 48.

6. Spagnolo, F.; Caltabiano, G.; Queirolo, P. Uveal melanoma. Cancer Treat Rev 2012, 38, 549-553, doi:10.1016/j.ctrv.2012.01.002.

7. Dogrusöz, M.; Jager, M.J.; Damato, B. Uveal Melanoma Treatment and Prognostication. Asia-Pacific journal of ophthalmology (Philadelphia, Pa.) 2017, 6, 186-196, doi:10.22608/apo.201734.

8. Weis, E.; Shah, C.P.; Lajous, M.; Shields, J.A.; Shields, C.L. The association between host susceptibility factors and uveal melanoma: a meta-analysis. Archives of ophthalmology (Chicago, III. : 1960) 2006, 124, 54-60, doi:10.1001/archopht.124.1.54.

9. Nayman, T.; Bostan, C.; Logan, P.; Burnier, M.N., Jr. Uveal Melanoma Risk Factors: A Systematic Review of Meta-Analyses. Current eye research 2017, 42, 1085-1093, doi:10.1080/02713683.2017.1297997.

10. Shah, C.P.; Weis, E.; Lajous, M.; Shields, J.A.; Shields, C.L. Intermittent and chronic ultraviolet light exposure and uveal melanoma: a meta-analysis. Ophthalmology 2005, 112, 1599-1607, doi:10.1016/j.ophtha.2005.04.020.

11. Freton, A.; Chin, K.J.; Raut, R.; Tena, L.B.; Kivelä, T.; Finger, P.T. Initial PET/CT staging for choroidal melanoma: AJCC correlation and second nonocular primaries in 333 patients. European journal of ophthalmology 2012, 22, 236-243, doi:10.5301/ejo.5000049.

12. Finger, P.T.; Kurli, M.; Reddy, S.; Tena, L.B.; Pavlick, A.C. Whole body PET/CT for initial staging of choroidal melanoma. The British journal of ophthalmology 2005, 89, 1270-1274, doi:10.1136/bjo.2005.069823.

13. Rodriguez-Vidal, C.; Fernandez-Diaz, D.; Fernandez-Marta, B.; Lago-Baameiro, N.; Pardo, M.; Silva, P.; Paniagua, L.; Blanco-Teijeiro, M.J.; Piñeiro, A.; Bande, M. Treatment of Metastatic Uveal Melanoma: Systematic Review. Cancers 2020, 12, doi:10.3390/cancers12092557.

14. Kujala, E.; Mäkitie, T.; Kivelä, T. Very long-term prognosis of patients with malignant uveal melanoma. Investigative ophthalmology \& visual science 2003, 44, 4651-4659, doi:10.1167/iovs.03-0538.

15. Diener-West, M.; Reynolds, S.M.; Agugliaro, D.J.; Caldwell, R.; Cumming, K.; Earle, J.D.; Green, D.L.; Hawkins, B.S.; Hayman, J.; Jaiyesimi, I., et al. Screening for metastasis from choroidal melanoma: the Collaborative 
Ocular Melanoma Study Group Report 23. Journal of clinical oncology : official journal of the American Society of Clinical Oncology 2004, 22, 2438-2444, doi:10.1200/jco.2004.08.194.

16. Mallone, F.; Sacchetti, M.; Lambiase, A.; Moramarco, A. Molecular Insights and Emerging Strategies for Treatment of Metastatic Uveal Melanoma. Cancers 2020, 12, doi:10.3390/cancers12102761.

17. Torres, V.; Triozzi, P.; Eng, C.; Tubbs, R.; Schoenfiled, L.; Crabb, J.W.; Saunthararajah, Y.; Singh, A.D. Circulating tumor cells in uveal melanoma. Future oncology (London, England) 2011, 7, 101-109, doi:10.2217/fon.10.143.

18. Amaro, A.; Gangemi, R.; Piaggio, F.; Angelini, G.; Barisione, G.; Ferrini, S.; Pfeffer, U. The biology of uveal melanoma. Cancer and Metastasis Reviews 2017, 36, 109-140, doi:10.1007/s10555-017-9663-3.

19. Bustamante, P.; Piquet, L.; Landreville, S.; Burnier, J.V. Uveal melanoma pathobiology: Metastasis to the liver. Seminars in cancer biology 2020, 10.1016/j.semcancer.2020.05.003, doi:10.1016/j.semcancer.2020.05.003.

20. Kaliki, S.; Shields, C.L.; Shields, J.A. Uveal melanoma: estimating prognosis. Indian journal of ophthalmology 2015, 63, 93-102, doi:10.4103/0301-4738.154367.

21. Diener-West, M.; Hawkins, B.S.; Markowitz, J.A.; Schachat, A.P. A review of mortality from choroidal melanoma. II. A meta-analysis of 5-year mortality rates following enucleation, 1966 through 1988. Archives of ophthalmology (Chicago, III. : 1960) 1992, 110, 245-250, doi:10.1001/archopht.1992.01080140101036.

22. Shields, C.L.; Furuta, M.; Thangappan, A.; Nagori, S.; Mashayekhi, A.; Lally, D.R.; Kelly, C.C.; Rudich, D.S.; Nagori, A.V.; Wakade, O.A., et al. Metastasis of uveal melanoma millimeter-by-millimeter in 8033 consecutive eyes. Archives of ophthalmology (Chicago, III. : 1960) 2009, 127, 989-998, doi:10.1001/archophthalmol.2009.208.

23. Kaliki, S.; Shields, C.L.; Mashayekhi, A.; Ganesh, A.; Furuta, M.; Shields, J.A. Influence of age on prognosis of young patients with uveal melanoma: a matched retrospective cohort study. European journal of ophthalmology 2013, 23, 208-216, doi:10.5301/ejo.5000200.

24. Rietschel, P.; Panageas, K.S.; Hanlon, C.; Patel, A.; Abramson, D.H.; Chapman, P.B. Variates of survival in metastatic uveal melanoma. Journal of clinical oncology : official journal of the American Society of Clinical Oncology 2005, 23, 8076-8080, doi:10.1200/jco.2005.02.6534.

25. Gill, H.S.; Char, D.H. Uveal melanoma prognostication: from lesion size and cell type to molecular class. Canadian journal of ophthalmology. Journal canadien d'ophtalmologie 2012, 47, 246-253, doi:10.1016/j.jcjo.2012.03.038.

26. McLean, I.W.; Sibug, M.E.; Becker, R.L.; McCurdy, J.B. Uveal melanoma: the importance of large nucleoli in predicting patient outcome--an automated image analysis study. Cancer 1997, 79, 982-988.

27. Rao, P.K.; Barker, C.; Coit, D.G.; Joseph, R.W.; Materin, M.; Rengan, R.; Sosman, J.; Thompson, J.A.; Albertini, M.R.; Boland, G., et al. NCCN Guidelines Insights: Uveal Melanoma, Version 1.2019: Featured Updates to the NCCN Guidelines. Journal of the National Comprehensive Cancer Network J Natl Compr Canc Netw 2020, 18, 120-131, doi:10.6004/jnccn.2020.0007.

28. Zewar, A.; Nawaiseh, I.; Jaradat, I.; Khzouz, J.; Alrawashdeh, K.; Abdeen, G.; Mehyar, M.; Khurma, S.; Yousef, Y.A. Management and Outcome of Uveal Melanoma in a Single Tertiary Cancer Center in Jordan. Turk patoloji dergisi 2016, 32, 186-192, doi:10.5146/tjpath.2015.01357.

29. Jaradat, I.; Zewar, A.; AINawaiseh, I.; AlRawashdeh, K.; Khurma, S.; Mehyar, M.; Abdeen, G.; Yousef, Y.A. Characteristics, management, and outcome of patients with uveal melanoma treated by lodine-125 radioactive plaque therapy in a single tertiary cancer center in Jordan. Saudi journal of ophthalmology : official journal of the Saudi Ophthalmological Society 2018, 32, 130-133, doi:10.1016/j.sjopt.2017.12.002. 
30. Damato, B.; Kacperek, A.; Errington, D.; Heimann, H. Proton beam radiotherapy of uveal melanoma. Saudi J Ophthalmol 2013, 27, 151-157, doi:10.1016/j.sjopt.2013.06.014.

31. Sikuade, M.J.; Salvi, S.; Rundle, P.A.; Errington, D.G.; Kacperek, A.; Rennie, I.G. Outcomes of treatment with stereotactic radiosurgery or proton beam therapy for choroidal melanoma. Eye (Lond) 2015, 29, 1194-1198, doi:10.1038/eye.2015.109.

32. Dunavoelgyi, R.; Dieckmann, K.; Gleiss, A.; Sacu, S.; Kircher, K.; Georgopoulos, M.; Georg, D.; Zehetmayer, M.; Poetter, R. Local tumor control, visual acuity, and survival after hypofractionated stereotactic photon radiotherapy of choroidal melanoma in 212 patients treated between 1997 and 2007. Int J Radiat Oncol Biol Phys 2011, 81, 199-205, doi:10.1016/j.ijrobp.2010.04.035.

33. Rundle, P. Treatment of posterior uveal melanoma with multi-dose photodynamic therapy. Br J Ophthalmol 2014, 98, 494-497, doi:10.1136/bjophthalmol-2013-304432.

34. Shields, C.L.; Shields, J.A.; Perez, N.; Singh, A.D.; Cater, J. Primary transpupillary thermotherapy for small choroidal melanoma in 256 consecutive cases: outcomes and limitations. Ophthalmology 2002, 109, 225234, doi:10.1016/s0161-6420(01)00902-2.

35. Chattopadhyay, C.; Kim, D.W.; Gombos, D.S.; Oba, J.; Qin, Y.; Williams, M.D.; Esmaeli, B.; Grimm, E.A.; Wargo, J.A.; Woodman, S.E., et al. Uveal melanoma: From diagnosis to treatment and the science in between. Cancer 2016, 122, 2299-2312, doi:10.1002/cncr.29727.

36. Barker, C.A.; Salama, A.K. New NCCN Guidelines for Uveal Melanoma and Treatment of Recurrent or Progressive Distant Metastatic Melanoma. Journal of the National Comprehensive Cancer Network J Natl Compr Canc Netw 2018, 16, 646-650, doi:10.6004/jnccn.2018.0042.

37. Zimmer, L.; Vaubel, J.; Mohr, P.; Hauschild, A.; Utikal, J.; Simon, J.; Garbe, C.; Herbst, R.; Enk, A.; Kämpgen, E., et al. Phase II DeCOG-study of ipilimumab in pretreated and treatment-naïve patients with metastatic uveal melanoma. PLoS One 2015, 10, e0118564, doi:10.1371/journal.pone.0118564.

38. Kummer, M.; Schuler-Thurner, B. Immunotherapy of Uveal Melanoma: Vaccination Against Cancer. Methods Mol Biol 2017, 1499, 273-278, doi:10.1007/978-1-4939-6481-9_17.

39. Neupane, R.; Gaudana, R.; Boddu, S.H.S. Imaging Techniques in the Diagnosis and Management of Ocular Tumors: Prospects and Challenges. Aaps j 2018, 20, 97, doi:10.1208/s12248-018-0259-9.

40. Li, X.; Wang, L.; Zhang, L.; Tang, F.; Wei, X. Application of Multimodal and Molecular Imaging Techniques in the Detection of Choroidal Melanomas. Frontiers in oncology 2020, 10, 617868, doi:10.3389/fonc.2020.617868.

41. Grisanti, S.; Tura, A. Uveal Melanoma. In Noncutaneous Melanoma, Scott, J.F., Gerstenblith, M.R., Eds. Codon Publications

The Authors.: Brisbane (AU), 2018; 10.15586/codon.noncutaneousmelanoma.2018.ch1.

42. Singh, P.; Singh, A. Choroidal melanoma. Oman journal of ophthalmology 2012, 5, 3-9, doi:10.4103/0974$620 \times .94718$.

43. Timberlake GT, K.M. The direct ophthalmoscope how it works and how to use it. Availabe online: https://web.media.mit.edu/ raskar/Eye/TheDirectOphthalmoscope.pdf (accessed on

44. Jager, M.J.; Shields, C.L.; Cebulla, C.M.; Abdel-Rahman, M.H.; Grossniklaus, H.E.; Stern, M.H.; Carvajal, R.D.; Belfort, R.N.; Jia, R.; Shields, J.A., et al. Uveal melanoma. Nature reviews. Disease primers 2020, 6, 24, doi:10.1038/s41572-020-0158-0.

45. Abràmoff, M.D.; Garvin, M.K.; Sonka, M. Retinal imaging and image analysis. IEEE reviews in biomedical engineering 2010, 3, 169-208, doi:10.1109/rbme.2010.2084567. 
46. Mirchia, K.; Turell, M.; Singh, A. Imaging Modalities for Uveal Melanoma. European Ophthalmic Review 2012, 06, doi:10.17925/EOR.2012.06.01.56.

47. Fallico, M.; Raciti, G.; Longo, A.; Reibaldi, M.; Bonfiglio, V.; Russo, A.; Caltabiano, R.; Gattuso, G.; Falzone, L.; Avitabile, T. Current molecular and clinical insights into uveal melanoma (Review). International journal of oncology 2021, 58, 10, doi:10.3892/ijo.2021.5190.

48. Callaway, N.F.; Mruthyunjaya, P.J.I.J.o.R.; Vitreous. Widefield imaging of retinal and choroidal tumors. 2019, 5.

49. Jain, A.; Shah, S.P.; Tsui, I.; McCannel, T.A. The value of Optos Panoramic 200MA imaging for the monitoring of large suspicious choroidal lesions. Seminars in ophthalmology 2009, 24, 43-44, doi:10.1080/08820530802520384.

50. Mackenzie, P.J.; Russell, M.; Ma, P.E.; Isbister, C.M.; Maberley, D.A. Sensitivity and specificity of the optos optomap for detecting peripheral retinal lesions. Retina (Philadelphia, Pa.) 2007, 27, 1119-1124, doi:10.1097/IAE.0b013e3180592b5c.

51. Lamirel, C.; Bruce, B.B.; Wright, D.W.; Newman, N.J.; Biousse, V. Nonmydriatic digital ocular fundus photography on the iPhone 3G: the FOTO-ED study. Archives of ophthalmology (Chicago, III. : 1960) 2012, 130, 939-940, doi:10.1001/archophthalmol.2011.2488.

52. Kumar, S.; Wang, E.H.; Pokabla, M.J.; Noecker, R.J. Teleophthalmology assessment of diabetic retinopathy fundus images: smartphone versus standard office computer workstation. Telemedicine journal and e-health : the official journal of the American Telemedicine Association 2012, 18, 158-162, doi:10.1089/tmj.2011.0089.

53. Bastawrous, A.; Giardini, M.E.; Bolster, N.M.; Peto, T.; Shah, N.; Livingstone, I.A.; Weiss, H.A.; Hu, S.; Rono, H.; Kuper, H., et al. Clinical Validation of a Smartphone-Based Adapter for Optic Disc Imaging in Kenya. JAMA ophthalmology 2016, 134, 151-158, doi:10.1001/jamaophthalmol.2015.4625.

54. Kivelä, T. Diagnosis of uveal melanoma. Dev Ophthalmol 2012, 49, 1-15, doi:10.1159/000330613.

55. Bakri, S.J.; Sculley, L.; Singh, A.D. Imaging techniques for uveal melanoma. Int Ophthalmol Clin 2006, 46, 113, doi:10.1097/01.iio.0000195859.86805.64.

56. Tarlan, B.; Kıratlı, H. Uveal Melanoma: Current Trends in Diagnosis and Management. Turkish journal of ophthalmology 2016, 46, 123-137, doi:10.4274/tjo.37431.

57. Hurley, B.R.; Regillo, C.D. Fluorescein Angiography: General Principles and Interpretation. In Retinal Angiography and Optical Coherence Tomography, Arevalo, J.F., Ed. Springer New York: New York, NY, 2009; 10.1007/978-0-387-68987-6_2pp. 27-42.

58. Dithmar S (Stefan), H.F. Fluorescence angiography in ophthalmology.; Heidelberg: Springer Medizin Verlag; : 2008.

59. Meyer, K.; Augsburger, J.J. Independent diagnostic value of fluorescein angiography in the evaluation of intraocular tumors. Graefe's archive for clinical and experimental ophthalmology = Albrecht von Graefes Archiv fur klinische und experimentelle Ophthalmologie 1999, 237, 489-494, doi:10.1007/s004170050267.

60. Kawali, A.; Pichi, F.; Avadhani, K.; Invernizzi, A.; Hashimoto, Y.; Mahendradas, P. Multimodal Imaging of the Normal Eye. Ocular immunology and inflammation 2017, 25, 721-731, doi:10.1080/09273948.2017.1375531.

61. Kapoor, A.; Beniwal, V.; Beniwal, S.; Mathur, H.; Kumar, H.S. Management of uveal tract melanoma: A comprehensive review. Journal of the Egyptian National Cancer Institute 2016, 28, 65-72, doi:10.1016/j.jnci.2016.02.003. 
62. Invernizzi, A.; Pellegrini, M.; Cornish, E.; Yi Chong Teo, K.; Cereda, M.; Chabblani, J. Imaging the Choroid: From Indocyanine Green Angiography to Optical Coherence Tomography Angiography. Asia-Pacific journal of ophthalmology (Philadelphia, Pa.) 2020, 9, 335-348, doi:10.1097/apo.0000000000000307.

63. Shields, C.L.; Shields, J.A.; De Potter, P. Patterns of indocyanine green videoangiography of choroidal tumours. The British journal of ophthalmology 1995, 79, 237-245, doi:10.1136/bjo.79.3.237.

64. HyvÄRinen, L.E.A.; Flower, R.W. INDOCYANINE GREEN FLUORESCENCE ANGIOGRAPHY. Acta Ophthalmologica 1980, 58, 528-538, doi:https://doi.org/10.1111/j.1755-3768.1980.tb08294.x.

65. Sato, T.; Karasawa, Y.; Ishikawa, S.; Taguchi, M.; Muraoka, T.; Ito, M.; Takeuchi, M. Potential Phototoxicity of Indocyanine Green in Retinal Pigment Epithelial Cells after Angiography under Ambient Illumination. Oxidative medicine and cellular longevity 2018, 2018, 6065285, doi:10.1155/2018/6065285.

66. Lavinsky, D.; Belfort, R.N.; Navajas, E.; Torres, V.; Martins, M.C.; Belfort, R., Jr. Fundus autofluorescence of choroidal nevus and melanoma. The British journal of ophthalmology 2007, 91, 1299-1302, doi:10.1136/bjo.2007.116665.

67. Almeida, A.; Kaliki, S.; Shields, C.L. Autofluorescence of intraocular tumours. Current opinion in ophthalmology 2013, 24, 222-232, doi:10.1097/ICU.0b013e32835f8ba1.

68. Ly, A.; Nivison-Smith, L.; Assaad, N.; Kalloniatis, M. Fundus Autofluorescence in Age-related Macular Degeneration. Optom Vis Sci 2017, 94, 246-259, doi:10.1097/OPX.0000000000000997.

69. Silverman, R.H. Focused ultrasound in ophthalmology. Clin Ophthalmol 2016, 10, 1865-1875, doi:10.2147/opth.S99535.

70. Gonzalez, E.M.; Rodriguez, A.; Garcia, I. Review of ocular ultrasonography. Vet Radiol Ultrasound 2001, 42, 485-495, doi:10.1111/j.1740-8261.2001.tb00975.x.

71. Oberg, J.; Spenger, C.; Wang, F.H.; Andersson, A.; Westman, E.; Skoglund, P.; Sunnemark, D.; Norinder, U.; Klason, T.; Wahlund, L.O., et al. Age related changes in brain metabolites observed by $1 \mathrm{H}$ MRS in APP/PS1 mice. Neurobiol Aging 2008, 29, 1423-1433, doi:10.1016/j.neurobiolaging.2007.03.002.

72. Lin, D.T.; Munk, P.L.; Maberley, A.L.; Cooperberg, P.L.; Rootman, J. Ultrasonography of pathologically proved choroidal melanoma with a high-resolution small-parts scanner. Can J Ophthalmol 1987, 22, $161-164$.

73. Coleman, D.J. Ocular tumor patterns. Bibl Ophthalmol 1975, 136-140.

74. Blanco, G. Diagnosis and treatment of orbital invasion in uveal melanoma. Can J Ophthalmol 2004, 39, 388396, doi:10.1016/s0008-4182(04)80010-3.

75. Hosten, N.; Bornfeld, N.; Wassmuth, R.; Lemke, A.J.; Sander, B.; Bechrakis, N.E.; Felix, R. Uveal melanoma: detection of extraocular growth with MR imaging and US. Radiology 1997, 202, 61-67, doi:10.1148/radiology.202.1.8988193.

76. Xu, G.; Xue, Y.; Özkurt, Z.G.; Slimani, N.; Hu, Z.; Wang, X.; Xia, K.; Ma, T.; Zhou, Q.; Demirci, H. Photoacoustic imaging features of intraocular tumors: Retinoblastoma and uveal melanoma. PLoS One 2017, 12, e0170752, doi:10.1371/journal.pone.0170752.

77. Anon. Availabe online: https://www.mdsave.com/procedures/ultrasound/d781f5ca (accessed on 08-022021).

78. Silverman, R.H. High-resolution ultrasound imaging of the eye - a review. Clin Exp Ophthalmol 2009, 37, 5467, doi:10.1111/j.1442-9071.2008.01892.x.

79. Nanji, A.A.; Mercado, C.; Galor, A.; Dubovy, S.; Karp, C.L. Updates in Ocular Surface Tumor Diagnostics. Int Ophthalmol Clin 2017, 57, 47-62, doi:10.1097/iio.0000000000000174.

80. Scott, I.U.; Murray, T.G.; Hughes, J.R. Evaluation of imaging techniques for detection of extraocular extension of choroidal melanoma. Arch Ophthalmol 1998, 116, 897-899, doi:10.1001/archopht.116.7.897. 
81. Martin, J.A.; Robertson, D.M. Extrascleral extension of choroidal melanoma diagnosed by ultrasound. Ophthalmology 1983, 90, 1554-1559, doi:10.1016/s0161-6420(83)34349-9.

82. Bedi, D.G.; Gombos, D.S.; Ng, C.S.; Singh, S. Sonography of the eye. AJR Am J Roentgenol 2006, 187, 10611072, doi:10.2214/ajr.04.1842.

83. Damianidis, C.H.; Konstantinou, D.; Kyriakou, V.; Arvaniti, M.; Kotziamani, N.; Rodokalakis, G.; Agriou, A.; Emmanouilidou, M.; Tsitouridis, I. Magnetic resonance imaging and ultrasonographic evaluation of retinal detachment in orbital uveal melanomas. Neuroradiol J 2010, 23, 329-338, doi:10.1177/197140091002300313.

84. Grech Fonk, L.; Ferreira, T.A.; Webb, A.G.; Luyten, G.P.M.; Beenakker, J.M. The Economic Value of MRImaging for Uveal Melanoma. Clin Ophthalmol 2020, 14, 1135-1143, doi:10.2147/opth.S238405.

85. Sobottka, B.; Kreissig, I. Ultrasonography of metastases and melanomas of the choroid. Curr Opin Ophthalmol 1999, 10, 164-167, doi:10.1097/00055735-199906000-00002.

86. Walter, U.; Niendorf, T.; Graessl, A.; Rieger, J.; Krüger, P.C.; Langner, S.; Guthoff, R.F.; Stachs, O. Ultrahigh field magnetic resonance and colour Doppler real-time fusion imaging of the orbit--a hybrid tool for assessment of choroidal melanoma. Eur Radiol 2014, 24, 1112-1117, doi:10.1007/s00330-014-3101-5.

87. Konstantopoulos, A.; Hossain, P.; Anderson, D.F. Recent advances in ophthalmic anterior segment imaging: a new era for ophthalmic diagnosis? Br J Ophthalmol 2007, 91, 551-557, doi:10.1136/bjo.2006.103408.

88. Aumann, S.; Donner, S.; Fischer, J.; Müller, F. Optical Coherence Tomography (OCT): Principle and Technical Realization. In High Resolution Imaging in Microscopy and Ophthalmology: New Frontiers in Biomedical Optics, Bille, J.F., Ed. Springer

Copyright 2019, The Author(s). Cham (CH), 2019; 10.1007/978-3-030-16638-0_3pp. 59-85.

89. JERNIGAN, R.C. Availabe online: https://www.photonics.com/a36339/Is OCT Worth It (accessed on 11.02.2021).

90. Popescu, D.P.; Choo-Smith, L.P.; Flueraru, C.; Mao, Y.; Chang, S.; Disano, J.; Sherif, S.; Sowa, M.G. Optical coherence tomography: fundamental principles, instrumental designs and biomedical applications.

Biophysical reviews 2011, 3, 155, doi:10.1007/s12551-011-0054-7.

91. Nanji, A.A.; Sayyad, F.E.; Galor, A.; Dubovy, S.; Karp, C.L. High-Resolution Optical Coherence Tomography as an Adjunctive Tool in the Diagnosis of Corneal and Conjunctival Pathology. Ocul Surf 2015, 13, 226-235, doi:10.1016/j.jtos.2015.02.001.

92. Thomas, B.J.; Galor, A.; Nanji, A.A.; El Sayyad, F.; Wang, J.; Dubovy, S.R.; Joag, M.G.; Karp, C.L. Ultra highresolution anterior segment optical coherence tomography in the diagnosis and management of ocular surface squamous neoplasia. The ocular surface 2014, 12, 46-58, doi:10.1016/j.jtos.2013.11.001.

93. Muscat, S.; Srinivasan, S.; Sampat, V.; Kemp, E.; Parks, S.; Keating, D. Optical coherence tomography in the diagnosis of subclinical serous detachment of the macula secondary to a choroidal nevus. Ophthalmic Surg Lasers 2001, 32, 474-476.

94. Torres, V.L.; Brugnoni, N.; Kaiser, P.K.; Singh, A.D. Optical coherence tomography enhanced depth imaging of choroidal tumors. Am J Ophthalmol 2011, 151, 586-593.e582, doi:10.1016/j.ajo.2010.09.028.

95. Say, E.A.T.; Ferenczy, S.; Magrath, G.N.; Samara, W.A.; Khoo, C.T.L.; Shields, C.L. IMAGE QUALITY AND ARTIFACTS ON OPTICAL COHERENCE TOMOGRAPHY ANGIOGRAPHY: Comparison of Pathologic and Paired Fellow Eyes in 65 Patients With Unilateral Choroidal Melanoma Treated With Plaque Radiotherapy. Retina (Philadelphia, Pa.) 2017, 37, 1660-1673, doi:10.1097/iae.0000000000001414. 
96. Brinkmann, M.P.; Kibele, N.X.; Prasuhn, M.; Kakkassery, V.; Toro, M.D.; Ranjbar, M.; Grisanti, S.; Becker, M.; Rommel, F. Evaluating Retinal and Choroidal Perfusion Changes after Isometric and Dynamic Activity Using Optical Coherence Tomography Angiography. Diagnostics (Basel, Switzerland) 2021, 11, doi:10.3390/diagnostics11050808.

97. Bonfiglio, V.; Ortisi, E.; Scollo, D.; Reibaldi, M.; Russo, A.; Pizzo, A.; Faro, G.; Macchi, I.; Fallico, M.; Toro, M.D., et al. Vascular changes after vitrectomy for rhegmatogenous retinal detachment: optical coherence tomography angiography study. Acta ophthalmologica 2019, 10.1111/aos.14315, doi:10.1111/aos.14315.

98. Wrzesińska, D.; Nowomiejska, K.; Nowakowska, D.; Toro, M.D.; Bonfiglio, V.; Reibaldi, M.; Avitabile, T.; Rejdak, R. Secondary Vitrectomy with Internal Limiting Membrane Plug due to Persistent Full-Thickness Macular Hole OCT-Angiography and Microperimetry Features: Case Series. Journal of ophthalmology 2020, 2020, 2650873, doi:10.1155/2020/2650873.

99. Neroev, V.V.; Saakyan, S.V.; Myakoshina, E.B.; Okhotsimskaya, T.D.; Fadeeva, V.A. [Role of optical coherence tomography angiography in diagnostics of early choroidal melanoma and circumscribed choroidal hemangioma]. Vestnik oftalmologii 2018, 134, 4-18, doi:10.17116/oftalma201813434.

100. Ghassemi, F.; Mirshahi, R.; Fadakar, K.; Sabour, S. Optical coherence tomography angiography in choroidal melanoma and nevus. Clin Ophthalmol 2018, 12, 207-214, doi:10.2147/opth.s148897.

101. Valverde-Megías, A.; Say, E.A.; Ferenczy, S.R.; Shields, C.L. DIFFERENTIAL MACULAR FEATURES ON OPTICAL COHERENCE TOMOGRAPHY ANGIOGRAPHY IN EYES WITH CHOROIDAL NEVUS AND MELANOMA. Retina (Philadelphia, Pa.) 2017, 37, 731-740, doi:10.1097/iae.0000000000001233.

102. de Carlo, T.E.; Mieler, W.F. Automated quantitative OCTA measurements of uveal melanoma-treated eyes with and without radiation retinopathy after I-125 plaque therapy and of nonirradiated fellow eyes. Eye (Lond) 2021, 35, 769-776, doi:10.1038/s41433-020-01237-8.

103. Skalet, A.H.; Liu, L.; Binder, C.; Miller, A.K.; Crilly, R.; Hung, A.Y.; Wilson, D.J.; Huang, D.; Jia, Y. Longitudinal Detection of Radiation-Induced Peripapillary and Macular Retinal Capillary Ischemia Using OCT Angiography. Ophthalmology. Retina 2020, 4, 320-326, doi:10.1016/j.oret.2019.10.001.

104. Skalet, A.H.; Liu, L.; Binder, C.; Miller, A.K.; Wang, J.; Wilson, D.J.; Crilly, R.; Thomas, C.R., Jr.; Hung, A.Y.; Huang, D., et al. Quantitative OCT Angiography Evaluation of Peripapillary Retinal Circulation after Plaque Brachytherapy. Ophthalmology. Retina 2018, 2, 244-250, doi:10.1016/j.oret.2017.06.005.

105. Matet, A.; Daruich, A.; Zografos, L. Radiation Maculopathy After Proton Beam Therapy for Uveal Melanoma: Optical Coherence Tomography Angiography Alterations Influencing Visual Acuity. Invest Ophthalmol Vis Sci 2017, 58, 3851-3861, doi:10.1167/iovs.17-22324.

106. Yang, J.Y.; Wang, Q.; Chen, M.X.; Yan, Y.N.; Zhou, W.J.; Liu, Y.M.; Wei, W.B. Retinal Microvascular Changes in Uveal Melanoma Following Conbercept Injection after Plaque Radiotherapy as Detected By Optical Coherence Tomographic Angiography. Retina (Philadelphia, Pa.) 2021, 10.1097/iae.0000000000003236, doi:10.1097/iae.0000000000003236.

107. Bianciotto, C.; Shields, C.L.; Guzman, J.M.; Romanelli-Gobbi, M.; Mazzuca, D., Jr.; Green, W.R.; Shields, J.A. Assessment of anterior segment tumors with ultrasound biomicroscopy versus anterior segment optical coherence tomography in 200 cases. Ophthalmology 2011, 118, 1297-1302, doi:10.1016/j.ophtha.2010.11.011.

108. Pavlin, C.J.; Vásquez, L.M.; Lee, R.; Simpson, E.R.; Ahmed, II. Anterior segment optical coherence tomography and ultrasound biomicroscopy in the imaging of anterior segment tumors. American journal of ophthalmology 2009, 147, 214-219.e212, doi:10.1016/j.ajo.2008.08.023. 
109. Mlynárik, V. Introduction to nuclear magnetic resonance. Anal Biochem 2017, 529, 4-9, doi:10.1016/j.ab.2016.05.006.

110. Chambers, R.B.; Davidorf, F.H.; McAdoo, J.F.; Chakeres, D.W. Magnetic resonance imaging of uveal melanomas. Arch Ophthalmol 1987, 105, 917-921, doi:10.1001/archopht.1987.01060070053027.

111. Crooks, L.E.; Mills, C.M.; Davis, P.L.; Brant-Zawadzki, M.; Hoenninger, J.; Arakawa, M.; Watts, J.; Kaufman, L. Visualization of cerebral and vascular abnormalities by NMR imaging. The effects of imaging parameters on contrast. Radiology 1982, 144, 843-852, doi:10.1148/radiology.144.4.7111736.

112. Fiedorowicz, M.; Wełniak-Kamińska, M.; Świątkiewicz, M.; Orzeł, J.; Chorągiewicz, T.; Toro, M.D.; Rejdak, R.; Bogorodzki, P.; Grieb, P. Changes of Ocular Dimensions as a Marker of Disease Progression in a Murine Model of Pigmentary Glaucoma. Frontiers in Pharmacology 2020, 11, doi:10.3389/fphar.2020.573238.

113. Richdale, K.; Wassenaar, P.; Teal Bluestein, K.; Abduljalil, A.; Christoforidis, J.A.; Lanz, T.; Knopp, M.V.; Schmalbrock, P. 7 Tesla MR imaging of the human eye in vivo. J Magn Reson Imaging 2009, 30, 924-932, doi:10.1002/jmri.21959.

114. Beenakker, J.W.; Ferreira, T.A.; Soemarwoto, K.P.; Genders, S.W.; Teeuwisse, W.M.; Webb, A.G.; Luyten, G.P. Clinical evaluation of ultra-high-field MRI for three-dimensional visualisation of tumour size in uveal melanoma patients, with direct relevance to treatment planning. Magma (New York, N.Y.) 2016, 29, 571577, doi:10.1007/s10334-016-0529-4.

115. Hosten, N.; Lemke, A.J.; Bornfeld, N.; Wassmuth, R.; Schweiger, U.; Terstegge, K.; Felix, R. Fast spin-echo MR imaging of the eye. Eur Radiol 1996, 6, 900-903, doi:10.1007/bf00240700.

116. Houle, V.; Bélair, M.; Allaire, G.S. AIRP best cases in radiologic-pathologic correlation: choroidal melanoma. Radiographics 2011, 31, 1231-1236, doi:10.1148/rg.315105211.

117. Hirunpat, P.; Sanghan, N.; Hirunpat, S. White matter: A good reference for the signal intensity evaluation in magnetic resonance imaging for the diagnosis of uveal melanoma. Neuroradiol J 2020, 10.1177/1971400920973407, 1971400920973407, doi:10.1177/1971400920973407.

118. Sullivan, J.A.; Harms, S.E. Surface-coil MR imaging of orbital neoplasms. AJNR Am J Neuroradiol 1986, 7, 2934.

119. de Keizer, R.J.; Vielvoye, G.J.; de Wolff-Rouendaal, D. Nuclear magnetic resonance imaging of intraocular tumors. Am J Ophthalmol 1986, 102, 438-441, doi:10.1016/0002-9394(86)90070-x.

120. Commoner, B.; Townsend, J.; Pake, G.E. Free radicals in biological materials. Nature 1954, 174, 689-691, doi:10.1038/174689a0.

121. Gomori, J.M.; Grossman, R.I.; Shields, J.A.; Augsburger, J.J.; Joseph, P.M.; DeSimeone, D. Choroidal melanomas: correlation of NMR spectroscopy and MR imaging. Radiology 1986, 158, 443-445, doi:10.1148/radiology.158.2.3941871.

122. Tailor, T.D.; Gupta, D.; Dalley, R.W.; Keene, C.D.; Anzai, Y. Orbital neoplasms in adults: clinical, radiologic, and pathologic review. Radiographics 2013, 33, 1739-1758, doi:10.1148/rg.336135502.

123. Mafee, M.F.; Peyman, G.A.; Grisolano, J.E.; Fletcher, M.E.; Spigos, D.G.; Wehrli, F.W.; Rasouli, F.; Capek, V. Malignant uveal melanoma and simulating lesions: MR imaging evaluation. Radiology 1986, 160, 773-780, doi:10.1148/radiology.160.3.3737917.

124. Stroszczynski, C.; Hosten, N.; Bornfeld, N.; Wiegel, T.; Schueler, A.; Foerster, P.; Lemke, A.J.; Hoffmann, K.T.; Felix, R. Choroidal hemangioma: MR findings and differentiation from uveal melanoma. AJNR Am J Neuroradiol 1998, 19, 1441-1447. 
125. Schueler, A.O.; Hosten, N.; Bechrakis, N.E.; Lemke, A.J.; Foerster, P.; Felix, R.; Foerster, M.H.; Bornfeld, N. High resolution magnetic resonance imaging of retinoblastoma. Br J Ophthalmol 2003, 87, 330-335, doi:10.1136/bjo.87.3.330.

126. Jacobsen, B.H.; Ricks, C.; Harrie, R.P. Ocular ultrasound versus MRI in the detection of extrascleral extension in a patient with choroidal melanoma. BMC Ophthalmol 2018, 18, 320, doi:10.1186/s12886-018-0990-0.

127. Récsán, Z.; Karlinger, K.; Fodor, M.; Zalatnai, A.; Papp, M.; Salacz, G. MRI for the evaluation of scleral invasion and extrascleral extension of uveal melanomas. Clin Radiol 2002, 57, 371-376, doi:10.1053/crad.2001.0859.

128. Beenakker, J.W.; van Rijn, G.A.; Luyten, G.P.; Webb, A.G. High-resolution MRI of uveal melanoma using a microcoil phased array at 7 T. NMR Biomed 2013, 26, 1864-1869, doi:10.1002/nbm.3041.

129. Lindner, T.; Langner, S.; Falke, K.; Walter, U.; Krüger, P.C.; Pohlmann, A.; Zimpfer, A.; Stahnke, T.; Hadlich, S.; Guthoff, R., et al. Anatomic and pathological characterization of choroidal melanoma using multimodal imaging: what is practical, what is needed? Melanoma research 2015, 25, 252-258, doi:10.1097/cmr.0000000000000156.

130. Simon, E.M.; McCaffery, S.; Rowley, H.A.; Fischbein, N.J.; Shimikawa, A.; O'Brien, J.M. High-resolution 3D T2weighted fast spin echo: new applications in the orbit. Neuroradiology 2003, 45, 489-492, doi:10.1007/s00234-003-0954-8.

131. Fanea, L.; Fagan, A.J. Review: magnetic resonance imaging techniques in ophthalmology. Mol Vis 2012, 18, 2538-2560.

132. Bert, R.J.; Patz, S.; Ossiani, M.; Caruthers, S.D.; Jara, H.; Krejza, J.; Freddo, T. High-resolution MR imaging of the human eye 2005. Acad Radiol 2006, 13, 368-378, doi:10.1016/j.acra.2005.10.023.

133. Marshall, E.; Romaniuk, C.; Ghaneh, P.; Wong, H.; McKay, M.; Chopra, M.; Coupland, S.E.; Damato, B.E. MRI in the detection of hepatic metastases from high-risk uveal melanoma: a prospective study in 188 patients. The British journal of ophthalmology 2013, 97, 159-163, doi:10.1136/bjophthalmol-2012-302323.

134. Servois, V.; Mariani, P.; Malhaire, C.; Petras, S.; Piperno-Neumann, S.; Plancher, C.; Levy-Gabriel, C.; Lumbroso-le Rouic, L.; Desjardins, L.; Salmon, R.J. Preoperative staging of liver metastases from uveal melanoma by magnetic resonance imaging (MRI) and fluorodeoxyglucose-positron emission tomography (FDG-PET). European journal of surgical oncology : the journal of the European Society of Surgical Oncology and the British Association of Surgical Oncology 2010, 36, 189-194, doi:10.1016/j.ejso.2009.08.010.

135. Francis, J.H.; Catalanotti, F.; Landa, J.; Barker, C.A.; Shoushtari, A.N.; Abramson, D.H. Hepatic abnormalities identified by staging MRI and accuracy of MRI of patients with uveal melanoma. The British journal of ophthalmology 2019, 103, 1266-1271, doi:10.1136/bjophthalmol-2018-312612.

136. Ferreira, T.A.; Grech Fonk, L.; Jaarsma-Coes, M.G.; van Haren, G.G.R.; Marinkovic, M.; Beenakker, J.M. MRI of Uveal Melanoma. Cancers 2019, 11, doi:10.3390/cancers11030377.

137. Jaarsma-Coes, M.G.; Goncalves Ferreira, T.A.; van Haren, G.R.; Marinkovic, M.; Beenakker, J.M. MRI enables accurate diagnosis and follow-up in uveal melanoma patients after vitrectomy. Melanoma research 2019, 29, 655-659, doi:10.1097/cmr.0000000000000568.

138. Wong, T.; Lo, L.W.; Fung, P.Y.; Lai, H.Y.; She, H.L.; Ng, W.K.; Kwok, K.M.; Lee, C.M. Magnetic resonance imaging of breast augmentation: a pictorial review. Insights into imaging 2016, 7, 399-410, doi:10.1007/s13244-016-0482-9.

139. Chang, A.E.; Karnell, L.H.; Menck, H.R. The National Cancer Data Base report on cutaneous and noncutaneous melanoma: a summary of 84,836 cases from the past decade. The American College of Surgeons Commission on Cancer and the American Cancer Society. Cancer 1998, 83, 1664-1678, doi:10.1002/(sici)1097-0142(19981015)83:8<1664::aid-cncr23>3.0.co;2-g. 
140. Erb-Eigner, K.; Willerding, G.; Taupitz, M.; Hamm, B.; Asbach, P. Diffusion-weighted imaging of ocular melanoma. Investigative radiology 2013, 48, 702-707, doi:10.1097/RLI.0b013e31828eea67.

141. Ferreira, T.A.; Grech Fonk, L.; Jaarsma-Coes, M.G.; van Haren, G.G.R.; Marinkovic, M.; Beenakker, J.-W.M. MRI of Uveal Melanoma. Cancers 2019, 11, doi:10.3390/cancers11030377.

142. Kamrava, M.; Sepahdari, A.R.; Leu, K.; Wang, P.C.; Roberts, K.; Demanes, D.J.; McCannel, T.; Ellingson, B.M. Quantitative multiparametric MRI in uveal melanoma: increased tumor permeability may predict monosomy 3. Neuroradiology 2015, 57, 833-840, doi:10.1007/s00234-015-1546-0.

143. Beenakker, J.-W.M.; Ferreira, T.A.; Soemarwoto, K.P.; Genders, S.W.; Teeuwisse, W.M.; Webb, A.G.; Luyten, G.P.M. Clinical evaluation of ultra-high-field MRI for three-dimensional visualisation of tumour size in uveal melanoma patients, with direct relevance to treatment planning. Magnetic Resonance Materials in Physics, Biology and Medicine 2016, 29, 571-577, doi:10.1007/s10334-016-0529-4.

144. Ciller, C.; De Zanet, S.I.; Rüegsegger, M.B.; Pica, A.; Sznitman, R.; Thiran, J.P.; Maeder, P.; Munier, F.L.; Kowal, J.H.; Cuadra, M.B. Automatic Segmentation of the Eye in 3D Magnetic Resonance Imaging: A Novel Statistical Shape Model for Treatment Planning of Retinoblastoma. International journal of radiation oncology, biology, physics 2015, 92, 794-802, doi:10.1016/j.ijrobp.2015.02.056.

145. Foti, P.V.; Longo, A.; Reibaldi, M.; Russo, A.; Privitera, G.; Spatola, C.; Raffaele, L.; Salamone, V.; Farina, R.; Palmucci, S., et al. Uveal melanoma: quantitative evaluation of diffusion-weighted MR imaging in the response assessment after proton-beam therapy, long-term follow-up. La radiologia medica 2017, 122, 131139, doi:10.1007/s11547-016-0697-3.

146. Mazonakis, M.; Damilakis, J. Computed tomography: What and how does it measure? European journal of radiology 2016, 85, 1499-1504, doi:10.1016/j.ejrad.2016.03.002.

147. Eguren, M.; Holguin, A.; Diaz, K.; Vidalon, J.; Linan, C.; Pereira, C.; Lagravere Vich, M.O. Can gray values be converted to Hounsfield units? A systematic review. Dento maxillo facial radiology 2021, 10.1259/dmfr.20210140, 20210140, doi:10.1259/dmfr.20210140.

148. Blackham, K.A.; Vidal, L.L.M. Computed Tomography. In Encyclopedia of the Neurological Sciences (Second Edition), Aminoff, M.J., Daroff, R.B., Eds. Academic Press: Oxford, 2014; https://doi.org/10.1016/B978-0-12385157-4.00194-9pp. 848-853.

149. Naik, M.N.; Tourani, K.L.; Sekhar, G.C.; Honavar, S.G. Interpretation of computed tomography imaging of the eye and orbit. A systematic approach. Indian journal of ophthalmology 2002, 50, 339-353.

150. de Keizer, R.J.; Vielvoye, G.J.; de Wolff-Rouendaal, D. CT-scan and intraocular tumours: detection and assessment of size and extrascleral growth of uveal melanomas. Documenta ophthalmologica. Advances in ophthalmology 1986, 61, 285-294, doi:10.1007/bf00142355.

151. Lin, E.; Alessio, A. What are the basic concepts of temporal, contrast, and spatial resolution in cardiac CT? Journal of cardiovascular computed tomography 2009, 3, 403-408, doi:10.1016/j.jcct.2009.07.003.

152. Mafee, M.F.; Peyman, G.A.; McKusick, M.A. Malignant uveal melanoma and similar lesions studied by computed tomography. Radiology 1985, 156, 403-408, doi:10.1148/radiology.156.2.4011902.

153. Sallet, G.; Serop, S.; Verbraeken, H.; Hanssens, M. The value of medical imaging techniques in the diagnosis of extra-ocular extension of malign melanoma of the choroid: a case report. Bulletin de la Societe belge d'ophtalmologie 1993, 248, 53-58.

154. Khan, S.N.; Sepahdari, A.R. Orbital masses: CT and MRI of common vascular lesions, benign tumors, and malignancies. Saudi J Ophthalmol 2012, 26, 373-383, doi:10.1016/j.sjopt.2012.08.001. 
155. Kurli, M.; Reddy, S.; Tena, L.B.; Pavlick, A.C.; Finger, P.T. Whole body positron emission tomography/computed tomography staging of metastatic choroidal melanoma. American journal of ophthalmology 2005, 140, 193-199, doi:10.1016/j.ajo.2005.02.051.

156. Holly, T.A.; Abbott, B.G.; Al-Mallah, M.; Calnon, D.A.; Cohen, M.C.; DiFilippo, F.P.; Ficaro, E.P.; Freeman, M.R.; Hendel, R.C.; Jain, D., et al. Single photon-emission computed tomography. Journal of Nuclear Cardiology 2010, 17, 941-973, doi:10.1007/s12350-010-9246-y.

157. Wernick, M.N.; Aarsvold, J.N. CHAPTER 2 - Introduction to Emission Tomography. In Emission Tomography, Wernick, M.N., Aarsvold, J.N., Eds. Academic Press: San Diego, 2004; https://doi.org/10.1016/B978012744482-6.50005-3pp. 11-23.

158. Nolte, T.; Gross-Weege, N.; Schulz, V. (Hybrid) SPECT and PET Technologies. Recent results in cancer research. Fortschritte der Krebsforschung. Progres dans les recherches sur le cancer 2020, 216, 111-133, doi:10.1007/978-3-030-42618-7_3.

159. Alonso, O.; Núñez, M.; Cánepa, J.; Guisoli, P.; Mut, F.; Lago, G.; Touya, E. Evaluation of ocular tumors with technetium-99m-MIBI: planar pinhole technique or SPECT? Journal of nuclear medicine technology 2000, 28, 85-87.

160. Abe, K.; Sasaki, M.; Koga, H.; Kaneko, K.; Sawamoto, H.; Yoshikawa, H.; Honda, H. Clinical role of 123I-IMP SPECT for the differential diagnosis of ocular malignant melanoma: a time-course analysis. Nuclear medicine communications 2007, 28, 567-573, doi:10.1097/MNM.0b013e328194f221.

161. Goto, H. Clinical efficacy of 123I-IMP SPECT for the diagnosis of malignant uveal melanoma. International journal of clinical oncology 2004, 9, 74-78, doi:10.1007/s10147-003-0380-2.

162. Kato, K.; Kubota, T.; Ikeda, M.; Tadokoro, M.; Abe, S.; Nakano, S.; Nishino, M.; Kobayashi, H.; Ishigaki, T. Low efficacy of 18F-FDG PET for detection of uveal malignant melanoma compared with 123I-IMP SPECT. Journal of nuclear medicine : official publication, Society of Nuclear Medicine 2006, 47, 404-409.

163. Yamazaki, K.; Nishii, R.; Maeda, T.; Makishima, H.; Kasuya, G.; Chang, T.; Tamura, K.; Wakatsuki, M.; Tsuji, H.; Murakami, K., et al. Assessment of SPECT-CT fusion images and semi-quantitative evaluation using SUV in (123)I-IMP SPECT in patients with choroidal melanoma. Annals of nuclear medicine 2020, 34, 864-872, doi:10.1007/s12149-020-01517-z.

164. Anon. Availabe online: https://www.mdsave.com/procedures/brain-spect/d785ffc9 (accessed on 10.02.2021).

165. Brooks, D.J. Positron emission tomography and single-photon emission computed tomography in central nervous system drug development. NeuroRx : the journal of the American Society for Experimental NeuroTherapeutics 2005, 2, 226-236, doi:10.1602/neurorx.2.2.226.

166. Salvatori, M.; Rizzo, A.; Rovera, G.; Indovina, L.; Schillaci, O. Radiation dose in nuclear medicine: the hybrid imaging. La Radiologia medica 2019, 124, 768-776, doi:10.1007/s11547-019-00989-y.

167. Kalemaki, M.S.; Karantanas, A.H.; Exarchos, D.; Detorakis, E.T.; Zoras, O.; Marias, K.; Millo, C.; Bagci, U.; Pallikaris, I.; Stratis, A., et al. PET/CT and PET/MRI in ophthalmic oncology (Review). International journal of oncology 2020, 56, 417-429, doi:10.3892/ijo.2020.4955.

168. Omami, G.; Tamimi, D.; Branstetter, B.F. Basic principles and applications of (18)F-FDG-PET/CT in oral and maxillofacial imaging: A pictorial essay. Imaging science in dentistry 2014, 44, 325-332, doi:10.5624/isd.2014.44.4.325.

169. Moses, W.W. Fundamental Limits of Spatial Resolution in PET. Nuclear instruments \& methods in physics research. Section A, Accelerators, spectrometers, detectors and associated equipment 2011, 648 Supplement 1, s236-s240, doi:10.1016/j.nima.2010.11.092. 
170. Hui, K.H.; Pfeiffer, M.L.; Esmaeli, B. Value of positron emission tomography/computed tomography in diagnosis and staging of primary ocular and orbital tumors. Saudi journal of ophthalmology : official journal of the Saudi Ophthalmological Society 2012, 26, 365-371, doi:10.1016/j.sjopt.2012.08.008.

171. Griffeth, L.K. Use of PET/CT scanning in cancer patients: technical and practical considerations. Proceedings (Baylor University. Medical Center) 2005, 18, 321-330, doi:10.1080/08998280.2005.11928089.

172. Kapoor, V.; McCook, B.M.; Torok, F.S. An introduction to PET-CT imaging. Radiographics : a review publication of the Radiological Society of North America, Inc 2004, 24, 523-543, doi:10.1148/rg.242025724.

173. Reddy, S.; Kurli, M.; Tena, L.B.; Finger, P.T. PET/CT imaging: detection of choroidal melanoma. The British journal of ophthalmology 2005, 89, 1265-1269, doi:10.1136/bjo.2005.066399.

174. Finger, P.T.; Chin, K.; lacob, C.E. 18-Fluorine-labelled 2-deoxy-2-fluoro-D-glucose positron emission tomography/computed tomography standardised uptake values: a non-invasive biomarker for the risk of metastasis from choroidal melanoma. The British journal of ophthalmology 2006, 90, 1263-1266, doi:10.1136/bjo.2006.097949.

175. Matsuo, T.; Ogino, Y.; Ichimura, K.; Tanaka, T.; Kaji, M. Clinicopathological correlation for the role of fluorodeoxyglucose positron emission tomography computed tomography in detection of choroidal malignant melanoma. International journal of clinical oncology 2014, 19, 230-239, doi:10.1007/s10147-0130538-5.

176. McCannel, T.A.; Reddy, S.; Burgess, B.L.; Auerbach, M. Association of positive dual-modality positron emission tomography/computed tomography imaging of primary choroidal melanoma with chromosome 3 loss and tumor size. Retina (Philadelphia, Pa.) 2010, 30, 146-151, doi:10.1097/IAE.0b013e3181b32f36.

177. Papastefanou, V.P.; Islam, S.; Szyszko, T.; Grantham, M.; Sagoo, M.S.; Cohen, V.M. Metabolic activity of primary uveal melanoma on PET/CT scan and its relationship with monosomy 3 and other prognostic factors. The British journal of ophthalmology 2014, 98, 1659-1665, doi:10.1136/bjophthalmol-2014-305304.

178. Klingenstein, A.; Haug, A.R.; Nentwich, M.M.; Tiling, R.; Schaller, U.C. Whole-body F-18-fluoro-2deoxyglucose positron emission tomography/computed tomography imaging in the follow-up of metastatic uveal melanoma. Melanoma research 2010, 20, 511-516, doi:10.1097/CMR.0b013e3283403d6c.

179. Strobel, K.; Bode, B.; Dummer, R.; Veit-Haibach, P.; Fischer, D.R.; Imhof, L.; Goldinger, S.; Steinert, H.C.; von Schulthess, G.K. Limited value of 18F-FDG PET/CT and S-100B tumour marker in the detection of liver metastases from uveal melanoma compared to liver metastases from cutaneous melanoma. European journal of nuclear medicine and molecular imaging 2009, 36, 1774-1782, doi:10.1007/s00259-009-1175-0.

180. Orcurto, V.; Denys, A.; Voelter, V.; Schalenbourg, A.; Schnyder, P.; Zografos, L.; Leyvraz, S.; Delaloye, A.B.; Prior, J.O. (18)F-fluorodeoxyglucose positron emission tomography/computed tomography and magnetic resonance imaging in patients with liver metastases from uveal melanoma: results from a pilot study. Melanoma research 2012, 22, 63-69, doi:10.1097/CMR.0b013e32834d3dcb.

181. Foti, P.V.; Longo, A.; Reibaldi, M.; Russo, A.; Privitera, G.; Spatola, C.; Raffaele, L.; Salamone, V.; Farina, R.; Palmucci, S., et al. Uveal melanoma: quantitative evaluation of diffusion-weighted MR imaging in the response assessment after proton-beam therapy, long-term follow-up. La Radiologia medica 2017, 122, 131139, doi:10.1007/s11547-016-0697-3.

182. Anon. Availabe online: https://www.mdsave.com/procedures/pet-scan/d782facc (accessed on 10.02.2021

).

183. Huang, B.; Law, M.W.; Khong, P.L. Whole-body PET/CT scanning: estimation of radiation dose and cancer risk. Radiology 2009, 251, 166-174, doi:10.1148/radiol.2511081300. 
184. Núñez Miller, R.; Pozo, M.A. Non-FDG PET in oncology. Clinical \& translational oncology : official publication of the Federation of Spanish Oncology Societies and of the National Cancer Institute of Mexico 2011, 13, 780786, doi:10.1007/s12094-011-0733-7.

185. Eisenmenger, L.B. Non-FDG Radiopharmaceuticals in Head and Neck PET Imaging: Current Techniques and Future Directions. Seminars in ultrasound, CT, and MR 2019, 40, 424-433, doi:10.1053/j.sult.2019.07.006.

186. Bastawrous, S.; Bhargava, P.; Behnia, F.; Djang, D.S.; Haseley, D.R. Newer PET application with an old tracer: role of 18F-NaF skeletal PET/CT in oncologic practice. Radiographics 2014, 34, 1295-1316, doi:10.1148/rg.345130061.

187. Liu, Y.; Sheng, J.; Dong, Z.; Xu, Y.; Huang, Q.; Pan, D.; Wang, L.; Yang, M. The diagnostic performance of (18)Ffluoride PET/CT in bone metastases detection: a meta-analysis. Clin Radiol 2019, 74, 196-206, doi:10.1016/j.crad.2018.12.011.

188. Shen, C.T.; Qiu, Z.L.; Han, T.T.; Luo, Q.Y. Performance of 18F-fluoride PET or PET/CT for the detection of bone metastases: a meta-analysis. Clinical nuclear medicine 2015, 40, 103-110, doi:10.1097/rlu.0000000000000592.

189. Feng, H.; Xia, X.; Li, C.; Song, Y.; Qin, C.; Liu, Q.; Zhang, Y.; Lan, X. Imaging malignant melanoma with (18)F-5FPN. European journal of nuclear medicine and molecular imaging 2016, 43, 113-122, doi:10.1007/s00259015-3134-2.

190. Wang, Y.; Li, M.; Zhang, Y.; Zhang, F.; Liu, C.; Song, Y.; Zhang, Y.; Lan, X. Detection of melanoma metastases with PET-Comparison of 18F-5-FPN with 18F-FDG. Nuclear Medicine and Biology 2017, 50, 33-38, doi:https://doi.org/10.1016/j.nucmedbio.2017.03.005.

191. Denoyer, D.; Greguric, I.; Roselt, P.; Neels, O.C.; Aide, N.; Taylor, S.R.; Katsifis, A.; Dorow, D.S.; Hicks, R.J. High-contrast PET of melanoma using (18)F-MEL050, a selective probe for melanin with predominantly renal clearance. Journal of nuclear medicine : official publication, Society of Nuclear Medicine 2010, 51, 441-447, doi:10.2967/jnumed.109.070060.

192. Rizzo-Padoin, N.; Chaussard, M.; Vignal, N.; Kotula, E.; Tsoupko-Sitnikov, V.; Vaz, S.; Hontonnou, F.; Liu, W.Q.; Poyet, J.L.; Vidal, M., et al. [(18)F]MEL050 as a melanin-targeted PET tracer: Fully automated radiosynthesis and comparison to (18)F-FDG for the detection of pigmented melanoma in mice primary subcutaneous tumors and pulmonary metastases. Nucl Med Biol 2016, 43, 773-780, doi:10.1016/j.nucmedbio.2016.08.010.

193. Denoyer, D.; Potdevin, T.; Roselt, P.; Neels, O.C.; Kirby, L.; Greguric, I.; Katsifis, A.; Dorow, D.S.; Hicks, R.J. Improved detection of regional melanoma metastasis using 18F-6-fluoro-N-[2-(diethylamino)ethyl] pyridine3-carboxamide, a melanin-specific PET probe, by perilesional administration. Journal of nuclear medicine : official publication, Society of Nuclear Medicine 2011, 52, 115-122, doi:10.2967/jnumed.110.078154.

194. Xu, X.; Yuan, L.; Yin, L.; Jiang, Y.; Gai, Y.; Liu, Q.; Wang, Y.; Zhang, Y.; Lan, X. Synthesis and Preclinical Evaluation of (18)F-PEG(3)-FPN for the Detection of Metastatic Pigmented Melanoma. Molecular pharmaceutics 2017, 14, 3896-3905, doi:10.1021/acs.molpharmaceut.7b00607.

195. Bashir, U.; Mallia, A.; Stirling, J.; Joemon, J.; MacKewn, J.; Charles-Edwards, G.; Goh, V.; Cook, G.J. PET/MRI in Oncological Imaging: State of the Art. Diagnostics (Basel, Switzerland) 2015, 5, 333-357, doi:10.3390/diagnostics5030333.

196. Nensa, F.; Beiderwellen, K.; Heusch, P.; Wetter, A. Clinical applications of PET/MRI: current status and future perspectives. Diagnostic and interventional radiology (Ankara, Turkey) 2014, 20, 438-447, doi:10.5152/dir.2014.14008.

197. Donati, O.F.; Hany, T.F.; Reiner, C.S.; von Schulthess, G.K.; Marincek, B.; Seifert, B.; Weishaupt, D. Value of retrospective fusion of PET and MR images in detection of hepatic metastases: comparison with 18F-FDG 
PET/CT and Gd-EOB-DTPA-enhanced MRI. Journal of nuclear medicine : official publication, Society of Nuclear Medicine 2010, 51, 692-699, doi:10.2967/jnumed.109.068510.

198. Beiderwellen, K.; Gomez, B.; Buchbender, C.; Hartung, V.; Poeppel, T.D.; Nensa, F.; Kuehl, H.; Bockisch, A.; Lauenstein, T.C. Depiction and characterization of liver lesions in whole body $\left[{ }^{18} \mathrm{~F}\right]-\mathrm{FDG}$ PET/MRI. European journal of radiology 2013, 82, e669-675, doi:10.1016/j.ejrad.2013.07.027.

199. Reiner, C.S.; Stolzmann, P.; Husmann, L.; Burger, I.A.; Hüllner, M.W.; Schaefer, N.G.; Schneider, P.M.; von Schulthess, G.K.; Veit-Haibach, P. Protocol requirements and diagnostic value of PET/MR imaging for liver metastasis detection. European journal of nuclear medicine and molecular imaging 2014, 41, 649-658, doi:10.1007/s00259-013-2654-x.

200. Shokrollahi, H. Contrast agents for MRI. Mater Sci Eng C Mater Biol Appl 2013, 33, 4485-4497, doi:10.1016/j.msec.2013.07.012.

201. Fernandes, P.A.; Carvalho, A.T.; Marques, A.T.; Pereira, A.L.; Madeira, A.P.; Ribeiro, A.S.; Carvalho, A.F.; Ricardo, E.T.; Pinto, F.J.; Santos, H.A., et al. New designs for MRI contrast agents. J Comput Aided Mol Des 2003, 17, 463-473, doi:10.1023/a:1027347527385.

202. Harisinghani, M.G.; Jhaveri, K.S.; Weissleder, R.; Schima, W.; Saini, S.; Hahn, P.F.; Mueller, P.R. MRI contrast agents for evaluating focal hepatic lesions. Clin Radiol 2001, 56, 714-725, doi:10.1053/crad.2001.0764.

203. Rummeny, E.J.; Torres, C.G.; Kurdziel, J.C.; Nilsen, G.; Op de Beeck, B.; Lundby, B. MnDPDP for MR imaging of the liver. Results of an independent image evaluation of the European phase III studies. Acta Radio/ 1997, 38, 638-642.

204. Lin, Y.J.; Koretsky, A.P. Manganese ion enhances T1-weighted MRI during brain activation: an approach to direct imaging of brain function. Magn Reson Med 1997, 38, 378-388, doi:10.1002/mrm.1910380305.

205. Norek, M.; Peters, J.A. MRI contrast agents based on dysprosium or holmium. Prog Nucl Magn Reson Spectrosc 2011, 59, 64-82, doi:10.1016/j.pnmrs.2010.08.002.

206. Holt, K.B. Diamond at the nanoscale: applications of diamond nanoparticles from cellular biomarkers to quantum computing. Philos Trans A Math Phys Eng Sci 2007, 365, 2845-2861, doi:10.1098/rsta.2007.0005.

207. Mornet, S.; Vasseur, S.; Grasset, F.; Veverka, P.; Goglio, G.; Demourgues, A.; Portier, J.; Pollert, E.; Duguet, E. Magnetic nanoparticle design for medical applications. Progress in Solid State Chemistry 2006, 34, 237-247.

208. Lutz, A.M.; Weishaupt, D.; Persohn, E.; Goepfert, K.; Froehlich, J.; Sasse, B.; Gottschalk, J.; Marincek, B.; Kaim, A.H. Imaging of macrophages in soft-tissue infection in rats: relationship between ultrasmall superparamagnetic iron oxide dose and MR signal characteristics. Radiology 2005, 234, 765-775, doi:10.1148/radiol.2343031172.

209. Neuwelt, A.; Sidhu, N.; Hu, C.A.; Mlady, G.; Eberhardt, S.C.; Sillerud, L.O. Iron-based superparamagnetic nanoparticle contrast agents for MRI of infection and inflammation. AJR Am J Roentgenol 2015, 204, W302313, doi:10.2214/ajr.14.12733.

210. Yang, L.; Peng, X.H.; Wang, Y.A.; Wang, X.; Cao, Z.; Ni, C.; Karna, P.; Zhang, X.; Wood, W.C.; Gao, X., et al. Receptor-targeted nanoparticles for in vivo imaging of breast cancer. Clin Cancer Res 2009, 15, 4722-4732, doi:10.1158/1078-0432.Ccr-08-3289.

211. Boles, K.S.; Schmieder, A.H.; Koch, A.W.; Carano, R.A.; Wu, Y.; Caruthers, S.D.; Tong, R.K.; Stawicki, S.; Hu, G.; Scott, M.J., et al. MR angiogenesis imaging with Robo4- vs. alphaVbeta3-targeted nanoparticles in a B16/F10 mouse melanoma model. Faseb j 2010, 24, 4262-4270, doi:10.1096/fj.10-157933.

212. Lacroix, L.M.; Ho, D.; Sun, S. Magnetic nanoparticles as both imaging probes and therapeutic agents. Curr Top Med Chem 2010, 10, 1184-1197, doi:10.2174/156802610791384207. 
213. Taylor, R.M.; Huber, D.L.; Monson, T.C.; Ali, A.M.; Bisoffi, M.; Sillerud, L.O. Multifunctional iron platinum stealth immunomicelles: targeted detection of human prostate cancer cells using both fluorescence and magnetic resonance imaging. J Nanopart Res 2011, 13, 4717-4729, doi:10.1007/s11051-011-0439-3.

214. Matsunaga, T.; Suzuki, T.; Tanaka, M.; Arakaki, A. Molecular analysis of magnetotactic bacteria and development of functional bacterial magnetic particles for nano-biotechnology. Trends Biotechnol 2007, 25, 182-188, doi:10.1016/j.tibtech.2007.02.002.

215. Lin, W.; Pan, Y.; Bazylinski, D.A. Diversity and ecology of and biomineralization by magnetotactic bacteria. Environ Microbiol Rep 2017, 9, 345-356, doi:10.1111/1758-2229.12550.

216. Sun, J.; Li, Y.; Liang, X.J.; Wang, P.C. Bacterial Magnetosome: A Novel Biogenetic Magnetic Targeted Drug Carrier with Potential Multifunctions. J Nanomater 2011, 2011, 469031-469043, doi:10.1155/2011/469031.

217. Felfoul, O.; Mohammadi, M.; Martel, S. Magnetic resonance imaging of Fe3O4 nanoparticles embedded in living magnetotactic bacteria for potential use as carriers for in vivo applications. Annu Int Conf IEEE Eng Med Biol Soc 2007, 2007, 1463-1466, doi:10.1109/iembs.2007.4352576.

218. Boucher, M.; Geffroy, F.; Prévéral, S.; Bellanger, L.; Selingue, E.; Adryanczyk-Perrier, G.; Péan, M.; Lefèvre, C.T.; Pignol, D.; Ginet, N., et al. Genetically tailored magnetosomes used as MRI probe for molecular imaging of brain tumor. Biomaterials 2017, 121, 167-178, doi:10.1016/j.biomaterials.2016.12.013.

219. Zhang, Y.; Ni, Q.; Xu, C.; Wan, B.; Geng, Y.; Zheng, G.; Yang, Z.; Tao, J.; Zhao, Y.; Wen, J., et al. Smart Bacterial Magnetic Nanoparticles for Tumor-Targeting Magnetic Resonance Imaging of HER2-Positive Breast Cancers. ACS Appl Mater Interfaces 2019, 11, 3654-3665, doi:10.1021/acsami.8b15838.

220. Xiang, Z.; Yang, X.; Xu, J.; Lai, W.; Wang, Z.; Hu, Z.; Tian, J.; Geng, L.; Fang, Q. Tumor detection using magnetosome nanoparticles functionalized with a newly screened EGFR/HER2 targeting peptide. Biomaterials 2017, 115, 53-64, doi:10.1016/j.biomaterials.2016.11.022.

221. Lambiase, A.; Mantelli, F.; Bonini, S. Nerve growth factor eye drops to treat glaucoma. Drug news \& perspectives 2010, 23, 361-367, doi:10.1358/dnp.2010.23.6.1472299.

222. Kettunen, M.I. Hyperpolarized MRI for Studying Tumor Metabolism. Methods Mol Biol 2019, 1928, 409-426, doi:10.1007/978-1-4939-9027-6_21.

223. Fiedorowicz, M.; Wieteska, M.; Rylewicz, K.; Kossowski, B.; Piątkowska-Janko, E.; Czarnecka, A.M.; Toczyłowska, B.; Bogorodzki, P. Hyperpolarized $13 \mathrm{C}$ tracers: Technical advancements and perspectives for clinical applications. Biocybernetics and Biomedical Engineering 2021, https://doi.org/10.1016/i.bbe.2021.03.010, doi:https://doi.org/10.1016/i.bbe.2021.03.010.

224. Adamson, E.B.; Ludwig, K.D.; Mummy, D.G.; Fain, S.B. Magnetic resonance imaging with hyperpolarized agents: methods and applications. Phys Med Biol 2017, 62, R81-r123, doi:10.1088/1361-6560/aa6be8.

225. Keshari, K.R.; Wilson, D.M. Chemistry and biochemistry of $13 \mathrm{C}$ hyperpolarized magnetic resonance using dynamic nuclear polarization. Chemical Society reviews 2014, 43, 1627-1659, doi:10.1039/c3cs60124b.

226. Nardi-Schreiber, A.; Gamliel, A.; Harris, T.; Sapir, G.; Sosna, J.; Gomori, J.M.; Katz-Brull, R. Biochemical phosphates observed using hyperpolarized (31)P in physiological aqueous solutions. Nature communications 2017, 8, 341, doi:10.1038/s41467-017-00364-3.

227. Siddiqui, S.; Kadlecek, S.; Pourfathi, M.; Xin, Y.; Mannherz, W.; Hamedani, H.; Drachman, N.; Ruppert, K.; Clapp, J.; Rizi, R. The use of hyperpolarized carbon-13 magnetic resonance for molecular imaging. Advanced drug delivery reviews 2017, 113, 3-23, doi:10.1016/j.addr.2016.08.011.

228. Ji, X.; Bornet, A.; Vuichoud, B.; Milani, J.; Gajan, D.; Rossini, A.J.; Emsley, L.; Bodenhausen, G.; Jannin, S. Transportable hyperpolarized metabolites. Nat Commun 2017, 8, 13975, doi:10.1038/ncomms13975. 
229. Miloushev, V.Z.; Keshari, K.R.; Holodny, A.I. Hyperpolarization MRI: Preclinical Models and Potential Applications in Neuroradiology. Top Magn Reson Imaging 2016, 25, 31-37, doi:10.1097/rmr.0000000000000076.

230. Rajeshkumar, N.V.; Dutta, P.; Yabuuchi, S.; de Wilde, R.F.; Martinez, G.V.; Le, A.; Kamphorst, J.J.; Rabinowitz, J.D.; Jain, S.K.; Hidalgo, M., et al. Therapeutic Targeting of the Warburg Effect in Pancreatic Cancer Relies on an Absence of p53 Function. Cancer Res 2015, 75, 3355-3364, doi:10.1158/0008-5472.Can-15-0108.

231. Sriram, R.; Van Criekinge, M.; Hansen, A.; Wang, Z.J.; Vigneron, D.B.; Wilson, D.M.; Keshari, K.R.; Kurhanewicz, J. Real-time measurement of hyperpolarized lactate production and efflux as a biomarker of tumor aggressiveness in an MR compatible 3D cell culture bioreactor. NMR Biomed 2015, 28, 1141-1149, doi:10.1002/nbm.3354.

232. Schroeder, M.A.; Atherton, H.J.; Dodd, M.S.; Lee, P.; Cochlin, L.E.; Radda, G.K.; Clarke, K.; Tyler, D.J. The cycling of acetyl-coenzyme $A$ through acetylcarnitine buffers cardiac substrate supply: a hyperpolarized $13 C$ magnetic resonance study. Circ Cardiovasc Imaging 2012, 5, 201-209, doi:10.1161/circimaging.111.969451.

233. Felig, P. The glucose-alanine cycle. Metabolism 1973, 22, 179-207, doi:10.1016/0026-0495(73)90269-2.

234. Gallagher, F.A.; Kettunen, M.I.; Hu, D.E.; Jensen, P.R.; Zandt, R.I.; Karlsson, M.; Gisselsson, A.; Nelson, S.K.; Witney, T.H.; Bohndiek, S.E., et al. Production of hyperpolarized [1,4-13C2]malate from [1,4-13C2]fumarate is a marker of cell necrosis and treatment response in tumors. Proc Natl Acad Sci U S A 2009, 106, 1980119806, doi:10.1073/pnas.0911447106.

235. Reed, G.D.; von Morze, C.; Bok, R.; Koelsch, B.L.; Van Criekinge, M.; Smith, K.J.; Hong, S.; Larson, P.E.; Kurhanewicz, J.; Vigneron, D.B. High resolution (13)C MRI with hyperpolarized urea: in vivo T(2) mapping and (15)N labeling effects. IEEE Trans Med Imaging 2014, 33, 362-371, doi:10.1109/tmi.2013.2285120.

236. Ghosh, R.K.; Kadlecek, S.J.; Pourfathi, M.; Rizi, R.R. Efficient production of hyperpolarized bicarbonate by chemical reaction on a DNP precursor to measure pH. Magn Reson Med 2015, 74, 1406-1413, doi:10.1002/mrm.25530.

237. Flavell, R.R.; von Morze, C.; Blecha, J.E.; Korenchan, D.E.; Van Criekinge, M.; Sriram, R.; Gordon, J.W.; Chen, H.Y.; Subramaniam, S.; Bok, R.A., et al. Application of Good's buffers to $\mathrm{pH}$ imaging using hyperpolarized (13)C MRI. Chem Commun (Camb) 2015, 51, 14119-14122, doi:10.1039/c5cc05348j.

238. Jiang, W.; Lumata, L.; Chen, W.; Zhang, S.; Kovacs, Z.; Sherry, A.D.; Khemtong, C. Hyperpolarized 15Npyridine derivatives as pH-sensitive MRI agents. Sci Rep 2015, 5, 9104, doi:10.1038/srep09104.

239. Yen, Y.F.; Kohler, S.J.; Chen, A.P.; Tropp, J.; Bok, R.; Wolber, J.; Albers, M.J.; Gram, K.A.; Zierhut, M.L.; Park, I., et al. Imaging considerations for in vivo $13 \mathrm{C}$ metabolic mapping using hyperpolarized 13C-pyruvate. Magn Reson Med 2009, 62, 1-10, doi:10.1002/mrm.21987.

240. Kurhanewicz, J.; Vigneron, D.B.; Ardenkjaer-Larsen, J.H.; Bankson, J.A.; Brindle, K.; Cunningham, C.H.; Gallagher, F.A.; Keshari, K.R.; Kjaer, A.; Laustsen, C., et al. Hyperpolarized (13)C MRI: Path to Clinical Translation in Oncology. Neoplasia 2019, 21, 1-16, doi:10.1016/j.neo.2018.09.006.

241. Lambiase, A.; Mantelli, F.; Sacchetti, M.; Rossi, S.; Aloe, L.; Bonini, S. Clinical applications of NGF in ocular diseases. Archives italiennes de biologie 2011, 149, 283-292, doi:10.4449/aib.v149i2.1363.

242. Jackson, S.E.; Chester, J.D. Personalised cancer medicine. Int J Cancer 2015, 137, 262-266, doi:10.1002/ijc.28940.

243. Shields, C.L.; Say, E.A.T.; Hasanreisoglu, M.; Saktanasate, J.; Lawson, B.M.; Landy, J.E.; Badami, A.U.; Sivalingam, M.D.; Hauschild, A.J.; House, R.J., et al. Personalized Prognosis of Uveal Melanoma Based on Cytogenetic Profile in 1059 Patients over an 8-Year Period: The 2017 Harry S. Gradle Lecture. Ophthalmology 2017, 124, 1523-1531, doi:10.1016/j.ophtha.2017.04.003. 
244. Bhujwalla, Z.M.; Kakkad, S.; Chen, Z.; Jin, J.; Hapuarachchige, S.; Artemov, D.; Penet, M.F. Theranostics and metabolotheranostics for precision medicine in oncology. J Magn Reson 2018, 291, 141-151, doi:10.1016/j.jmr.2018.03.004.

245. Kim, H.; Kwak, G.; Kim, K.; Yoon, H.Y.; Kwon, I.C. Theranostic designs of biomaterials for precision medicine in cancer therapy. Biomaterials 2019, 213, 119207, doi:10.1016/j.biomaterials.2019.05.018.

246. Dal Corso, A.; Pignataro, L.; Belvisi, L.; Gennari, C. $\alpha$ v 33 Integrin-Targeted Peptide/Peptidomimetic-Drug Conjugates: In-Depth Analysis of the Linker Technology. Curr Top Med Chem 2016, 16, 314-329, doi:10.2174/1568026615666150701114343.

247. Kobayashi, H.; Choyke, P.L. Target-cancer-cell-specific activatable fluorescence imaging probes: rational design and in vivo applications. Acc Chem Res 2011, 44, 83-90, doi:10.1021/ar1000633.

248. Sun, W.; Li, M.; Fan, J.; Peng, X. Activity-Based Sensing and Theranostic Probes Based on Photoinduced Electron Transfer. Acc Chem Res 2019, 52, 2818-2831, doi:10.1021/acs.accounts.9b00340.

249. Ling, D.; Park, W.; Park, S.J.; Lu, Y.; Kim, K.S.; Hackett, M.J.; Kim, B.H.; Yim, H.; Jeon, Y.S.; Na, K., et al. Multifunctional tumor $\mathrm{pH}$-sensitive self-assembled nanoparticles for bimodal imaging and treatment of resistant heterogeneous tumors. J Am Chem Soc 2014, 136, 5647-5655, doi:10.1021/ja4108287.

250. Kim, K.S.; Kim, J.; Lee, J.Y.; Matsuda, S.; Hideshima, S.; Mori, Y.; Osaka, T.; Na, K. Stimuli-responsive magnetic nanoparticles for tumor-targeted bimodal imaging and photodynamic/hyperthermia combination therapy. Nanoscale 2016, 8, 11625-11634, doi:10.1039/c6nr02273a.

251. de la Rica, R.; Aili, D.; Stevens, M.M. Enzyme-responsive nanoparticles for drug release and diagnostics. Adv Drug Deliv Rev 2012, 64, 967-978, doi:10.1016/j.addr.2012.01.002.

252. Kim, E.J.; Bhuniya, S.; Lee, H.; Kim, H.M.; Cheong, C.; Maiti, S.; Hong, K.S.; Kim, J.S. An activatable prodrug for the treatment of metastatic tumors. J Am Chem Soc 2014, 136, 13888-13894, doi:10.1021/ja5077684.

253. Zhou, Y.; Maiti, M.; Sharma, A.; Won, M.; Yu, L.; Miao, L.X.; Shin, J.; Podder, A.; Bobba, K.N.; Han, J., et al. Azo-based small molecular hypoxia responsive theranostic for tumor-specific imaging and therapy. J Control Release 2018, 288, 14-22, doi:10.1016/j.jconrel.2018.08.036.

254. Langbein, T.; Weber, W.A.; Eiber, M. Future of Theranostics: An Outlook on Precision Oncology in Nuclear Medicine. J Nucl Med 2019, 60, 13s-19s, doi:10.2967/jnumed.118.220566.

255. Lecocq, Q.; De Vlaeminck, Y.; Hanssens, H.; D'Huyvetter, M.; Raes, G.; Goyvaerts, C.; Keyaerts, M.; Devoogdt, N.; Breckpot, K. Theranostics in immuno-oncology using nanobody derivatives. Theranostics 2019, 9, $7772-$ 7791, doi:10.7150/thno.34941.

256. Niederkorn, J.Y. Ocular immune privilege and ocular melanoma: parallel universes or immunological plagiarism? Front Immunol 2012, 3, 148, doi:10.3389/fimmu.2012.00148.

257. Yu, X.; Huang, X.; Chen, X.; Liu, J.; Wu, C.; Pu, Q.; Wang, Y.; Kang, X.; Zhou, L. Characterization of a novel antihuman lymphocyte activation gene 3 (LAG-3) antibody for cancer immunotherapy. MAbs 2019, 11, 11391148, doi:10.1080/19420862.2019.1629239.

258. Pereira, P.R.; Odashiro, A.N.; Lim, L.A.; Miyamoto, C.; Blanco, P.L.; Odashiro, M.; Maloney, S.; De Souza, D.F.; Burnier, M.N., Jr. Current and emerging treatment options for uveal melanoma. Clin Ophthalmol 2013, 7, 1669-1682, doi:10.2147/opth.S28863.

259. Boudousquie, C.; Bossi, G.; Hurst, J.M.; Rygiel, K.A.; Jakobsen, B.K.; Hassan, N.J. Polyfunctional response by ImmTAC (IMCgp100) redirected CD8(+) and CD4(+) T cells. Immunology 2017, 152, 425-438, doi:10.1111/imm.12779. 
